Med. Hist. (2015), vol. 59(2), pp. 275-326. C The Author 2015. Published by Cambridge University Press 2015 The online version of this article is published within an Open Access environment subject to the conditions of the Creative Commons Attribution licence $<\mathrm{http} / / /$ creativecommons.org/licenses/by/3.0/ $>$.

doi: $10.1017 / \mathrm{mdh} .2015 .6$

\title{
Greek Manuscripts at the Wellcome Library in London: A Descriptive Catalogue
}

\author{
PETROS BOURAS-VALLIANATOS* \\ Centre for Hellenic Studies, King's College London, Strand, \\ WC2R 2LS, London, UK \\ with contributions by GEORGI R. PARPULOV \\ Department of Britain, Prehistory and Europe, British Museum, \\ Great Russell St, WC1B 3DG, London, UK
}

\begin{abstract}
This article presents a new, detailed catalogue of the Greek manuscripts at the Wellcome Library in London. It consists of an introduction to the history of the collection and its scholarly importance, followed by separate entries for each manuscript. Each entry identifies the text(s) found in the respective manuscript - including reference to existing printed edition(s) of such texts - and gives a physical description of the codex, details on its provenance and bibliographical references.
\end{abstract}

Keywords: Greek/Byzantine medicine, Greek/Byzantine medical manuscripts, Greek palaeography/codicology, Wellcome Library, Medical Society of London

\footnotetext{
* Email address for correspondence: petros.bouras-vallianatos@ hotmail.com

I would like to express my gratitude to Georgi Parpulov, who first aroused my interest in Greek palaeography and codicology; his continuous encouragement and numerous contributions have been extremely useful in preparing this catalogue. I am further indebted to the anonymous referees for their constructive comments on earlier drafts of this paper, and to the editor, Sanjoy Bhattacharya, for his promptness in our correspondence and his care in publishing this article. Special thanks go to the Head of the Wellcome Library, Simon Chaplin, and the Head of Research and Special Collections, Richard Aspin, for their continuous support throughout this Wellcome Library funded project (D237). I am also grateful to Elma Brenner (Medieval and Early Modern Medicine Specialist), Chris Hilton (Senior Archivist), Nikolai Serikoff (Asian Collections Librarian) and all the Wellcome librarians for facilitating access to their Special Collections. I would like to thank Robert Ireland for allowing access to the Library of the Medical Society of London, where I had the opportunity to consult old annotated printed catalogues. I am also thankful to Ludmilla Jordanova for her significant support during the early stages of this project. Furthermore, I have benefited from comments and bibliographical references on particular manuscripts by Irene Calà, Roberta Giubilini, Marjolijne Janssen, Gabrielle Lherminier, Rebekah Lunt, Rudolf Stefec, Anne Tihon, Sophia Xenophontos, and Barbara Zipser. Many thanks go to the Wellcome Library Open Access Fund for covering the open access publishing costs. The Library has begun digitising its entire collection of pre-Western European manuscripts, including all Greek manuscripts, which will be available on the library's website soon (http://blog.wellcomelibrary.org/2014/05/digitising-the-librarys-medieval-manuscripts/, accessed 18 December 2014).
} 


\section{Introduction}

The Wellcome Library currently owns sixteen Greek manuscripts. ${ }^{1}$ These can be divided into two main groups according to their provenance and date of purchase. The first group consists of five volumes (MSS 289, 354, 413, 498 and 4103), all acquired separately between 1910 and 1936, while Sir Henry Wellcome (1853-1936) was still alive. The second group forms the core of the collection and is made up of eleven codices, previously owned by the Medical Society of London [MSS M(edical)S(ociety)L(ondon) 1, 14, 52, 60, $62,109,112,114,124,126$ and 135]. ${ }^{2}$ These were part of the library's largest acquisition since Sir Henry Wellcome's death, viz., about 10000 books and 200 manuscripts initially transferred on long-term loan to the Wellcome Library in 1967 thanks to the efforts of its director Noel Poynter (1906-79), and finally purchased in 1984.

Wellcome MSS MSL 1, 14, 52, 60, 62, 109 and 114 carry uniform bindings of gilttooled brown leather over wooden boards, with marbled tail edges and endpapers. (All their spines have been rebacked, evidently in the late nineteenth century.) Two tools used for their corner-piece ornament are identical to those on an unsigned mid-eighteenth-century binding $^{3}$ and to some employed by the London bookbinder and publisher John Brindley (1692?-1758). ${ }^{4}$ MSS 52, 62, 109 and 114 are marked as having been the property of the physician and bibliophile Anthony Askew (1722-74), ${ }^{5}$ other manuscripts from whose collection have very similar bindings. ${ }^{6}$ In the 1785 sale catalogue of Askew's library such bindings are referred to as 'in Russian leather' (corio russico). ${ }^{7}$ (For brevity's sake, I have used the same designation in my catalogue.) Except for MS.MSL.135, these manuscripts can be identified in the 1785 sale catalogue. Askew is known to have bought from the physician, collector and philanthropist Richard Mead (1673-1754) all the Greek medical manuscripts that the latter had collected. ${ }^{8}$ Since no record of Mead's acquisitions survives,

${ }^{1}$ In addition to the Greek manuscripts, the library holds a few Greek papyri fragments (MSS 5753, 5754 and 5755); see, Vivian Nutton, 'Wellcome papiro Oxyrhynchus 2547 e papiro Johnson', Kos, 1, 6 (1984), 7-9, and Richard Palmer, Catalogue of Western manuscripts in the Library of the Wellcome Institute for the History and Understanding of Medicine: western manuscripts 5120-6244 (London: The Wellcome Trust, 2000), 142-4. For a history of the Wellcome Library, see John Symons, Wellcome Institute for the History of Medicine. A Short History (London: The Wellcome Trust, 1993).

2 On the library of the Medical Society of London, see Penelope Hunting, The Medical Society of London, 1773-2003 (London: Medical Society of London, 2003), 21-6, 291-305.

${ }^{3}$ British Library, shelfmark 195a20: British Library Database of Bookbindings (http://www.bl.uk/catalogues/bo okbindings, accessed 18 December 2014). Cf. Wellcome MS.MSL.62.

${ }^{4}$ British Library, shelfmarks c46g7 and 269i10: ibid. Cf. Wellcome MS.MSL.52A and B. On John Brindley: George Smith, The Oldest London Bookshop: A History of Two Hundred Years (London: Ellis, 1928), 3-20.

5 Anthony Askew studied at Cambridge and Leiden, and was appointed physician to St Bartholomew's Hospital in 1754. His surviving correspondence with Richard Mead and the German philologist Johann Jakob Reiske (1716-74) reflects his great love of Greek literature, including a project for a critical edition of Aeschylus' works, which was never realised. On Askew and his library, see William Younger Fletcher, English Book Collectors (London: Kegan Paul, Trench, Trübner \& Co., 1902), 219-21; and Seymour de Ricci, English Collectors of Books \& Manuscripts (1530-1930) and Their Marks of Ownership (Cambridge: Cambridge University Press, 1930), 47, 52-3.

${ }^{6}$ Eg. British Library, Add. MSS 5108 and 5119 (http://www.bl.uk/manuscripts, accessed 18 December 2014). The latter binding is identical to that of Wellcome MS.MSL.14.

${ }^{7}$ Bibliotheca Askeviana manu scripta, sive catalogus librorum manuscriptorum Antonii Askew (London: Leigh \& Sotheby, 1785), passim.

8 [William Macmichael], The Gold-Headed Cane, 4th edn (New York: Hoever, 1915), 151-2. Richard Mead studied at Utrecht, Leiden and Padua, and was elected physician to St Thomas' Hospital in Southwark in 1703. He wrote several works on medical topics, including an influential monograph on the practice of inoculation for smallpox. He knew Greek and Latin, and his vast collection of books and manuscripts numbered some 10000 volumes. 
it is impossible to ascertain how many - and which - of Askew's numerous codices came from him. Those now owned by the Wellcome were purchased at the above-mentioned 1785 sale by the Anglo-Irish physician James Sims (1741-1820), who sold them to the Medical Society of London in $1802 .^{9}$

The majority of the manuscripts, thirteen in number, contain medical texts. These range in date from treatises of the Hippocratic Corpus to post-Byzantine works, but the bulk is medieval. MSS MSL 14, 60 and MS.4103 preserve various collections of Byzantine and post-Byzantine iatrosophia, an (as yet poorly studied) type of physician's handbook consisting of simple recipes for use in daily practice. ${ }^{10}$ MS.MSL.60, in particular, contains a variety of medical compilations with either a therapeutic or diagnostic focus, eg. uroscopy, and shows how medical texts were adapted in various versions, according to the needs of Byzantine practitioners. The author best represented in the Wellcome collection (MSS MSL 52, 112 and 124) is the late Byzantine physician John Zacharias Aktouarios (ca. 1275-ca. 1330), whose work Medical Epitome has not been fully edited in print yet. Three manuscripts (MSS MSL 62, 109 and 114) with works by Aretaeus (ca. first century AD) and Rufus of Ephesus (ca. AD 80-ca. AD 150), Aetios of Amida (ca. first half of the sixth century AD) and Paul of Aegina (late sixth century AD-d. post 642), respectively, have so far been overlooked by modern scholars. The same applies to MS.MSL.135, whose text of Theophanes Chrysobalantes' (ca. tenth century AD) Medical Epitome was not considered by Joseph Sonderkamp in his detailed study of the textual tradition of that work. ${ }^{11}$ The same codex also contains a previously uncatalogued abridged version of Symeon Seth's (ca. second half of the eleventh century AD) On the Capacities of Foodstuffs.

The non-medical manuscripts include a seventeenth-century mathematarion (MS.MSL.1), ie., an advanced textbook used by Greek students during the period of Ottoman rule (Tourkokratia). ${ }^{12}$ MS.413 is an illustrated collection of Byzantine oracles. Lastly, MS.498 presents late antique and Byzantine astronomical texts, together with some autograph religious poems from the late fifteenth century.

The Greek manuscripts of the London Medical Society have been described three times: by Charles Victor Daremberg (1817-72), by Joseph Baldwin Nias (1856-1919) and by Warren Royal Dawson (1888-1968). Those manuscripts acquired by the Wellcome Library separately between 1910 and 1936 were catalogued by its librarian Samuel Arthur Joseph Moorat (1892-1975). The majority are described below in full detail. MSS MSL 112, 124 and 126, however, have only been given summary descriptions, since they are relatively recent copies, made in England, of Greek texts found in earlier codices. I hope to assist future scholarly research by providing descriptions that are more detailed than the ones heretofore available.

\footnotetext{
9 James Sims studied at Leiden and served as a physician to the General Dispensary in Aldersgate Street and to the Surrey Dispensary. He was President of the Medical Society of London for twenty-two years (1786-1808).

10 On Byzantine iatrosophia, see Anna Maria Ieraci Bio, 'Testi medici di uso strumentale', Jahrbuch der Österreichischen Byzantinistik, 32 (1982), 33-43. For an overview of post-Byzantine collections of recipes,

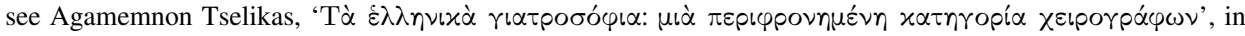

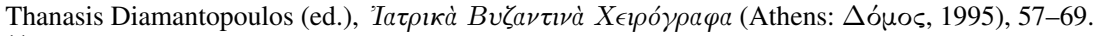

11 Joseph Sonderkamp, Untersuchungen zur Überlieferung der Schriften des Theophanes Chrysobalantes sog. Theophanes Nonnos (Bonn: Habelt, 1987). A critical edition of the text is currently being prepared by Barbara Zipser.

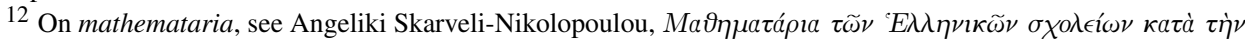

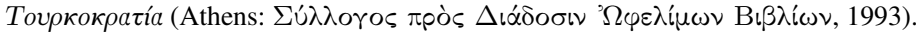




\section{Note on Contents and Bibliography}

The heading of each catalogue entry is organised as follows: [current and former shelfmarks of the manuscript], [its summary contents], [place of origin], [date], [writing material], [number of folia, ie. leaves], [height and width of leaves], [number of lines per page], [justification, ie. height and width of the written area], [ruling type]. All measurements are given in millimetres. Types of ruling are identified according to Jacques-Hubert Sautel (ed.), Répertoire de réglures dans les manuscrits grecs sur parchemin (Turnhout: Brepols, 1995) [Bibliologia, 13]. The ruling is always, unless otherwise specified, in drypoint. The location of quire signatures is described as follows: $S$ (upra) - top of the page, $I$ (nfra) - bottom of the page; $i$ - internal page corner, $m-$ mid-margin, $e$ - external page corner; 1 - first page of the quire, 2 - first and last pages of the quire, 3 - last page of the quire. Titles are given in bold; inc(ipit) and des(init) refer respectively to the opening and ending phrase of a certain work or of a section of a certain work. As a large number of the medical texts do not have a critical edition, in addition to beginning and ending I give a detailed account of the table of contents (where available), because this may be useful for determining the place of a codex within the tradition of a given text. Transcriptions from Greek are diplomatic and retain the spelling and punctuation of the relevant codex. In those cases when a printed edition corresponds to a text found in one of the catalogued manuscript, the name of its editor is given in \{brackets\}, followed by the corresponding page and/or line numbers. (If the printed text differs somewhat from the one found in the MS, its editor's name is preceded by the sign $\sim$.) The full title of the edition can be found either in a footnote or in the bibliography at the end of the corresponding catalogue entry. If a text is included in the TLG database, I provide the relevant reference; the TLG version does not necessarily correspond to the version of the text as it stands in a certain manuscript. For each work, I provide the most common English title. Names of ancient and Byzantine authors follow $O C D$ and $O D B$, respectively. I refer to texts in the Hippocratic Corpus as being by [Hippocrates]. I have employed the following bibliographical abbreviations:

Bibl. Askev. Bibliotheca Askeviana manu scripta, sive catalogus librorum manuscriptorum Antonii Askew (London: Leigh \& Sotheby, 1785)

Cat. Med. Soc. 1803 A Catalogue of Books Contained in the Library of the Medical Society of London, Instituted AD 1773 (London: Medical Society of London, 1803)

Cat. Med. Soc. 1829 A Catalogue of Books Contained in the Library of the Medical Society of London, Instituted AD 1773 (London: Medical Society of London, 1829)

CMG Corpus Medicorum Graecorum (http://cmg.bbaw.de, accessed 18 December 2014)

Daremberg Charles Victor Daremberg, Notices et extraits des manuscrits médicaux grecs, latins et français des principales bibliothèques de l'Europe (Paris: J.-B.Baillière, 1853)

Dawson Warren Royal Dawson, Manuscripta Medica: A Descriptive Catalogue of the Manuscripts in the Library of the Medical Society of London (London: J. Bale, Sons \& Danielsson, 1932) 
Diels Hermann Diels, Die Handschriften der antiken Ärzte, 3 vols (Berlin, 1905-8) [Philosophische und historische Abhandlungen der Königlich Preussischen Akademie der Wissenschaften, (1905) 3, (1906) 1, (1908)]

Moorat Samuel Arthur Joseph Moorat, Catalogue of Western Manuscripts on Medicine and Science in the Wellcome Historical Medical Library, 3 vols (London: Wellcome Institute, 1962-73)

Nias Joseph Baldwin Nias, 'Special Report on the Greek MSS in the Society's Library', Transactions of the Medical Society of London, 27 (1905), lii-lvii

Nutton Vivian Nutton, 'The Legacy of Hippocrates: Greek Medicine in the Library of the Medical Society of London', Transactions of the Medical Society of London, 103 (1986-7), 21-30

Nutton and Zipser Vivian Nutton and Barbara Zipser, 'A Wellcome Manuscript of a Medical Practitioner', in Véronique Boudon-Millot et al. (eds), Storia della tradizione e edizione dei medici greci: Atti del VI Colloquio Internazionale, Paris 12-14 Aprile 2008 (Naples: D’Auria, 2010), 259-70

OCD Simon Hornblower, Anthony Spawforth and Esther Eidinow (eds), The Oxford Classical Dictionary, 4th edn (Oxford: Oxford University Press, 2012)

ODB Alexander Kazhdan (ed.), The Oxford Dictionary of Byzantium, 3 vols (Oxford: Oxford University Press, 1991)

Piccard Wasserzeichenkartei Piccard (http://www.piccard-online.de, accessed 18 December 2014)

RGK Herbert Hunger (ed.), Repertorium der griechischen Kopisten, 800-1600, 3 vols in 9 pts. (Vienna: Verlag der Österreichischen Akademie der Wissenschaften, 1981-97) [cited with volume no. followed by catalogue no., eg. RGKI 213]

TLG Thesaurus Linguae Graecae (http://www.tlg.uci.edu, accessed 18 December 2014)

Touwaide Alain Touwaide, 'Byzantine Medical Manuscripts: Towards a New Catalogue, with a Specimen for an Annotated Checklist of Manuscripts Based on an Index of Diels' Catalogue', Byzantion, 79 (2009), 453-595

\section{MS.MSL.1 (olim HH i 16 / We 15)}

Ottoman Empire, ca. 1620-1640 AD (from watermarks).

Paper, $207 \times$ 147, iii $+199+$ iv (foliated 1 [flyleaf iii]-200), linn. 22 [ca. $157 \times 100$ ], unruled.

Mathematarion: [2r-163v] Anonymous commentary on Aristotle's Categories. ${ }^{13}$ [166r-174r] Anonymous collection of brief theological texts. [175r-200v] Anonymous commentary on Aristotle's Prior and Posterior Analytics.

13 Occasionally the text presents similarities with Porphyry's commentary on Aristotle's Categories; cf. Adolf Busse, Porphyrii Isagoge et in Aristotelis Categorias commentarium (Berlin: Reimer, 1887). [Commentaria in Aristotelem Graeca, Vol. 4.1], 55-142. 


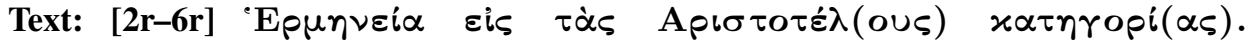

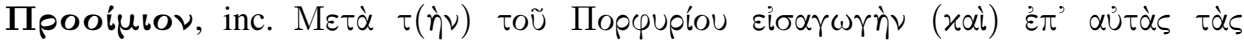

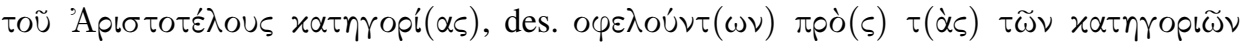

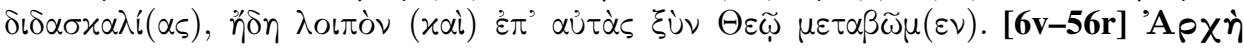

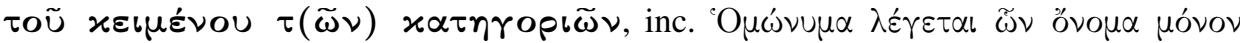

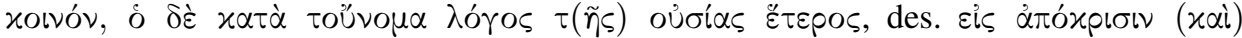

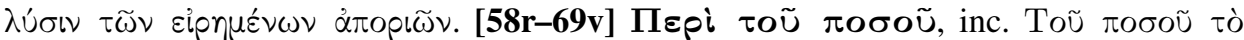

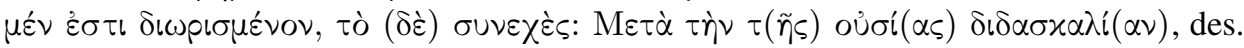

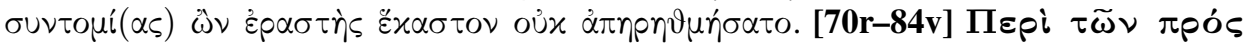

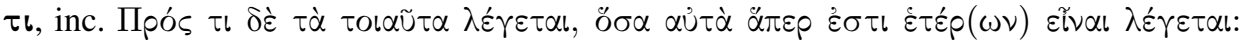

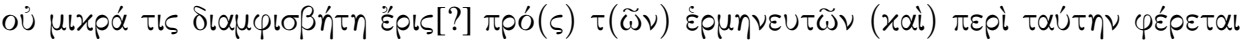

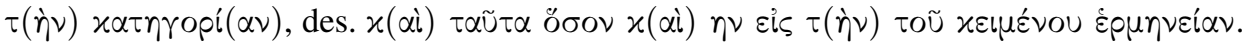

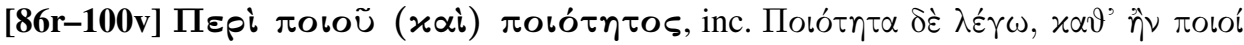

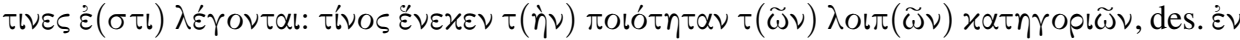

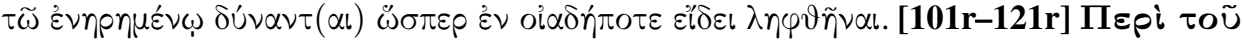

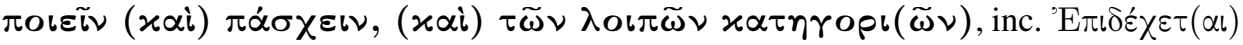

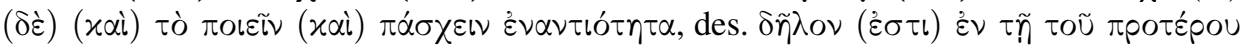

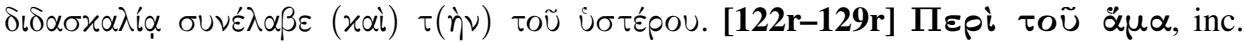

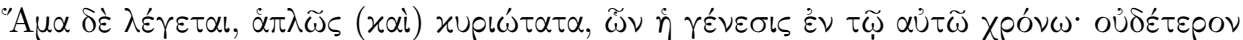

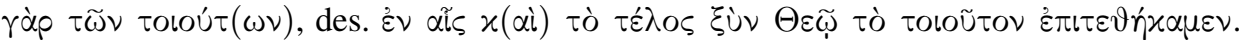

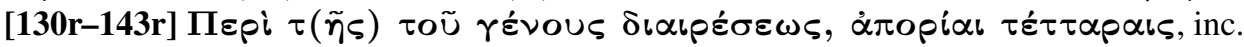

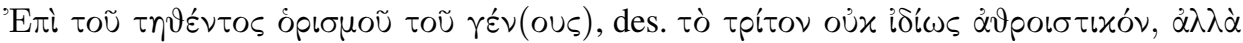

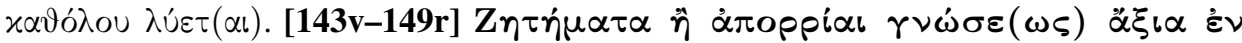

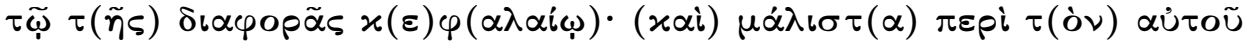

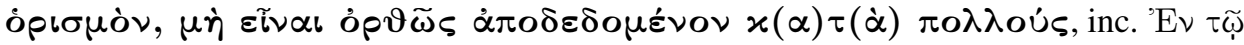

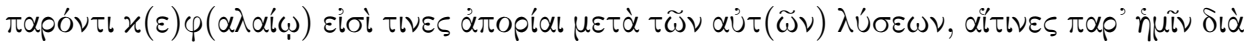

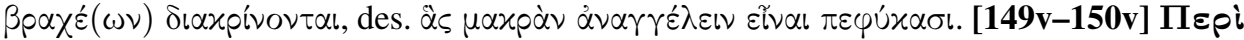

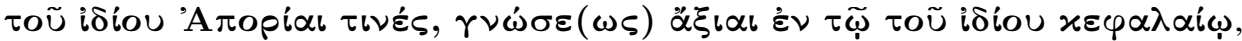

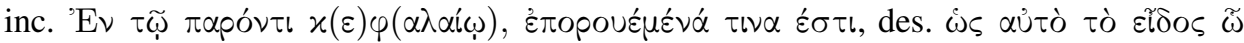

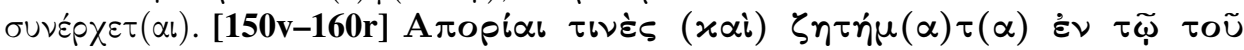

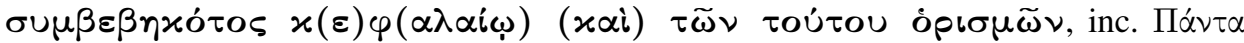

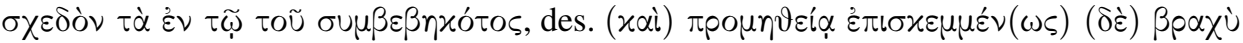

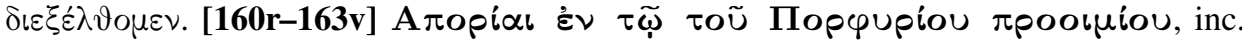

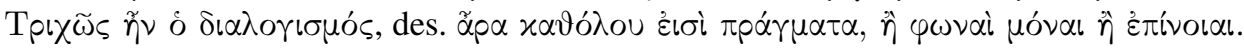

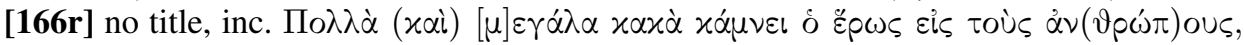

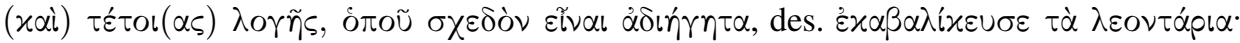

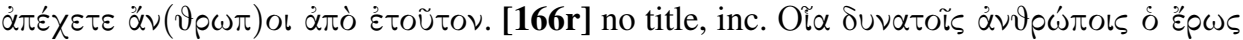

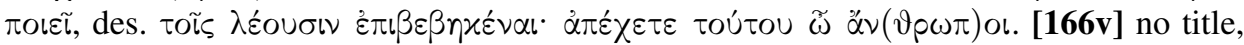

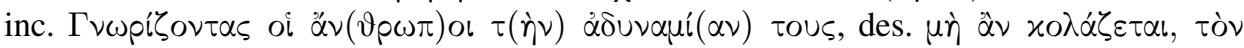

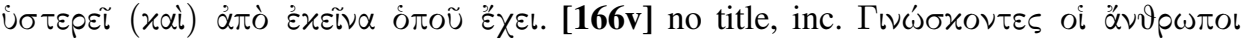

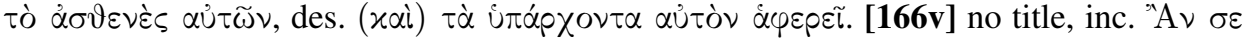

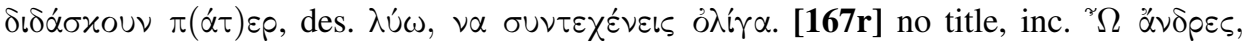

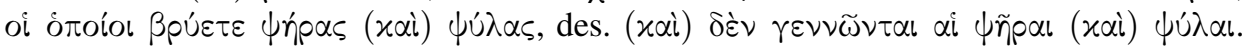

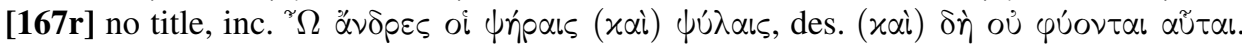

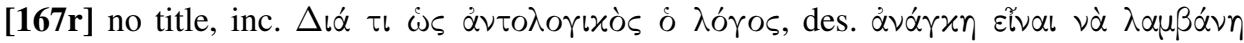

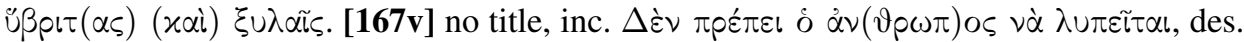

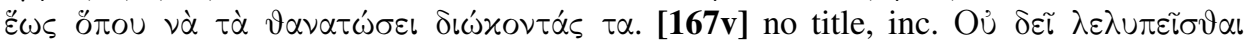




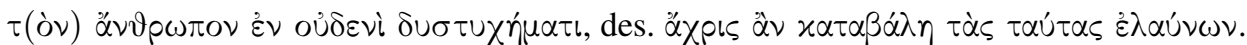

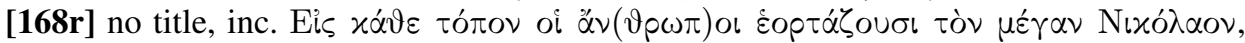

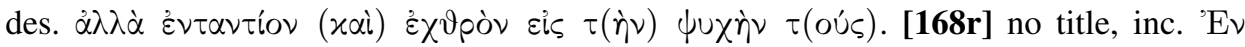

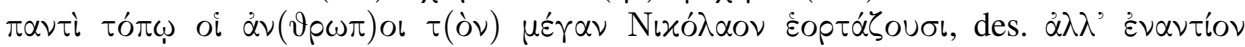

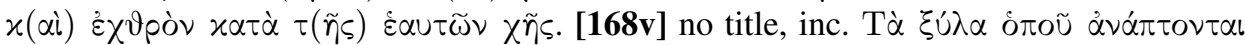

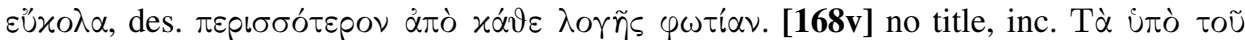

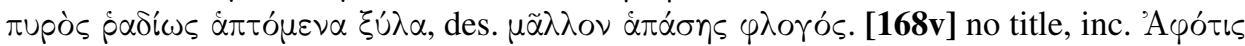

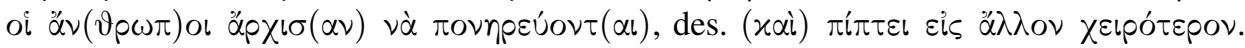

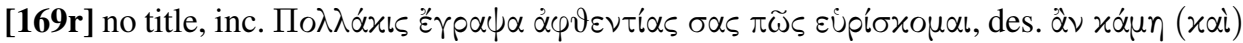

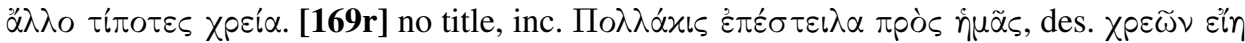

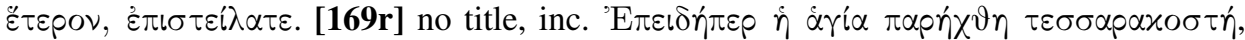

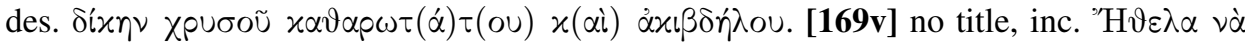

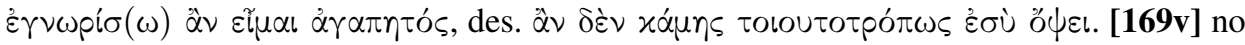

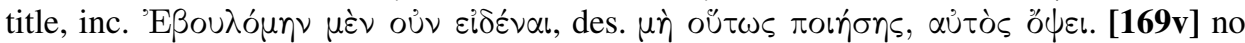

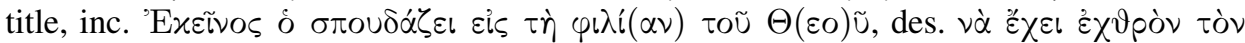

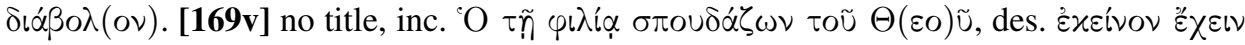

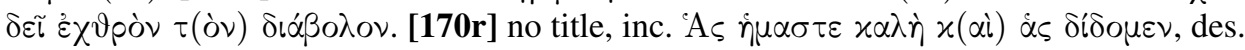

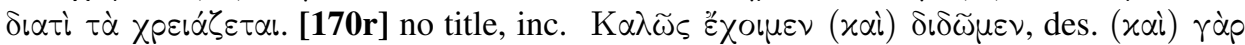

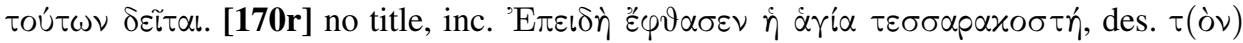

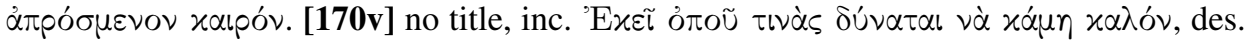

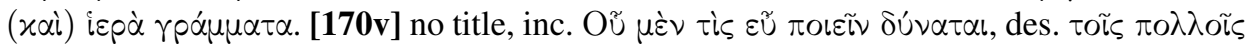

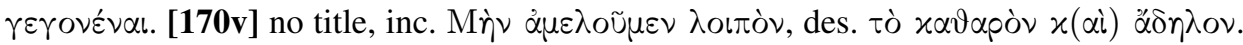

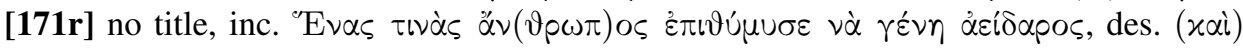

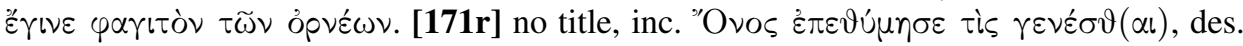

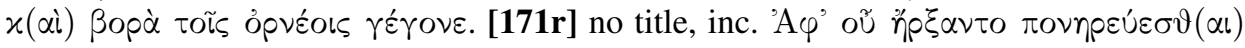

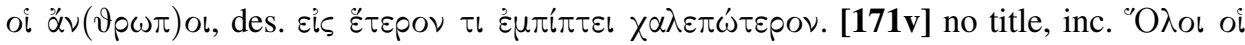

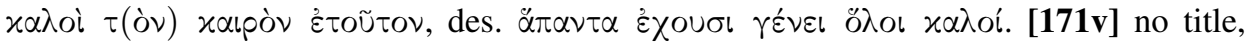

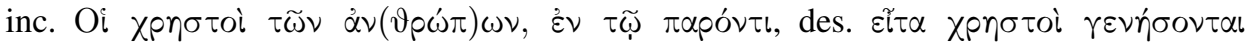

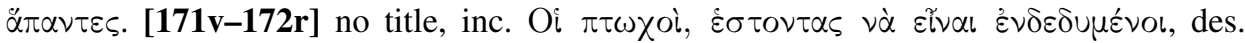

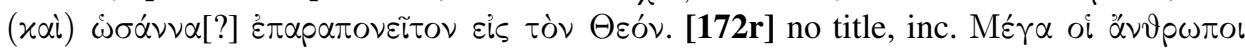

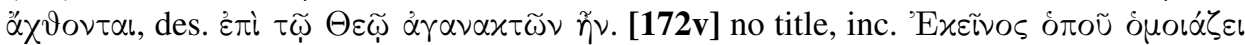

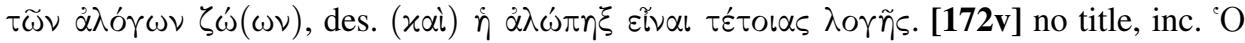

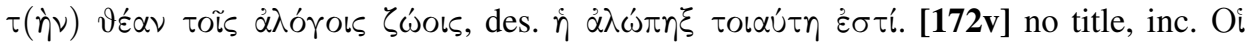

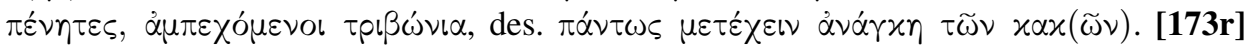

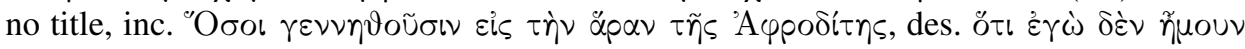

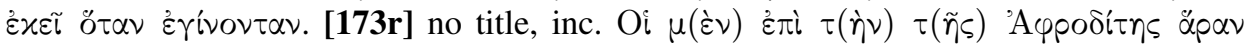

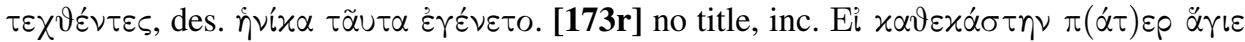

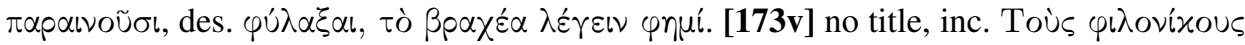

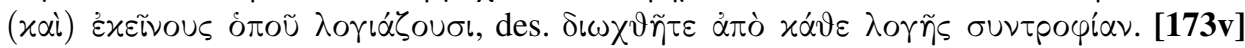

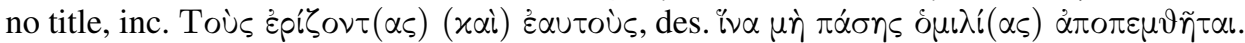

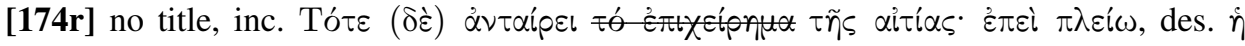

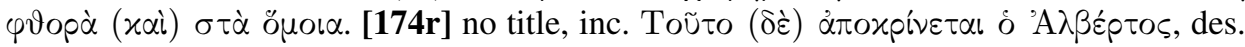

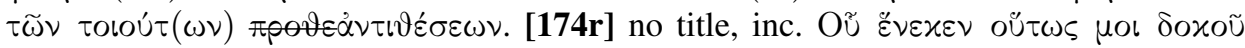

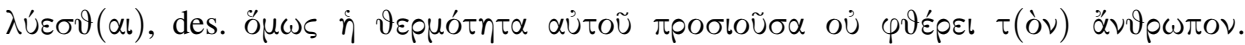

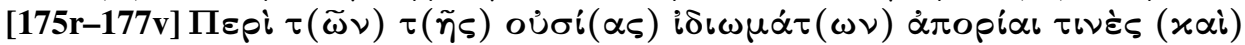

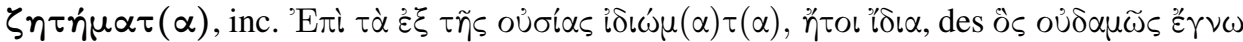

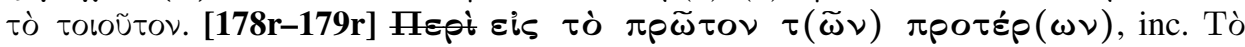




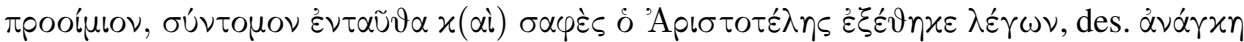

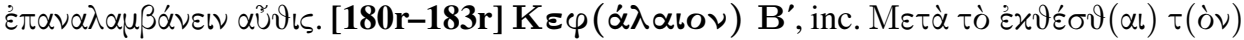

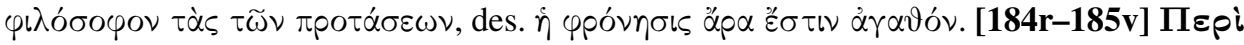

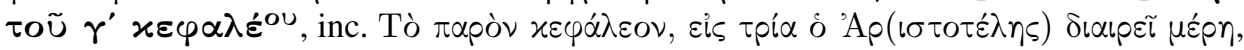

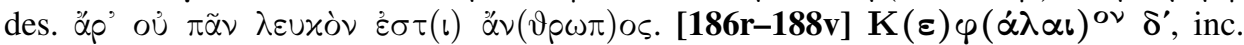

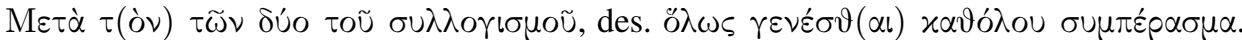

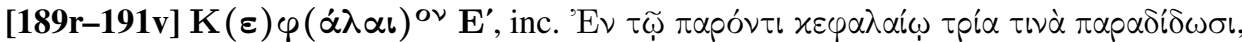

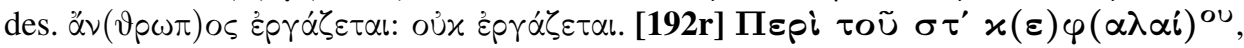

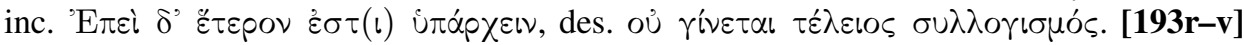

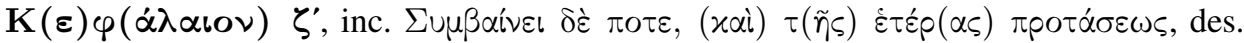

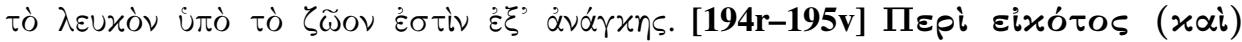

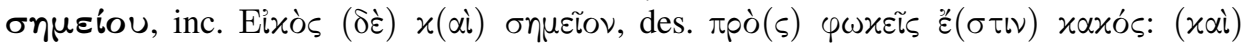

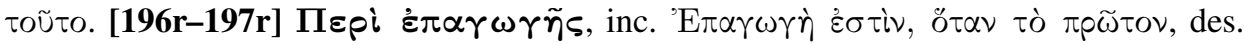

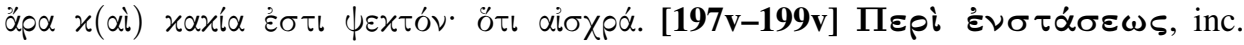

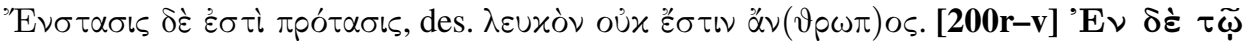

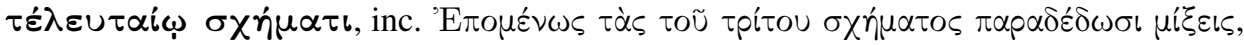

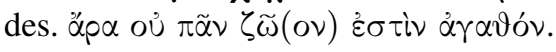

Note: $(194 \mathrm{v}, 197 \mathrm{r})$ the text is written upside down.

Blank pages: $56 \mathrm{v}-57 \mathrm{v}, 85 \mathrm{r}-\mathrm{v}, 121 \mathrm{v}, 129 \mathrm{v}, 164 \mathrm{r}-165 \mathrm{v}, 174 \mathrm{v}, 179 \mathrm{v}, 183 \mathrm{v}, 192 \mathrm{v}$.

Annotations (scribal): (passim) text occasionally crossed out and sometimes corrected (2r, 15r, 22r, 24r, 26r, 27v, 52r, 56r, 58r, 59r, 70r, 73v, 77v, 82v, 89v, 92r, 100r, 113r, 117v, $118 \mathrm{r}, 121 \mathrm{r}, 132 \mathrm{v}, 133 \mathrm{v}, 140 \mathrm{r}, 140 \mathrm{v}, 142 \mathrm{v}, 143 \mathrm{v}, 144 \mathrm{v}, 146 \mathrm{r}, 146 \mathrm{v}, 147 \mathrm{r}, 149 \mathrm{r}, 151 \mathrm{v}, 153 \mathrm{r}$, $159 \mathrm{r}, 160 \mathrm{r}, 166 \mathrm{r}, 169 \mathrm{r}, 170 \mathrm{r}, 173 \mathrm{v}, 175 \mathrm{r})$ text expanded or glossed with synonyms in the

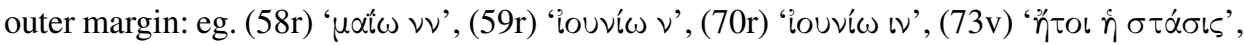
(108v) 'öpos $\sigma \tau \varepsilon p \eta ́ \sigma \varepsilon(\omega \varsigma) /$ definitio privationis' - (29r, 127r, 181r-200r) explanatory diagrams in the outer margin.

Handwriting: unidentified post-Byzantine hand.

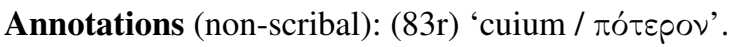

Paper: folded in $4^{\circ}$; chain distance $27 \mathrm{~mm}$; watermarks: (ff. 2-165, 186-200) similar to Velkov Arbalète 20-21, attested, respectively, in 1630 and 1635 - (ff. 166-173) similar (excl. countermark) to Heawood 4 (anchor), attested in 1620 - (ff. 174-185) similar to Heawood 951 (cross), attested in $1618 .^{14}$

Quire signatures (scribal): Greek numerals $\operatorname{Im} 3 \alpha$ (185v), $\operatorname{Im} 2 \beta$ (186r, 193v); Arab numerals $I e$ in the middle of each quire, from $9(5 \mathrm{v}-6 \mathrm{r})$ through $25(127 \mathrm{v}-128 \mathrm{r})$.

Quires: 1 (flyleaf iii), 2 (f. 2), 4 (f. 6), $6 \times 8$ (f. 53), 4 (f. 57), $12 \times 8$ (f. 161), 4 (f. 165), 8 (f. 173), 4 (f. 177), $2 \times 8$ (f. 193), 7 (f. 200), 3 (back flyleaves i-iii).

Binding: corio russico, same tooling as Wellcome MS.MSL.60. - Flyleaves i-1 and 201203: chain distance $25 \mathrm{~mm}$, no watermarks.

Marks of ownership: (front flyleaf II $v$ ) 'H H i 16' - (1r) 'Varii in Aristotelem' - (back pastedown) label inscribed 'L.g.11'.

14 Asparukh Velkov, Les filigranes dans les documents ottomans: divers types d'images (Sofia: Trayanov, 2005); Edward Heawood, Watermarks, Mainly of the 17th and 18th Centuries (Hilversum: Paper Publications Society, 1950). 
Provenance: Anthony Askew (1722-74), London; [his sale, G. Leigh and J. Sotheby, London, 16 March 1785, lot 604]; purchased by James Sims (1741-1820), London, in 1785; purchased by the London Medical Society in 1802; purchased by the Wellcome Library in 1984.

Bibliography: Bibl. Askev., 39 (no. 604); Cat. Med. Soc. 1803, 210; Cat. Med. Soc. 1829, 323; Nias, liv; Dawson, 7.

Roxane D. Argyropoulos and Iannis Caras, Inventaire des manuscrits grecs d'Aristote et de ses commentateurs: contribution à l'histoire du texte d'Aristote. Supplément (Paris: Les Belles Lettres, 1980), 33 (no. 214).

\section{MS.MSL.14 (olim AA a 1 / Xa 32)}

Possibly Cyprus (according to Barbara Zipser and Agamemnon Tselikas), fourteenth century.

Paper, $143 \times 103$, iii + 157 + iii (paginated 1-71, 73-131, 131-189, 191-232, 234-257, 259-317).

[1-12] Ps.-Hippocrates, Epistle to King Ptolemy on the Constitution of Man (TLG 0751.002). [12-14] Alexander the Sophist or Ps.-Hippocrates, On the Human Body and Birth. [14-16] Anonymous opuscule on phlebotomy. ${ }^{15}$ [17-18] Anonymous opuscule on conception. ${ }^{16}$ [18-34] Anonymous collection of remedies. ${ }^{17}$ [34-41] Ps.Hippocrates, Sayings about Life and Death. [41-44] Anonymous collection of remedies. ${ }^{18}$ [44-46] Ps.-Esdras, On Illuminating Days. ${ }^{19}$ [46-76] Anonymous collection of recipes on composite drugs. [76-81] Excerpt from an abridged edition of the vernacular version of John Archiatros' iatrosophion. [84-107] Anonymous collection of remedies. ${ }^{20}$ [82-83, 108-271] Incomplete vernacular version of John Archiatros' iatrosophion. ${ }^{21}$ [272-317] Anonymous medical compilation. ${ }^{22}$

Note: This MS consists of two distinct parts, described below separately as A and $\mathbf{B}$.

\footnotetext{
15 The first few phrases of the text coincide with the last paragraph (VI) of the treatise On Human Birth attributed to Alexander the Sophist; cf. Jacques Jouanna, 'La postérité de l'embryologie d'Hippocrate dans deux traités pseudo-hippocratiques de la médecine tardive: "Sur la formation de l'homme et Sur la génération de l'homme et la semence", in Luc Brisson et al. (eds), L'embryon: formation et animation (Paris: Vrin, 2008), 15-41: 41.

16 The introduction coincides with fragment 93 attributed to Libanius; cf. Richard Foerster, Libanii opera (Lepzig: Teubner, 1922), Vol. 11, 3-5; cf. Diels II.57.

17 The text contains details on plebotomy and various simple drugs, recipes of composite drugs, and therapeutic recommendations on bites of venomous animals and poisons.

18 The text mainly contains remedies consisting of spells.

19 Cf. Diels II.37, III.50; and Stefan Weinstock, Catalogus codicum astrologorum Graecorum. V. Codicum Romanorum, pt. 4 (Brussels: Regia Academia Belgica, 1940), 156-63.

${ }^{20}$ The text contains details on various simple drugs and recipes of composite drugs in vernacular.

21 The following chapters, according to the numbering by Barbara Zipser, John the Physician's Therapeutics: A Medical Handbook in Vernacular Greek (Leiden: Brill, 2009), are missing: 16-21, 55-66, 68-73, 134-7, 143-6, 242-53.

22 It contains texts on diagnosis of pulse and urines, and details on phlebotomy; there are also recipes of composite drugs and remedies with superstitious connotations including spells. Various excerpts have been edited by Barbara Zipser, 'Magic, infidelity, and secret annotations in a Cypriot manuscripts of the early fourteenth century (Wellcome MSL.14)', in Steven M. Oberhelman (ed.), Dreams, Healing, and Medicine in Greece from Antiquity to the Present (Farnham: Ashgate, 2013), 251-68: 257-8, 261.
} 
A.

ca. 1350 (from watermarks).

Linn. 18 [ca. $113 \times 72$ ], unruled.

Original order of leaves: pp. 1-81, 84-119, 122, 123, 120, 121, 126, 127, 124, 125, 128-271, 82, 83.

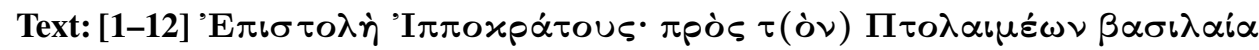

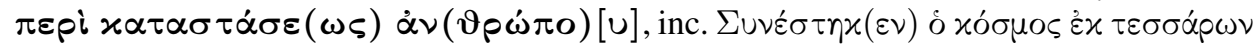

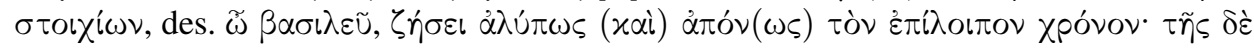

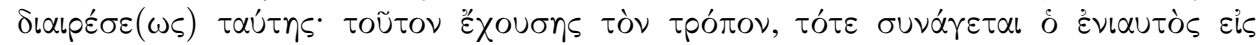

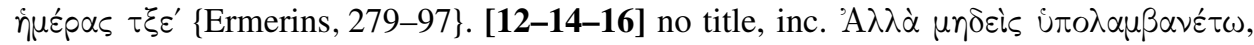

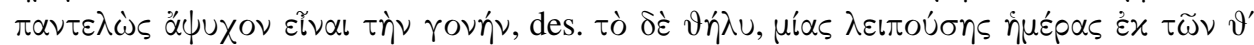

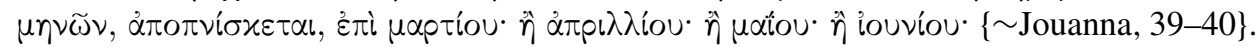

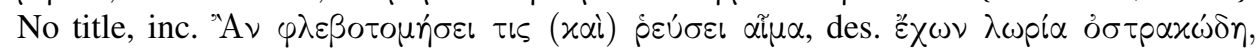

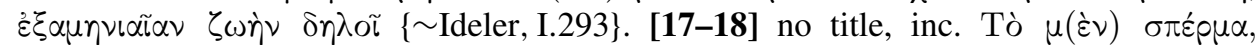

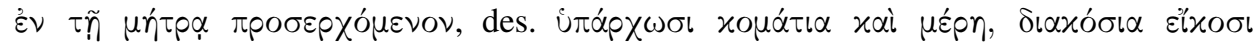

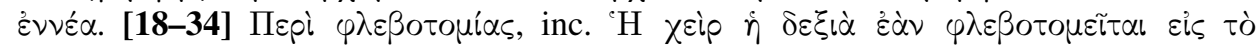

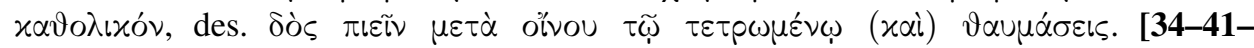

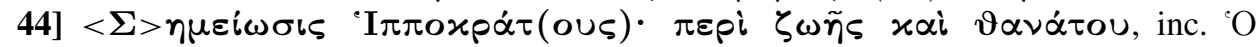

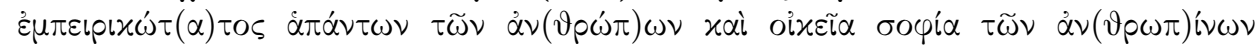

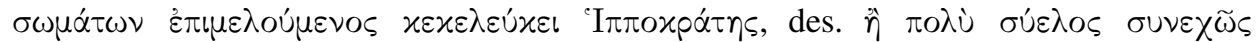

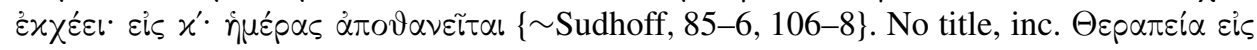

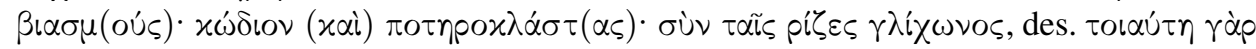

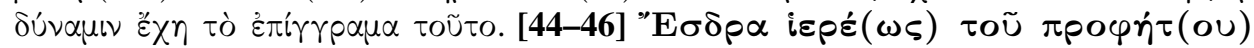

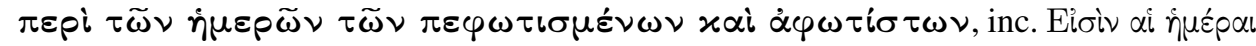

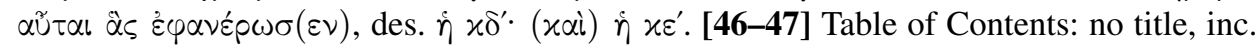

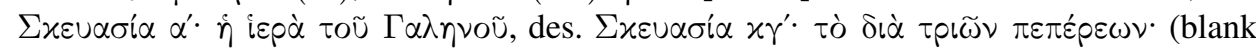

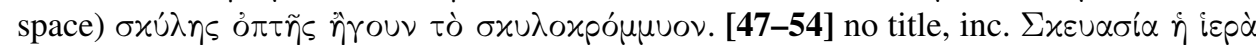

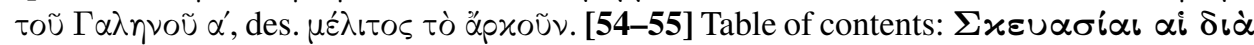

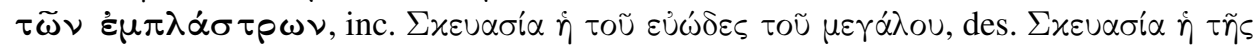

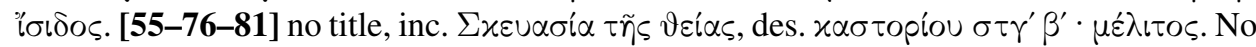

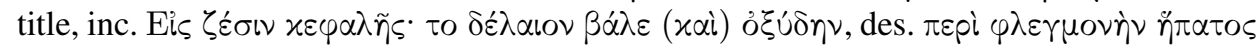

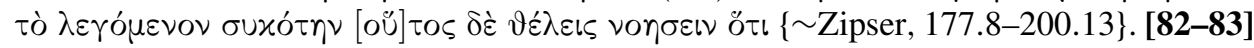

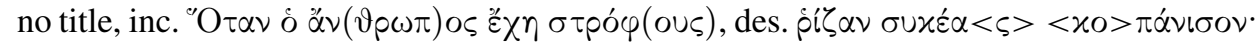

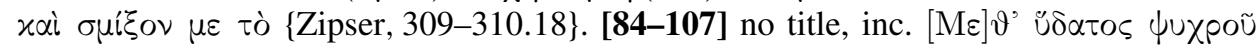

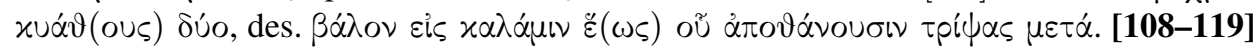

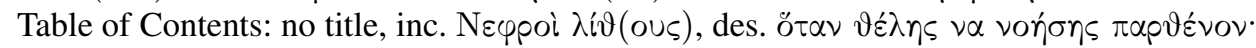

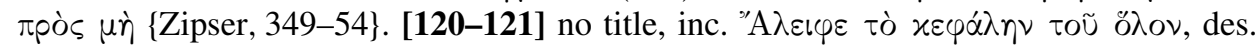

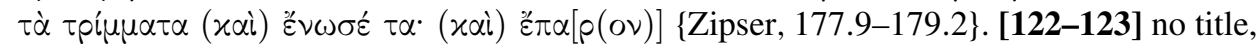

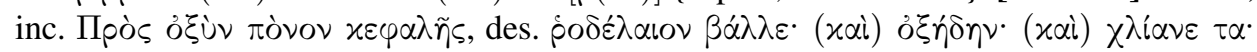

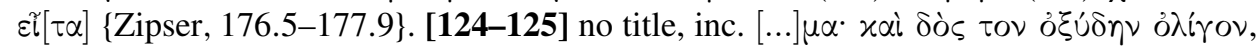

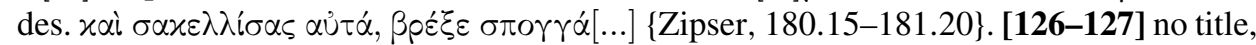

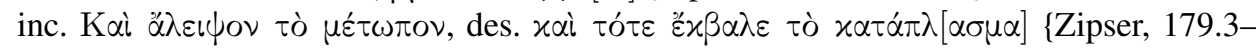

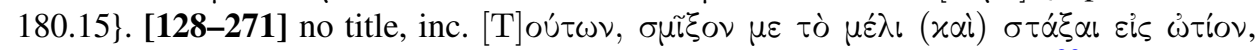

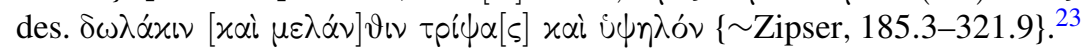

${ }^{23}$ Franz Zacharias Ermerins, Anecdota medica Graeca e codicibus MSS. expromsit (Leiden: S. et J. Luchtmans, 1840); Jouanna, op. cit. (note 15); Julius Ludwig Ideler, Physici et medici Graeci minores, 2 vols (Berlin: 
Handwriting: identified by Agamemnon Tselikas as a fourteenth-century Cypriot hand.

Annotations (non-scribal): (36, 37, 38, 39) Latin marginal notes in Gothic script,

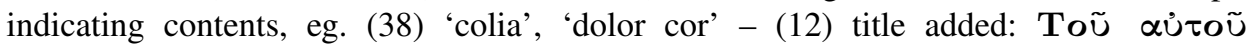

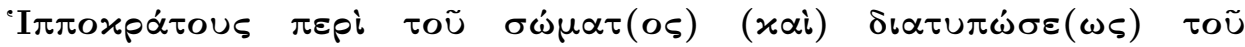
$\dot{\alpha} \nu(\vartheta \rho \dot{\omega} \pi) o u$.

Paper: folded in $8^{\circ}$; chain lines unclear; watermarks very similar to Mošin and Traljić 1900 (cercle), attested in $1352 .^{24}$

B.

ca. 1320-1340 (based on the watermarks and the attribution of the handwriting).

Linn. 11 [ca. $102 \times 70]$, unruled.

Original order of leaves: pp. 272-279, gap, 282-283, 280-281, gap, 284-289, 292-293, 290-291, 294-297, gap, 298-305, gap, 306-317.

Note: pp. 284-317 have non-scribal Greek numbering in the lower margin; many leaves seem to be missing, so that, for example, $\lambda \alpha$ (296) is followed by $\mu \beta$ (298).

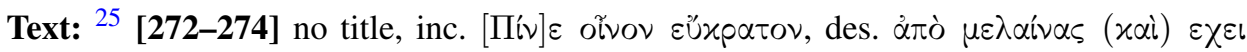

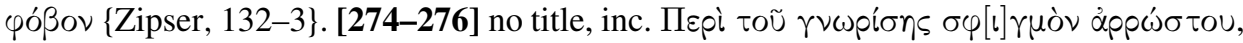

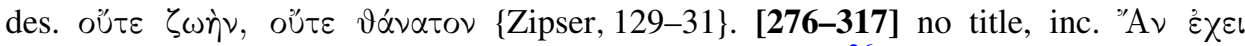

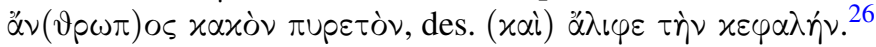

Handwriting: very similar (according to Barbara Zipser) to that of Theodoulos Philagres, active in Cyprus in the early fourteenth century. ${ }^{27}$

Text added by later hands: (274-5) apotropaic formulae in lower margins, eg. ' $\sigma 0 \pi \iota /$ $\pi \iota / \pi \iota^{2} 28$ - (275-86) remedies, including mostly Christians charms in invisible ink, ${ }^{29}$ eg.

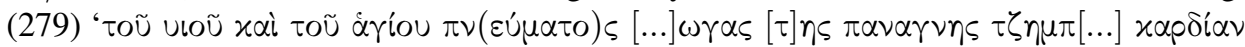

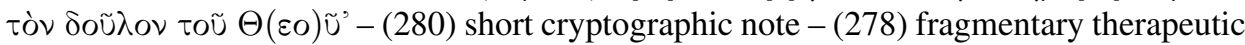

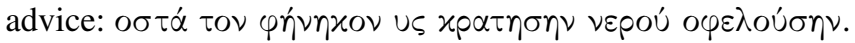

Paper: folded in $8^{\circ}$; chain lines unclear; watermarks (1) possibly similar to Mošin and Traljić 4642-4727 (hache) and (2) very similar to Mošin and Traljić 2803 (cloche), attested in $1336-9 .^{30}$

G. Reimer, 1841-42); Karl Sudhoff, 'Die pseudohippokratische Krankheitsprognostik nach dem Auftreten von Hautausschlägen, "Secreta Hippocratis" oder "Capsula eburnea” benannt', Archiv für Geschichte der Medizin, 9 (1915), 79-116; Zipser, op. cit. (note 21).

24 Vladimir Mošin and Seid Traljić, Filigranes des XIIIe et XIVe siècles, 2 vols (Zagreb: Académie yougoslave des sciences et des beaux-arts, 1957).

25 The text is lacunose and not clearly segmented. I have subdivided it in correspondence with Zipser's partial edition.

26 Barbara Zipser, 'Die uneinheitliche Überlieferung eines Fragmentes über den Puls und ein Iatrosophion', Galenos, 2 (2008), 129-34.

27 Barbara Zipser, op. cit. (note 21), 16. Cf. Costas Constantinides and Robert Browning, Dated Manuscripts from Cyprus to the Year 1570 (Washington DC: Dumbarton Oaks, 1993), pl. 44 (no. 30).

28 Edited by Zipser, see Nutton and Zipser, 264 (no. 21).

29 Some of them partly edited by Zipser, op. cit. (note 22), 264.

${ }^{30}$ Mošin and Traljić, op. cit. (note 24). 
$A$ and $B$.

Quires: $6 \times 8$ (p. 95), 4 (p. 105), 1 (p. 107), 6 (p. 119), 4 (p. 127), 6 (p. 139), $2 \times 8$ (p. 155), 6 (p. 167), 8 (p. 183), 7 (p. 213, no text lost), 8, 6, 8, 6; 6, 7, 4, 6.

Binding: corio russico, same tooling as the covers of London, British Library, Add. MS 5119. - Flyleaves: chain distance $22 \mathrm{~mm}$; watermark (only upper half preserved) similar to Gravell and Miller 476 (horn), attested in $1779 .{ }^{31}$

Marks of ownership: (front pastedown) label inscribed 'D.a.11'- (front flyleaf IVr) 'vid(e) Fabricii Bibl(iotheca) Graec(a) Vol. 12. p. 781 [ubi] mentionem facit primae Epistolae huius codicis' - (front flyleaf IVr) 'a a / a / I'.

Provenance: Anthony Askew (1722-74), London; [his sale, G. Leigh and J. Sotheby, London, 15 March 1785, lot 578]; purchased by James Sims (1741-1820), London, in 1785; purchased by the London Medical Society in 1802; purchased by the Wellcome Library in 1984.

Bibliography: Bibl. Askev., 36 (no. 578); Cat. Med. Soc. 1803, 94; Med. Soc. 1829, 158; Nias, lv; Diels, I.40-41, 47; Dawson, 24; Weinstock, op. cit. (note 48), 33 (no. 55); Nutton, 26; Jouanna, op. cit. (note 15), 41; Zipser, op. cit. (note 26), 129-34; Touwaide, 538-9; Zipser, op. cit. (note 21), 15-7; Nutton and Zipser, 259-70; Zipser, op. cit. (note 22), 251-68.

\section{MS.MSL.52 A and B (olim HH i 21 and 22 / We 28 and 29)}

Paper, $215 \times 145, \mathrm{v}+202+\mathrm{i}$ and $\mathrm{i}+210+\mathrm{i}$ (foliated 1-202 and 203-282, 283, 284-292, 293-296, 297-306, 307-312, 312-321, 322-406).

[1r-43v] John Zacharias Aktouarios, On the Activities and Illnesses of the Psychic Pneuma and the Corresponding Mode of Living (TLG 3188.001). [44r-143v] Idem, On Urines (TLG 3188.002). [145r-332v] Idem, Medical Epitome, ${ }^{32}$ Books 1, 2 (TLG 3188.003), 3, first part of 6, 4, 5. [333r-v] Theophilos (Protospatharios) or Stephen, On the Differences among the Fevers (TLG 0746.001), excerpt on sweats. [333v-341r] Anonymus, On Pulse, in the form of questions and answers. ${ }^{33}$ [341r-345v] Paul of Aegina, Medical Epitome (TLG 0715.001), excerpt on pulse. [345v-346r] Short text on pulse. [346r-v] Aetios of Amida, Tetrabiblon (TLG 0718.005), excerpt on pulse. [346v-354r] Galen, On the Pulse for Beginners (TLG 0057.058), excerpt. [354v-361v] Theophilos (Protospatharios), On Excrements (TLG 0729.003). [361v-363r] Ps.-Hippocrates, Sayings about Life and Death. [363v] Anonymous collection of recipes for composite drugs. [364r-366v] Ps.-Galen, On Procurable Remedies (TLG 0530.029), excerpt. [366v-403v] Anonymus, On Acute and Chronic Diseases (TLG 0721.019).

31 Thomas L. Gravell and George Miller, A Catalogue of Foreign Watermarks Found on Paper Used in America, 1700-1835 (New York: Garland, 1983).

32 Books 3-6 have no Greek edition yet and have only been published in Latin translation. The MS belongs to a family where the first part of Book 6 forms the second part of Book 3 and the second part of Book 6 comes after Book 5. The second part of Book 6 is missing in this MS.

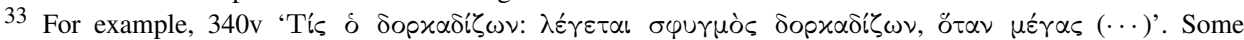
parts of the text on the first folia represent a synopsis of Galen's On the Pulse for Beginners, Karl Gottlob Kühn, Claudii Galeni Opera omnia, 20 vols (Leipzig: Carl Cnobloch, 1821-33), Vol. 8, 453-92. From folio $338 \mathrm{r}$, the text is similar to the anonymous synopsis on pulses published by Charles Victor Daremberg and Charles-Émile Ruelle, Oeuvres de Rufus d'Éphèse (Paris: J.-B. Baillière, 1879), 219-32: (338r.15-341r.112) inc.

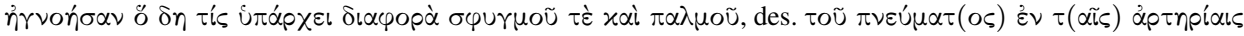

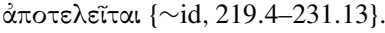


Note: This MS consists of two volumes bound separately but foliated continuously. Volume 52B comprises two distinct parts, 52B1 and 52B2. 52A and 52B1 are the work of the same scribe and were originally bound together. These are described below under $\mathbf{I}$, while 52B2 (ff. 333-403) is described under II.

I.

Constantinople, shortly before 1463 AD (from the note on f. 332v).

(Ff. 1-43) linn. 25 [ca. $173 \times 107$ ], unclear ruling; (ff. 44-332) linn. 25 [167 ×97], unclear ruling.

Text: [1r-v] Table of Contents: Toũ $\sigma o \varphi \omega \tau(\dot{\alpha}) \tau(o u)(\varkappa \alpha i) \lambda o \gamma \iota \omega \tau(\dot{\alpha}) \tau(o u)$

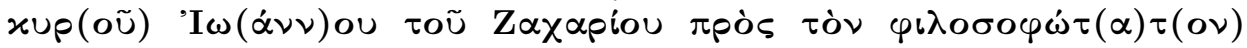

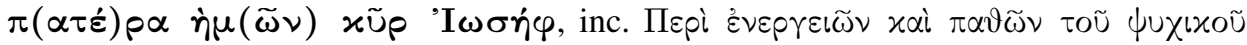

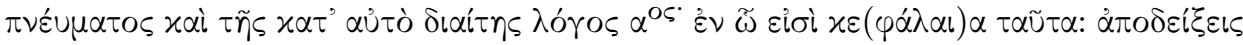

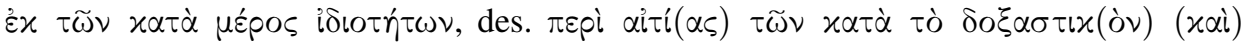

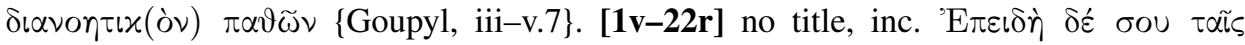

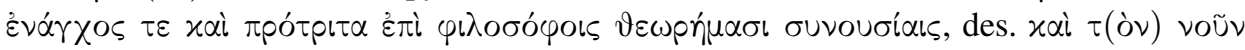

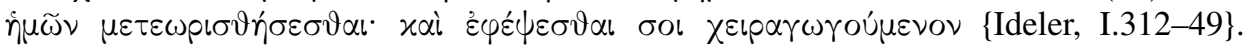

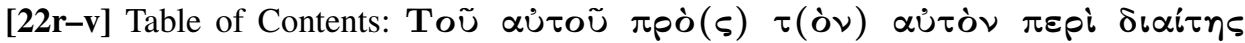

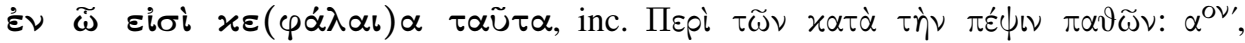

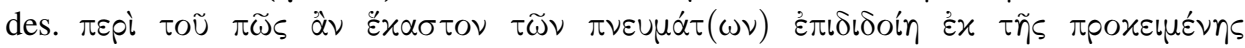

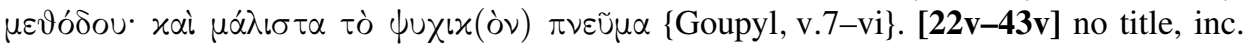

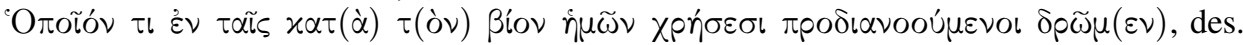

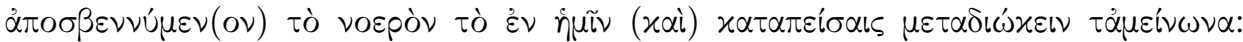

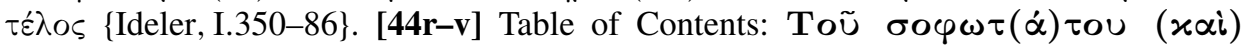

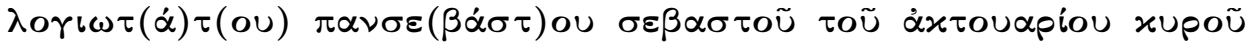

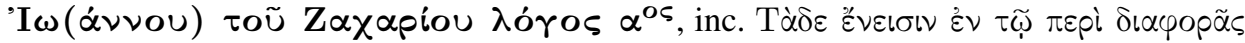

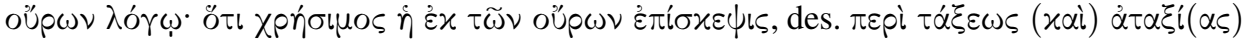

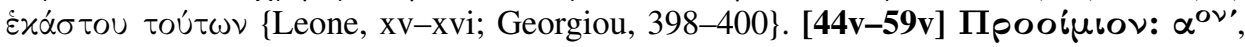

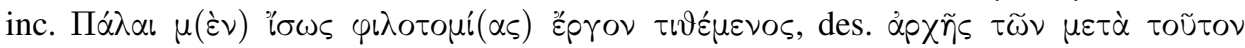

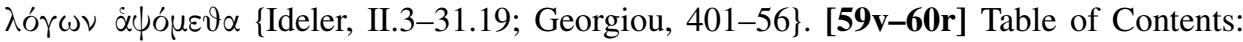

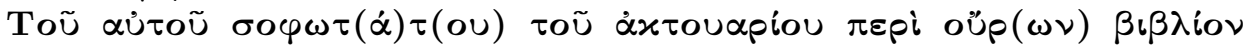

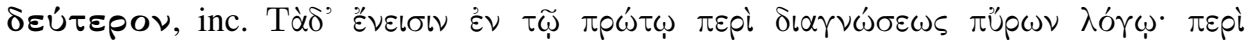

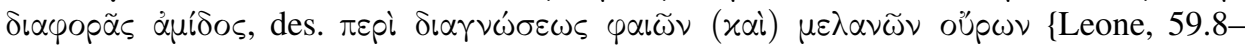

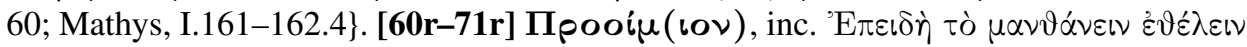

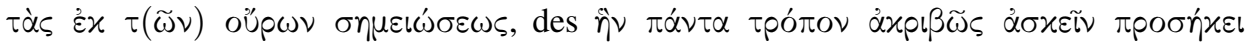

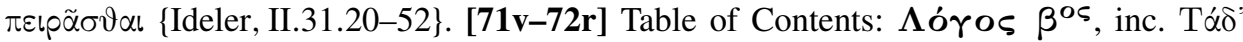

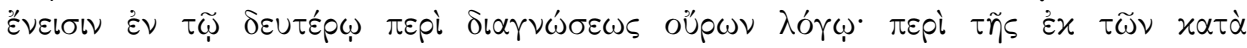

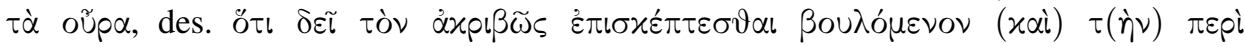

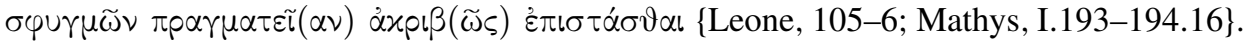

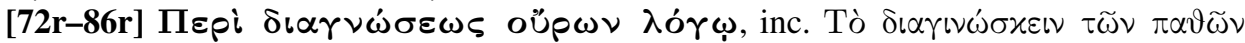

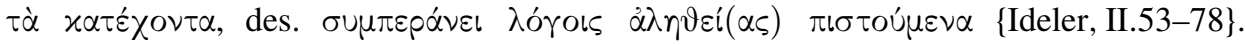

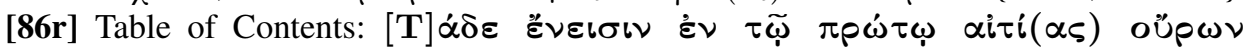

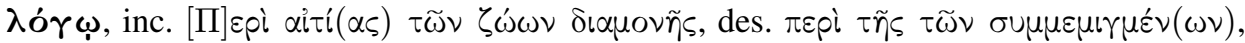

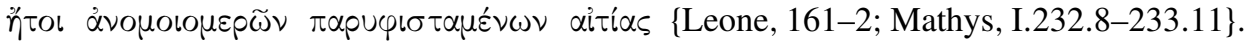

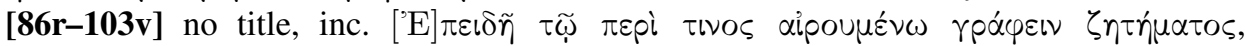

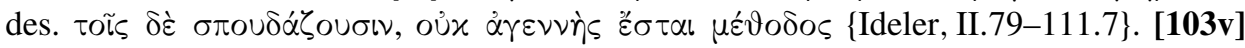

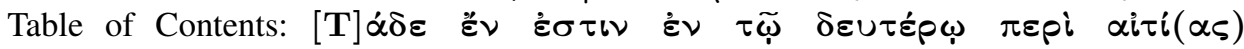




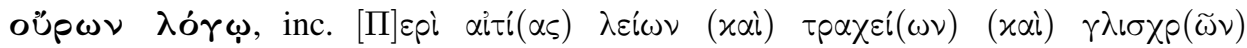

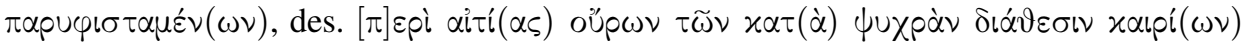

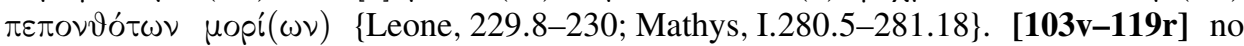

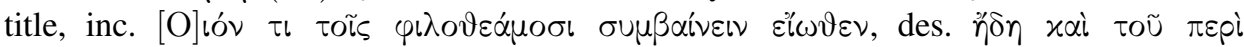

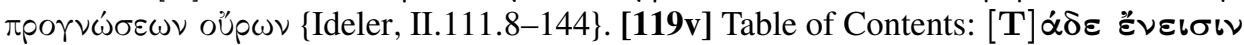

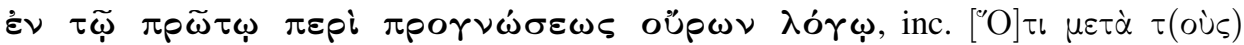

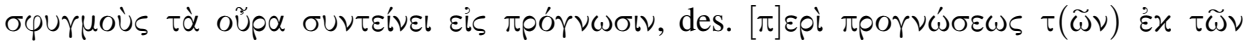

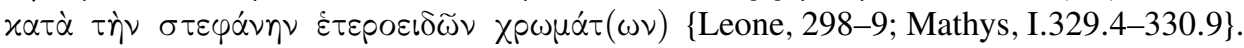

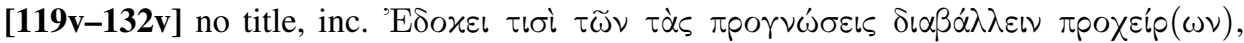

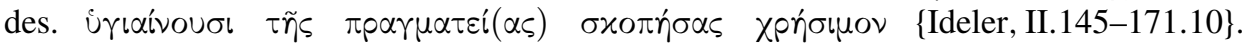

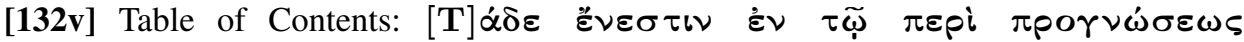

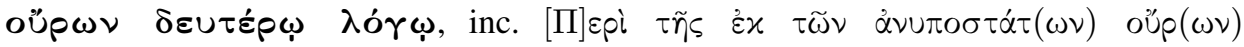

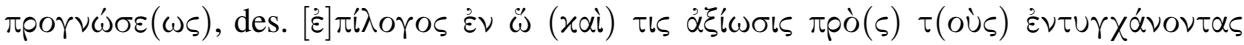

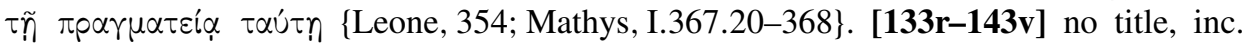

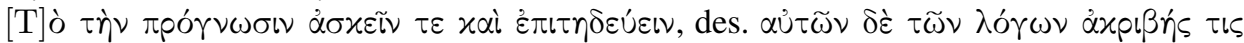

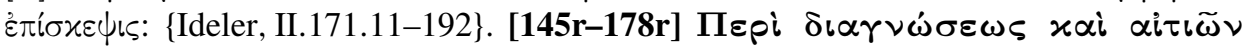

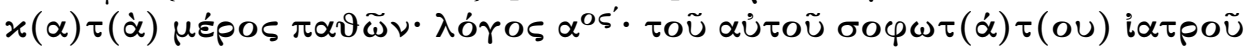

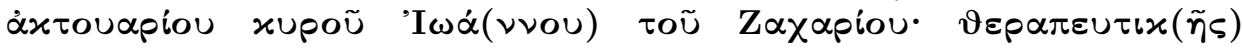

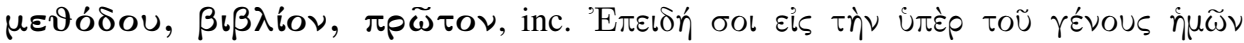

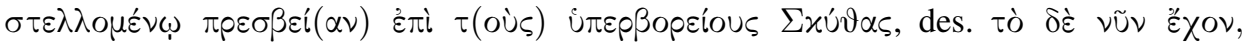

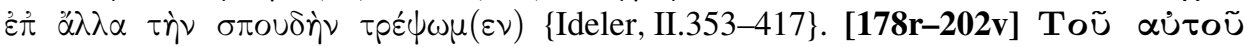

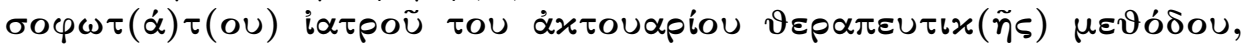

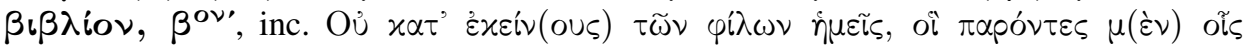

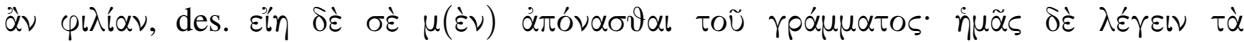

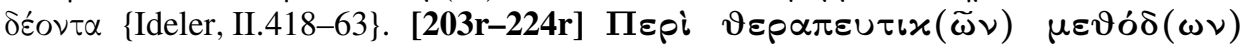

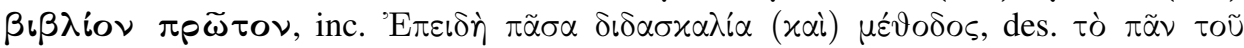

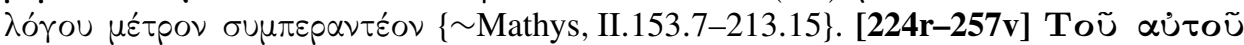

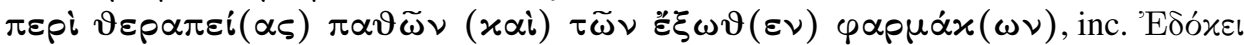

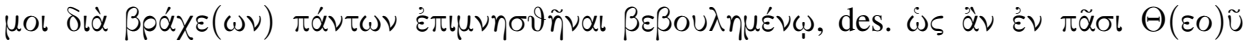

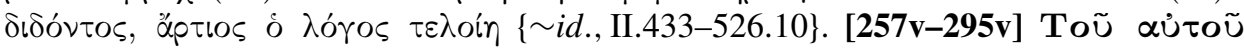

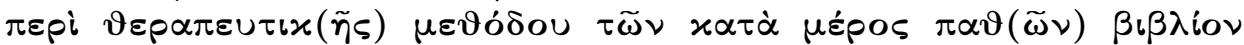

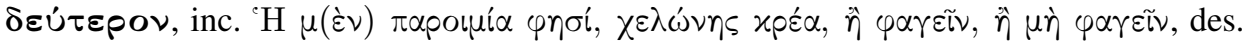

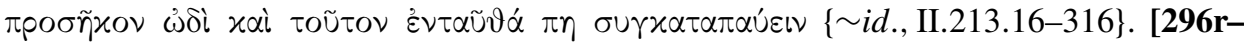

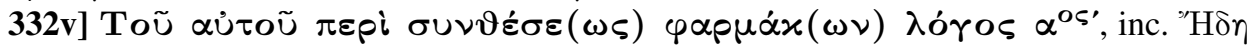

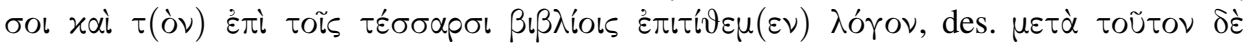

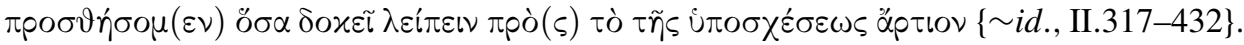

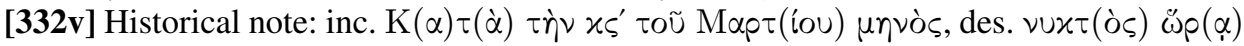

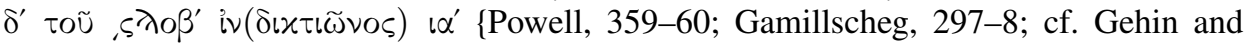
Kouroupou, I.410-11 (no. 172)\}. ${ }^{34}$

\footnotetext{
34 Jacques Goupyl, Actuarii De actionibus \& affectibus spiritus animalis, eisque victu, libri II (Paris: Martinus Juvenus, 1557); Ideler, op. cit. (note 23); Ambrogio Leone (trans.), De Urinis Actuarii Ioannis, Zachariae filii, medici praestantissimi, libri VII (Basel: Andreas Cratandrus, 1529); Stavroula Georgiou, 'Edition critique, traduction et commentaire critique du livre 1 "De Urinis" de Jean Zacharias Actouarios' (unpublished PhD thesis: Paris, École Pratique des Hautes Études, 2013); Corneille Henri Mathys (trans.), Actuarii Ioannis filii Zachariae Opera: De actionibus et spiritus animalis affectibus eiusque nutritione lib. II, De urinis lib. VII, Methodi medendi lib. V (Paris: Bernardus Turrisanus, 1556); J. Enoch Powell, 'Die letzten Tage der Grosskomnenen', Byzantinische Zeitschrift, 37 (1937), 359-60; Ernst Gamillscheg, 'Der Kopist des Par. gr. 428 und das Ende der Grosskomnenen', Jahrbuch der Österreichischen Byzantinistik, 36 (1986), 287-300; repr. in
} 
Annotations (possibly scribal): (32v, 34r, 46v, 47r, 49v, 50v, 51v, 54r, 56v, 59r, 63r, 65v, 66v, 70r, 74v, 76v, 78v, 80v, 85r, 85v, 88v, 93r, 93v, 95v, 96r, 96v, 98r, 99v, 100r, $100 \mathrm{v}, 103 \mathrm{r}, 105 \mathrm{r}, 108 \mathrm{r}, 109 \mathrm{v}, 110 \mathrm{v}, 112 \mathrm{v}, 114 \mathrm{r}, 115 \mathrm{v}, 116 \mathrm{r}, 116 \mathrm{v}, 117 \mathrm{v}, 118 \mathrm{r}, 118 \mathrm{v}, 119 \mathrm{r}$, $119 \mathrm{v}, 120 \mathrm{r}, 121 \mathrm{r}, 121 \mathrm{v}, 124 \mathrm{v}, 125 \mathrm{r}, 125 \mathrm{v}, 126 \mathrm{r}, 126 \mathrm{v}, 127 \mathrm{r}, 129 \mathrm{r}, 130 \mathrm{r}, 125 \mathrm{r}, 135 \mathrm{r}, 136 \mathrm{r}$, $136 \mathrm{v}, 137 \mathrm{r}, 140 \mathrm{r}, 140 \mathrm{v}, 141 \mathrm{v}, 143 \mathrm{r}, 143 \mathrm{v} 145 \mathrm{r}, 146 \mathrm{v}, 147 \mathrm{r}, 150 \mathrm{v}, 151 \mathrm{r}, 152 \mathrm{v}, 154 \mathrm{v}, 155 \mathrm{r}$, $155 \mathrm{v}, 157 \mathrm{v}, 158 \mathrm{r}, 158 \mathrm{v}, 159 \mathrm{v}, 160 \mathrm{v}, 161 \mathrm{r}, 162 \mathrm{v}, 164 \mathrm{r}, 165 \mathrm{r}, 169 \mathrm{r}, 169 \mathrm{v}, 174 \mathrm{v}, 176 \mathrm{v}$, $177 \mathrm{r}, 177 \mathrm{v}, 181 \mathrm{v}, 182 \mathrm{v}, 186 \mathrm{r}, 187 \mathrm{r}, 192 \mathrm{v}, 193 \mathrm{v}, 195 \mathrm{v}, 196 \mathrm{r}, 198 \mathrm{r}, 199 \mathrm{v}, 201 \mathrm{r}, 202 \mathrm{v}, 203 \mathrm{r}$, 204r, 205r, 206r, 206v, 209v, 207v, 220v, 256v, 269v, 273v, 282bis v, 307bis r, 313v, $321 \mathrm{bis} r$ ) correction or additions to the main text, variant readings, short explanations

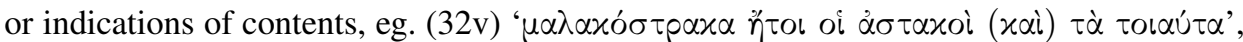

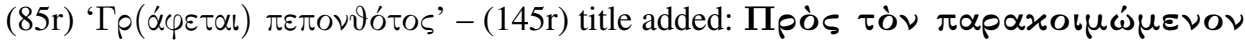

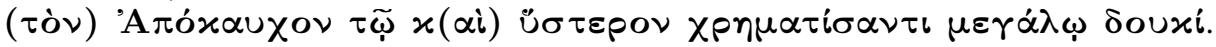

Illustrations (possibly scribal): (54r) diagram of the urine vial divided into eleven areas,

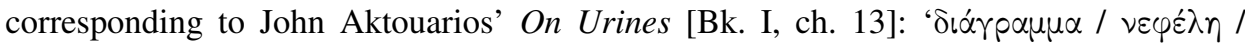

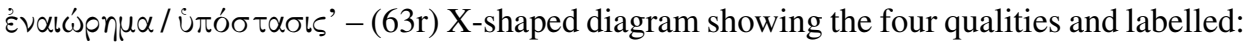

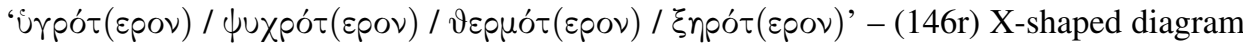

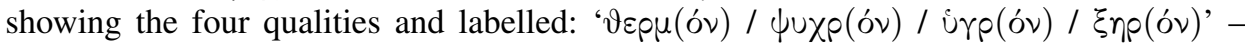

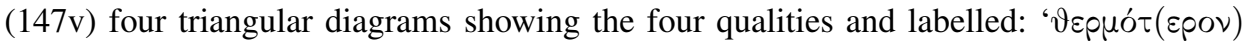

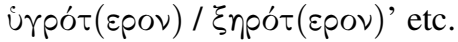

Handwriting: attributed by Brigitte Mondrain to Demetrios Angelos.

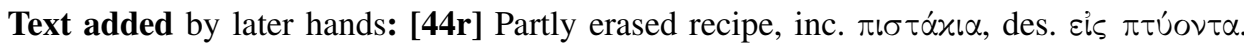

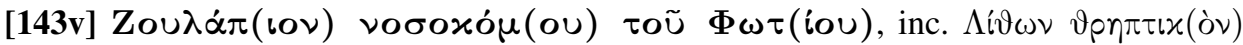

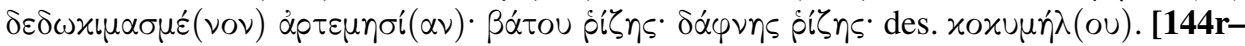

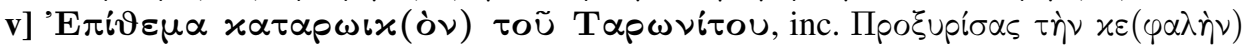

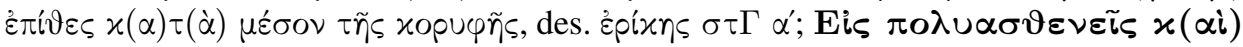

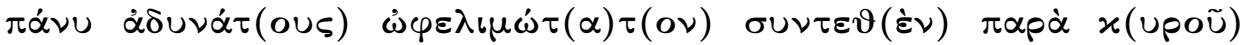

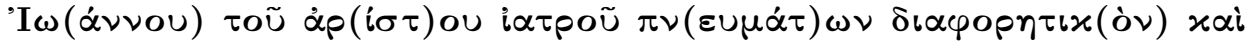

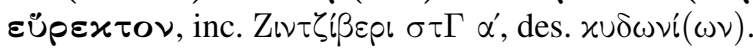

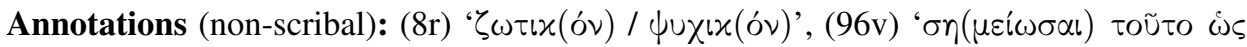

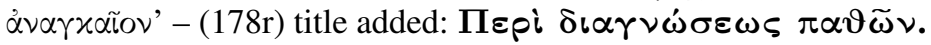

Paper: folder in $4^{\circ}$; chain distance $33 \mathrm{~mm}$; watermark similar to Piccard 122469 (scissors), attested in 1455 .

Quire signatures: (ff. 1-43) non-scribal, Greek numerals Iel $\beta$ (9r) through $\zeta(41 \mathrm{r})-$ (ff. 44-211) scribal, Greek numerals Iel (except for $\alpha^{\prime}$ Ii3 on f. 51v) $\alpha^{\text {ov }}$ (51v) through $x \alpha^{(o v)}(204 r)-(f f .212-332)$ possibly scribal, Greek numerals Iel $\alpha$ (21r) through เ $\sigma \tau$ (326r).

II.

Eastern Mediterranean, ca. 1445 (from watermarks)

Original order of leaves: 333-379, 388-395, 380-387, 396-403.

idem, Manuscripta Graeca: Studien zur Geschichte des griechischen Buches in Mittelalter und Renaissance (Purkersdorf, 2010) [Codices manuscripti, Supplementum 3], 190-202; Paul Géhin and Matoula Kouroupou, Catalogue des manuscrits conservés dans la Bibliothèque du Patriarcat Oecuménique: les manuscrits du monastère de la Panaghia de Chalki, 2 vols (Turnhout: Brepols, 2008). 


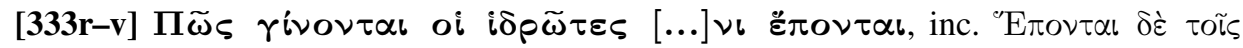

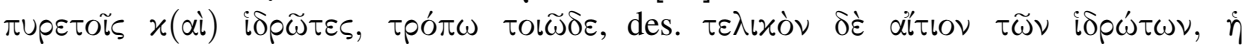

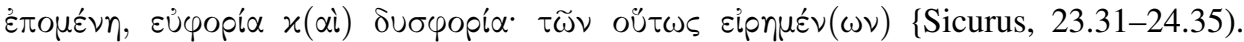

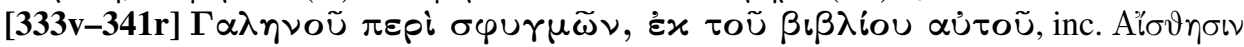

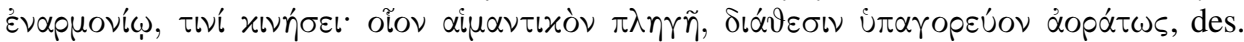

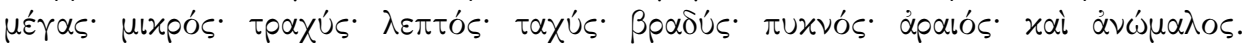

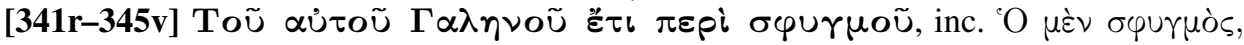

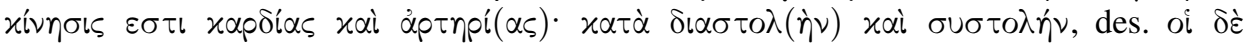

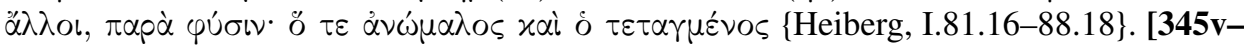

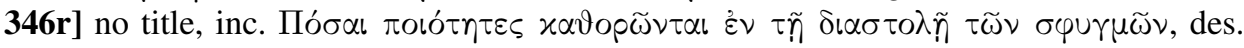

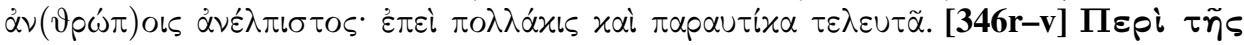

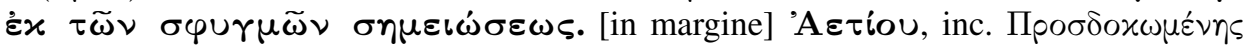

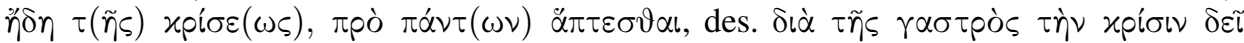

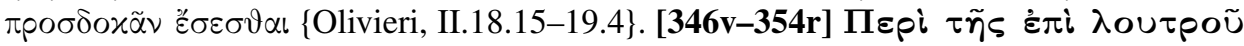

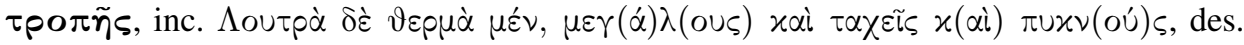

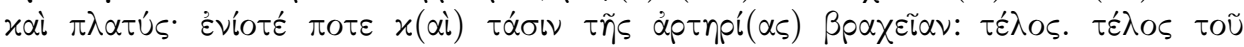

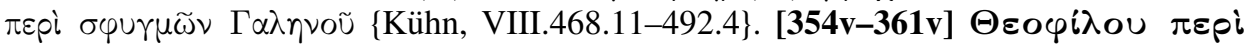

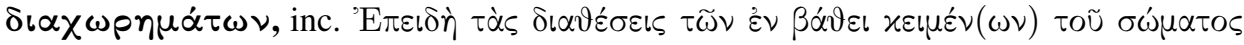

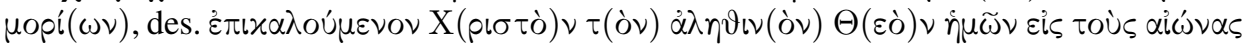

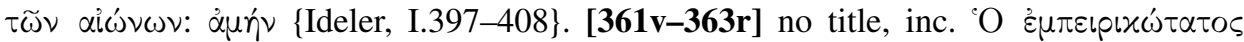

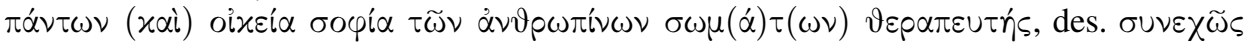

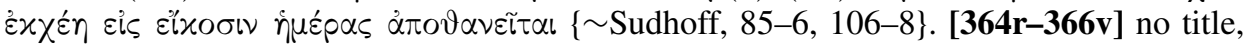

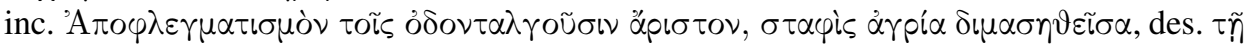

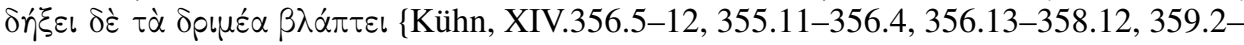

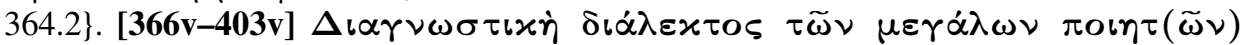

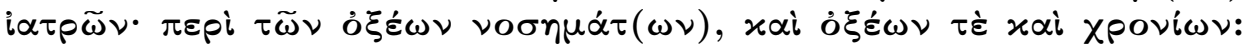

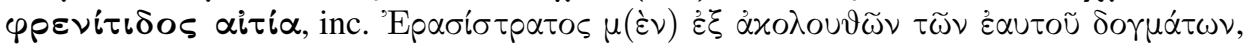

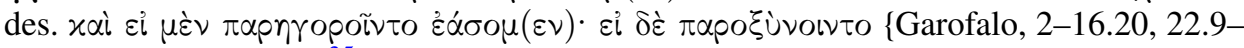
$136.6,140.13-174.23\} .^{35}$

Blank pages: $404 \mathrm{v}$.

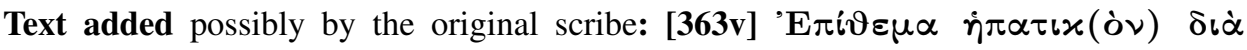

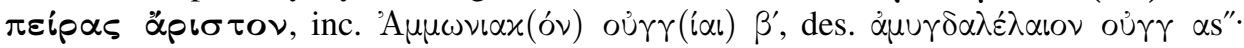

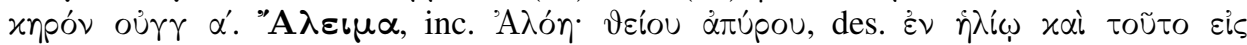

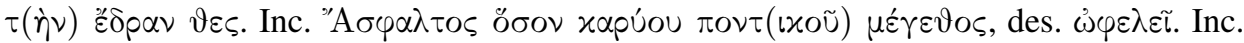

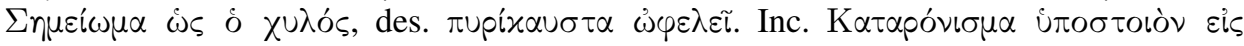

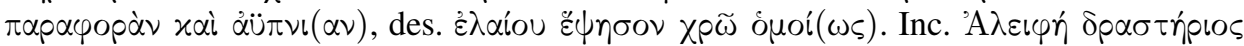

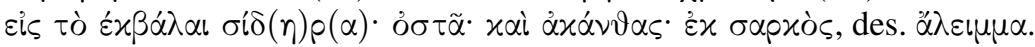

Annotations (scribal): (334r, 334v, 335r, 337r, 339v, 348r, 348v, 351v, 354v, 355r, 356r, 357v, 357r, 357v, 358r, 359r, 363v, 365v, 367r, 368v, 370r, 373v, 377v, 378r, 380v, 381r, $384 \mathrm{v}, 385 \mathrm{v}, 387 \mathrm{r}, 392 \mathrm{v}, 396 \mathrm{r}, 396 \mathrm{v}, 397 \mathrm{v}, 401 \mathrm{v}$ ) additions or corrections to the main text,

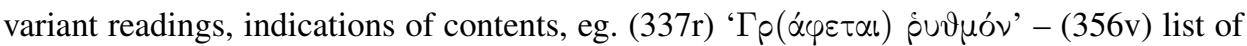

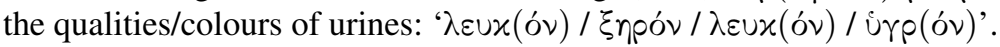

35 Demetrius Sicurus, Theophili et Stephani Atheniensis De febrium differentia ex Hippocrate et Galeno (Florence: Bencini, 1862); Johan Ludvig Heiberg, Paulus Aegineta, 2 vols (Leipzig: Teubner, 1921-24), [CMG, IX/1-2]; Alexander Olivieri, Aetii Amideni libri medicinales, 2 vols (Leipzig: Teubner, 1935-50), [CMG, VIII/ 1-2]; Kühn, op. cit. (note 33); Ideler, op. cit. (note 23); Sudhoff, op. cit. (note 23); Ivan Garofalo (ed.), Anonymi medici De morbis acutis et chroniis (Leiden: Brill, 1997). 
Handwriting: similar to those of (according to Georgi Parpulov) Constantine Triboles (RGK II 318) and (according to Rudolf Stefec) Mark (RGK III 437).

Recipes added by later hands: [333v] Inc. 'H

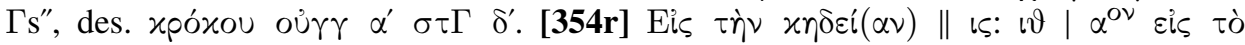

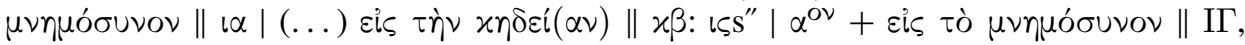

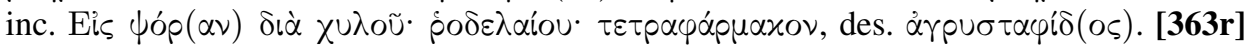

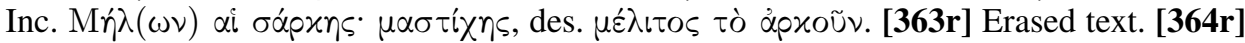

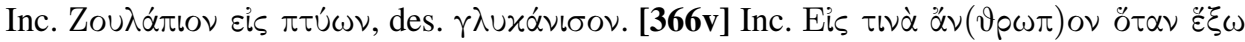

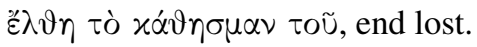

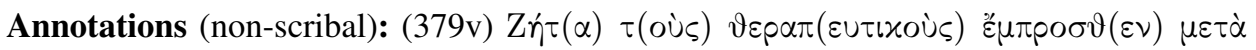

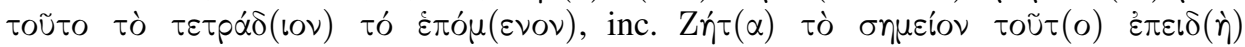

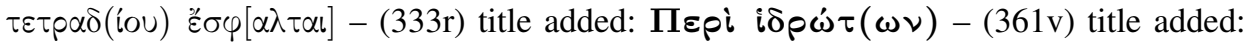

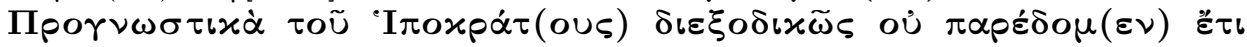
$\zeta \widetilde{\omega} \nu$ - (380r) Rhaz(eus) lib(er) XIX. cap(itulus) XX.

Illustration (non-scribal): (338v) partial x-shaped diagram of pulses.

Paper: folder in $4^{\circ}$; chain distance $38 \mathrm{~mm}$; watermark very similar to Piccard 122600 (anvil), attested in 1444 .

Quire signatures (non-scribal): Greek numerals $\operatorname{Im} 1$ (except for $\alpha^{\text {ov }} \operatorname{Im} 3$ on f. 399v) $\beta^{\text {ov }}$ (340r) through $\zeta(388 r)$.

\section{$A$ and $B$.}

Quires: $5 \times 1$ (v), $5 \times 8$ (f. 40), $3 \times 1$ (f. 43); $25 \times 8$ (f. 243 ), 10 (f. 253 ), $10 \times 8$ (f. 325), $1+6$ (f. 332; no text lost); $1+6$ (f. 333), 6+1 (f. 339), $8 \times 8$ (f. 403), 1 (f. 404), 2 (f. 406), 1 (i).

Binding: corio russico. - Pastdowns and outermost flylaves: machine made-paper. Flyleaves ii-iii in vol. 1 and 405-406: chain distance $25 \mathrm{~mm}$, unclear watermark. Flyleaves iv-v in vol. 1 and 404: chain distance $31 \mathrm{~mm}$, watermark very similar to Piccard $150772,150409,150636$ (three hills), attested in 1461-77.

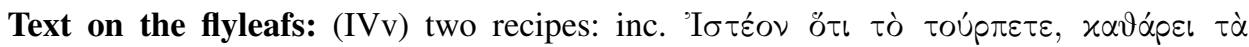

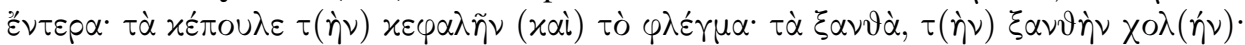

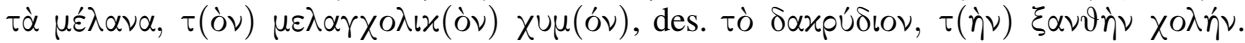

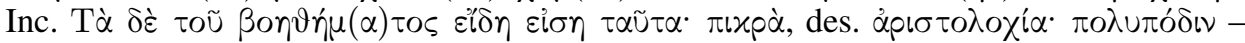

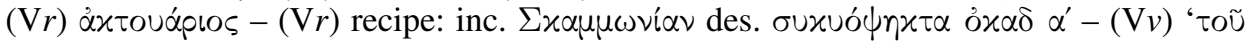

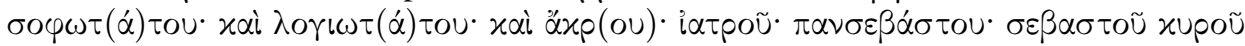

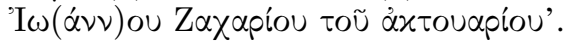

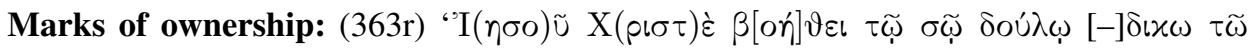
'Apoupo $\mu^{\tau \tau}[?]$ ' [the following three lines of text are completely blotted out] - (404r)

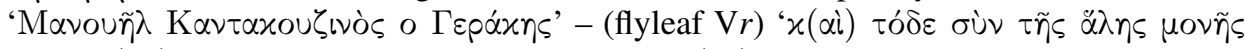

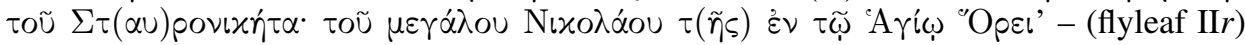
'Ex Bibliotheca Askeviana / P. ii. Art 540 / J. Sims'.

Provenance: Manuel Kantakouzenos Gerakes (cf. Wellcome MS.MSL.114) - Stavronikita Monastery, Mount Athos - brought to England in 1749 - Anthony Askew (1722-74), London; [his sale, G. Leigh and J. Sotheby, London, 15 March 1785, lot 540]; purchased by James Sims (1741-1820), London, in 1785; purchased by the London Medical Society in 1802; purchased by the Wellcome Library in 1984 . 
Bibliography: Bibl. Askev., 33 (no. 540); Cat. Med. Soc. 1803, 3; Cat. Med. Soc. 1829, 4; Daremberg, 158-9; Nias, liii-liv; Diels, I.41, 109, 131, II.7, 48, 102, 108-10, 131; Dawson, 59-60; Powell, op. cit. (note 34), 359-60; Gamillscheg, op. cit. (note 34), 287-300; Nutton, 24-6; Garofalo, op. cit. (note 35), xvii; Nutton and Zipser, 261; Touwaide, 538-9; Georgiou, op. cit. (note 34), 250-3, 364-7.

Georgios A. Costomiris, 'Études sur les écrits inédits des anciens médecins grecs. Cinquième série, XII ${ }^{\mathrm{e}}-\mathrm{XIV}^{\mathrm{e}}$ siècles: Jean Tzetzès, Nicolas Myrepsus, Jean Actuarius', Revue des Études Grecques, 10 (1897), 405-45: 441.

Donald Nicol, The Byzantine Family of Kantakouzenos (Cantacuzenus) ca. 1100-1460: A Genealogical and Prosopographical Study (Washington DC: Dumbarton Oaks, 1968) [Dumbarton Oaks Studies, 11], 128-129 with note 20.

Frank Stubbings, 'Anthony Askew's "Liber Amicorum", Transactions of the Cambridge Bibliographical Society, 6 (1976), 306-21: 317.

Brigitte Mondrain, 'Jean Argyropoulos professeur à Constantinople et ses auditeurs médecins, d'Andronic Eparque à Démétrios Angelos', in Georgios Makris and

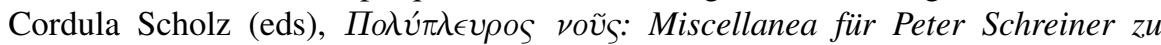
seinem 60. Geburtstag (Munich: Saur, 2000), 223-50: 236-7, 250.

eadem, 'Comment était lu Galien à Byzance dans la première moitié du XVe siècle? Contribution à quelques aspects de l'histoire des textes', in Antonio Garzya and Jacques Jouanna (eds), Trasmissione e ecdotica dei testi medici greci: Atti del IV Convegno Internazionale, Parigi 17-19 maggio 2001 (Naples: D’Auria, 2003), 361-84: 366.

eadem, 'Démétrios Angelos et la médecine: contribution nouvelle au dossier', in Véronique Boudon-Millot et al. (eds), Storia della tradizione e edizione dei medici greci: Atti del VI Colloquio internazionale, Paris 12-14 aprile 2008 (Naples: D’Auria, 2010), 293-322: 295, 299, 301, 305.

Petros Bouras-Vallianatos, 'Medical Theory and Practice in Late Byzantium: the Case of John Zacharias Aktouarios (ca. 1275-ca. 1330)' (unpublished PhD thesis: King's College London, 2015), 125, 346-8, 382.

\section{MS.MSL.60 (olim HH i 17 / We 30)}

Eastern Mediterranean, ca. 1450-1500 AD (from watermarks).

Paper, $213 \times 145$, iii + $221+$ iii (foliated 1-221), linn. 34 [160 × 90], unclear ruling.

[1r-11v] [Hippocrates], Aphorisms (TLG 0627.012). [12r-19r] [Hippocrates], Prognosticon (TLG 0627.003). [20r-45v] Epitome of Nicholas Myrepsos' Dynameron. ${ }^{36}$

\footnotetext{
36 The text has no Greek edition and has only been printed in a Latin translation by Leonhart Fuchs: Nicolai Myrepsi Medicamentorum opus (Basel: I. Opori, 1549). In contrast to the usual structure of the recipes given in the full version of the Dynameron such as in Parisinus gr. 2243 (AD 1339), where indication is followed by the list of substances and the method of preparation, here there is only the title followed by the list of ingredients for each recipe. This epitome comprises three parts: the first has, in no particular order, recipes from various parts of the Dynameron, the second focuses on oils, the third is an excerpt on plasters.
} 
[46r] Anonymous collection of questions and answers on the nature of man. ${ }^{37}$ [46rv] Anonymus, On the Creation of the World and Man (TLG 0721.006). [46v-48r] Anonymus, On Offspring (TLG 0627.024). ${ }^{38}$ [48r-50v] Anonymus, Lexicon of medical synonyms. ${ }^{39}$ [50v-53r] Paul of Aegina, Medical Epitome (TLG 0715.001), excerpt on the substitution of drugs. ${ }^{40}$ [53r-56r] Anonymous, collection of opuscules on various functions of the human body. [56r-57r] Ps.-Galen, On Weights and Measures (TLG 0530.022), excerpt. [57r-58r] Anonymus, On Stones. [58r] Ps.-Avicenna, Short prognostic remedy. [60r-62r] Anonymous excerpts on astrology followed by Easter tables. $^{41}$ [62v] Anonymous short excerpt on astrology. [62v] [Hippocrates], On the Seven Divisions of a Man's Life (TLG 0627.044), excerpt. [62v] Recipe for a composite drug attributed to Saint Gregory the Theologian. [62v] Anonymous short excerpt on the four elements. [63r] Anonymus, recipe on dysuria. [63r-71r] Anonymous collection of astrological and geographical excerpts. [71r] Anonymous opuscule on human conception. [71v] Anonymous bilingual [Greek and Arabic] lexicon of plant names. [73r-124v] Anonymous medical compilation on diagnosis and therapy of diseases following, generally, an a capite ad calcem structure. ${ }^{42}$ [125r-138r] Collection of recipes attributed to Demetrios Pepagomenos. [138r-v] Anonymous opuscule On Temperaments. [138v142v] Theophilos (Protospatharios) or Stephen, On differences between the fevers (TLG 0746.001), excerpt. ${ }^{43}$ [142v-162v] Symeon Seth, On the Capacities of Foodstuffs (TLG 3113.002). [163r-169v] Anonymous collection of opuscules on various medical matters. ${ }^{44}$ [170r-171r] Ps.-Galen, On the Distinction of Urines. [171r-177r] Theophilos (Protospatharios), On Urines (TLG 0729.002). [177r-184r] Anonymus, On Urines. [184r-185v] Anonymus, On Excrements. [185v-187v] Table of contents of John Zacharias Aktouarios' On Urines (TLG 3188.002). ${ }^{45}$ [187v-189r] Nikephoros Blemmydes, Canon on Urine Vials. ${ }^{46}$ [189r] Nikephoros Blemmydes, Canon on Phlebotomy.

37 The first half of the text bears similarities with the Ps.-Galenic Medical Definitions; cf. Kühn, op. cit. (note 33), Vol. 19, 355.7-11.

38 The text is same as the work printed by Ideler, op. cit. (note 23), Vol. 1, 294-6 up to 47v, 1.24: '(...) ( $\delta \dot{\varepsilon})$

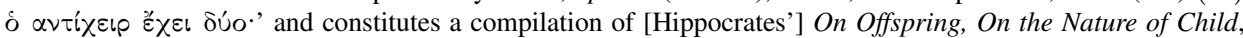
Émile Littré, Oeuvres complètes d'Hippocrate, 10 vols (Paris: J.-B. Baillière, 1839-61), Vol. 7, 470ff; here, the text carries on for 33 more lines. A slightly shorter version is also found in Parisinus gr. 2303, 83v-85r, and Parisinus gr. 2320, 1r-5r, and has been edited by Jouanna, op. cit. (note 15), 32-8.

${ }^{39}$ Ed. Luigi Tartaglia, 'Il lessico medico del codice Lond. Med. Soc. 60', in Antonio Garzya and Jacques Jouanna (eds), I testi medici greci - tradizione e ecdotica: Atti del III Convegno Internazionale, Napoli 15-18 ottobre 1997 (Naples: D'Auria, 1999), 547-57: 552-7; unfortunately, this edition skips the last five lines of text on fol. 50v.

40 The text here contains three more entries in addition to those in Heiberg, op. cit. (note 35), Vol. 2, 401-8.

41 Cf. Denis Petau, Uranologion sive Systema variorum authorum, qui de sphaera, ac sideribus, eorumque motibus Graecè commentati sunt (Paris: Sebastiani Cramoisy, 1630), $388 \mathrm{ff}$.

42 It is noteworthy that, among others, it contains recipes of clearly oriental origin attested in late Byzantine medical works such as Nicholas Myrepsos' Dynameron and John Zacharias Aktouarios' Medical Epitome, eg.

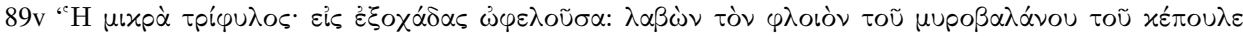
$(\ldots)$.

43 The chapters are usually abridged and their order varies largely in contrast to the version edited by Sicurus, op. cit. (note 35 ).

44 The opuscule on phlebotomy, 166r-169r, includes the term ' $\beta \alpha \sigma \iota \lambda \iota x$ ' $\varphi \lambda \varepsilon$ ' $\psi$ ', found only in fifteenth-century Byzantine medical manuscripts and probably denoting an influence from Islamic medicine; see Oswei Temkin, 'The Byzantine origin of the names for the basilic and cephalic veins', in idem, The Double Face of Janus and Other Essays in the History of Medicine (Baltimore: Johns Hopkins University Press, 1977), 198-201.

45 The short text included between the contents of Books 2 and 3 accompanies the diagram of a urine vial and corresponds with John's description of the urine vial in chapter 13 of Book 1.

46 The text is written in the form of ecclesiastical canon; some manuscripts attribute it to Maximos Planoudes; 
[189v-191r] Ps.-Hippocrates, On Urines Vials. [191r] Ps.-Galen, On the Urine Vial. [191r-v] On Urines attributed to the Persians (TLG 0721.013). [191v] Anonymous opuscule On the Sanguine Complexion (TLG 0721.003). [192r-v] Anonymous opuscule On the Four Elements of the Human Body. [192v] Anonymous opuscule On the Five Senses. [193r-198r] Ps.-Galen, On Pulse. [198r-v] Anonymous opuscule On the Qualities of Pulse. [198v-199r] Ps.-Galen, Medical Definitions (TLG 0530.041), excerpt on pulse. [199r-205v] Theophilos (Protospatharios), On Pulse (TLG 0729.004). ${ }^{47}$ [206r-220r] Ps.-Galen, On Procurable Remedies (TLG 0530.029), Book 1. [220r-222v] Anonymous collection of recipes for compound drugs.

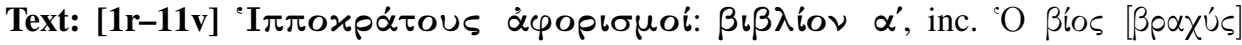

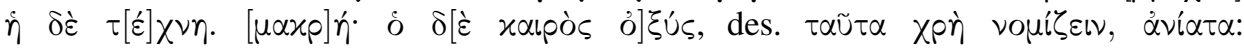

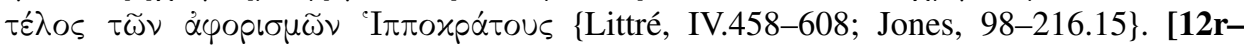

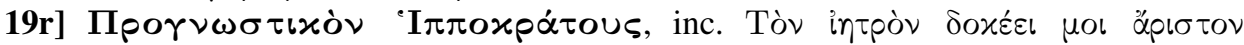

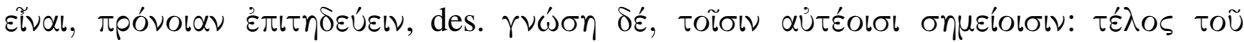

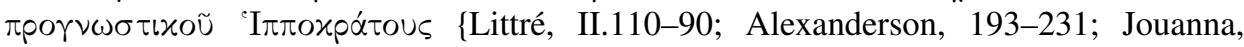

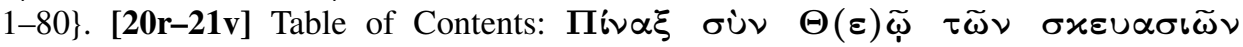

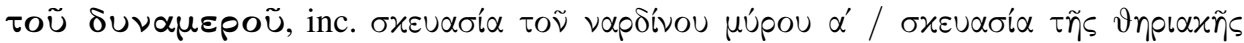

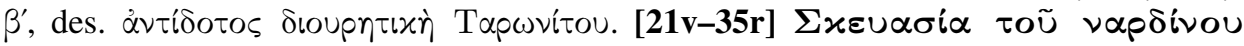

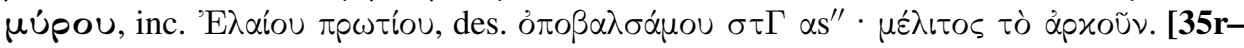

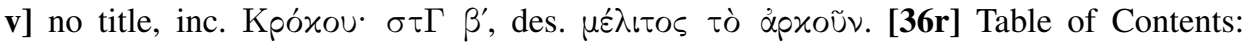

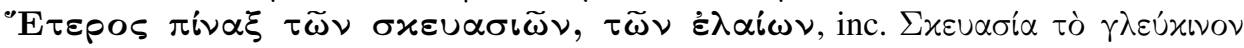

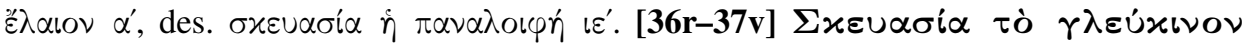

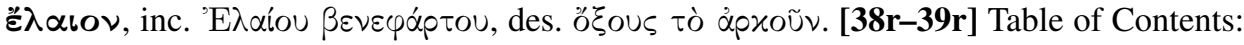

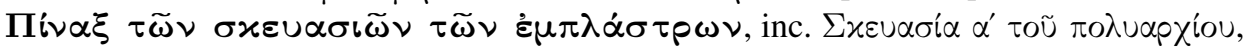

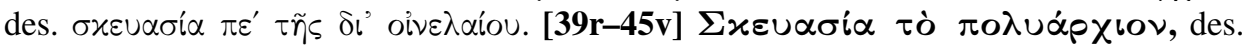

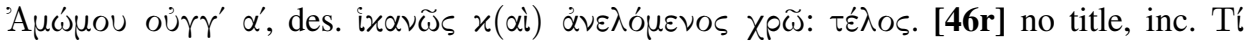

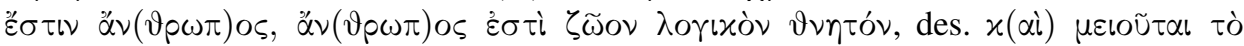

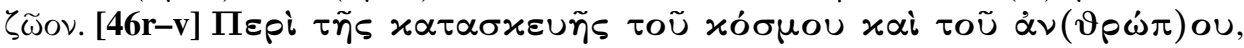

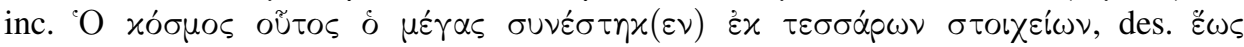

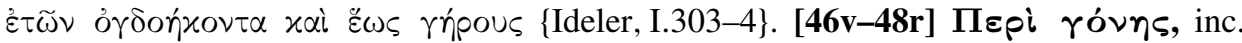

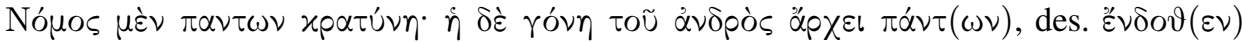

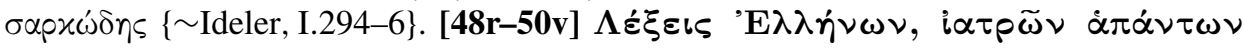

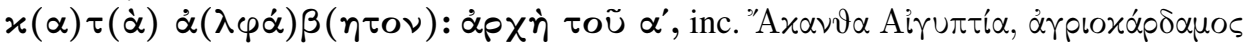

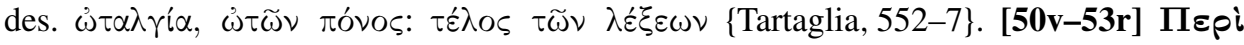

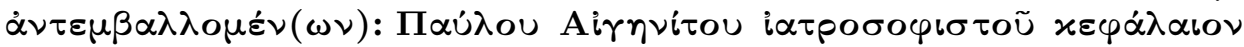

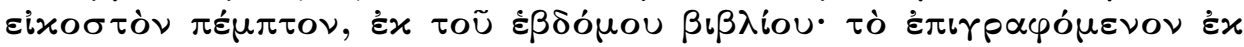

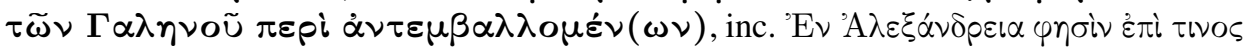

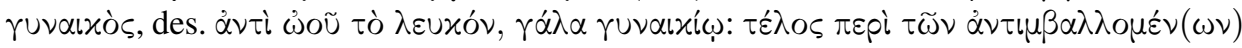

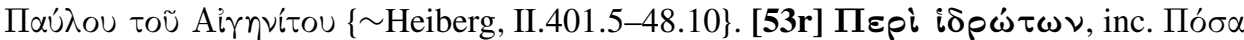

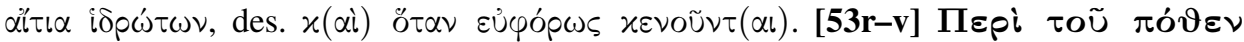

cf. Charle du Cange, Glossarium ad scriptores mediae et infimae Graecitatis (Paris: Hubertus Welter, 1688) Vol. 2, 1629, s.v. Ué

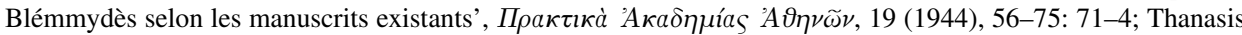

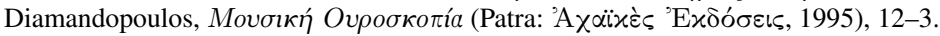

47 Some parts of the text are abridged; from 200v the text follows very closely Ermerins' edition, op. cit. (note 23), 33.14ff, with occasional gaps; the text here is sometimes supplemented with material not found in Ermerins' edition; there is no diagram on pulses. 


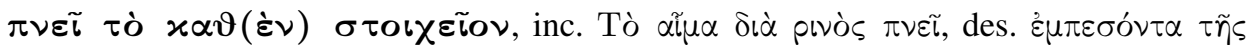

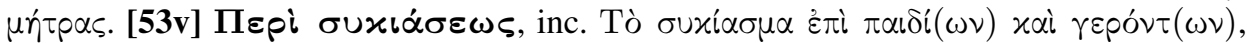

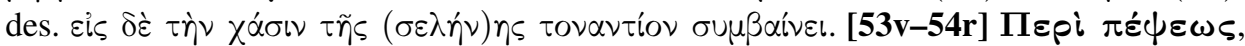

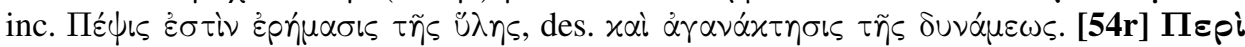

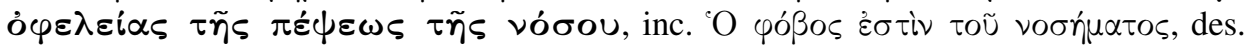

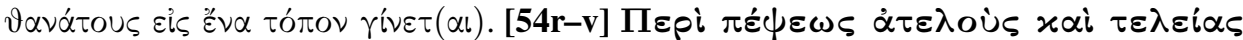

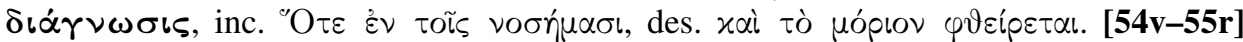

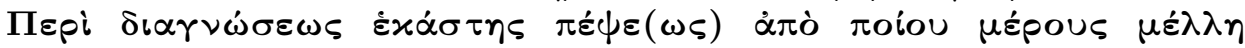

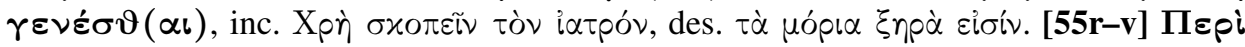

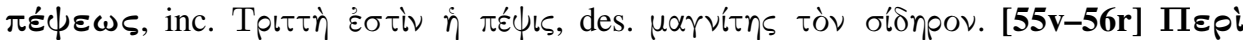

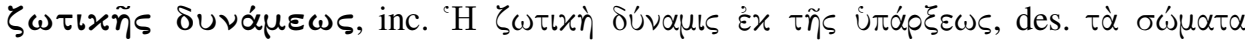

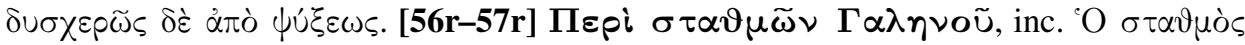

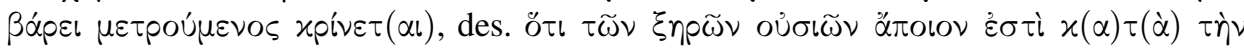

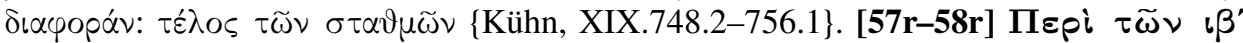

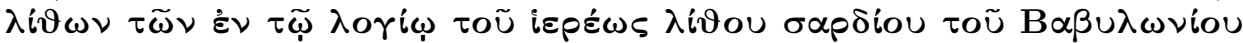

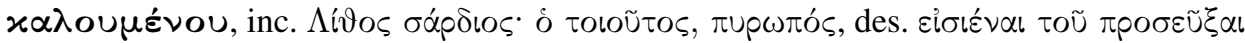

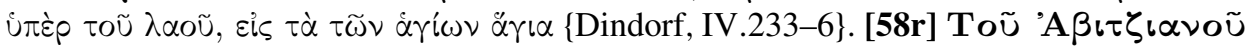

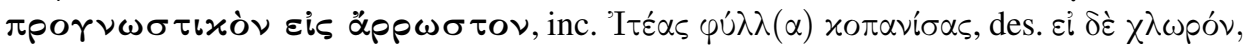

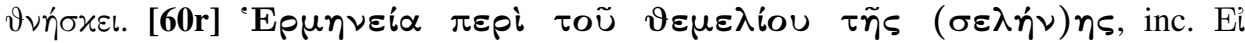

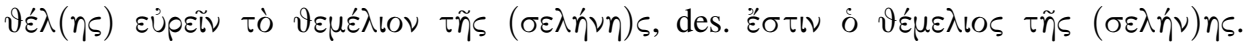

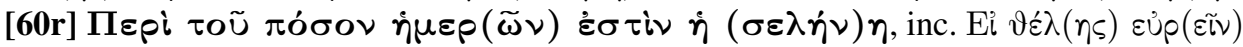

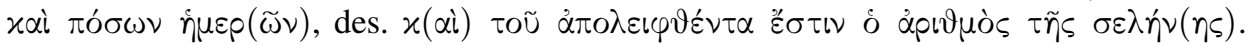

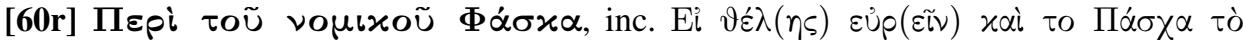

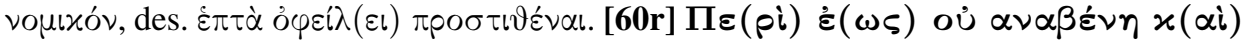

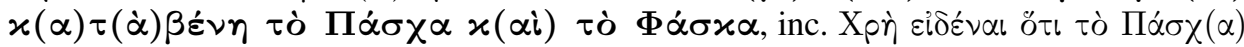

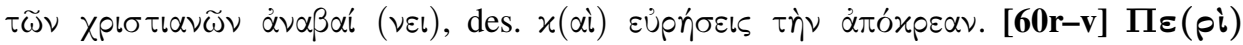

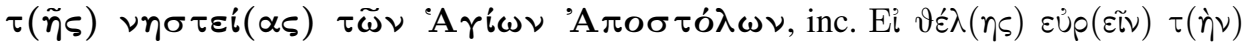

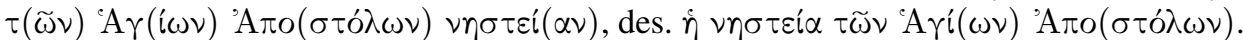

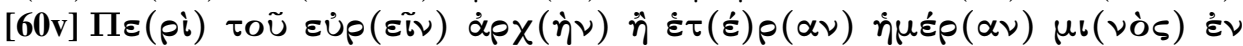

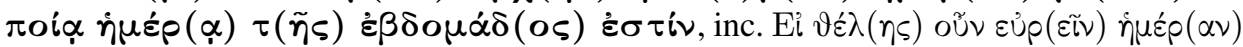

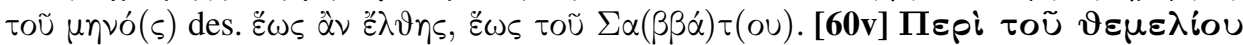

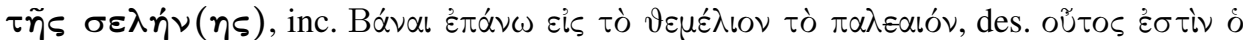

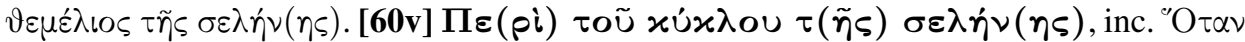

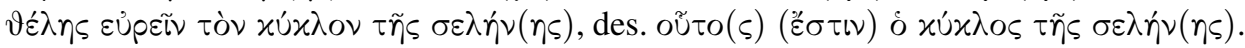

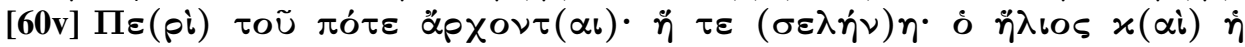

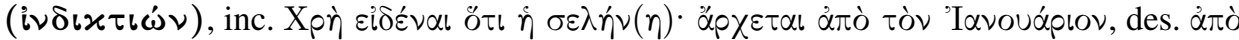

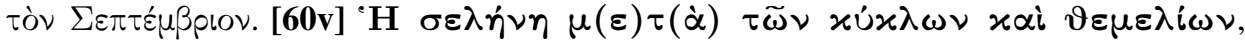

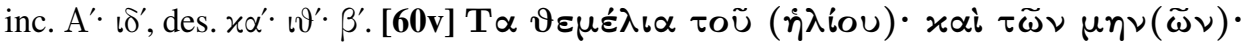

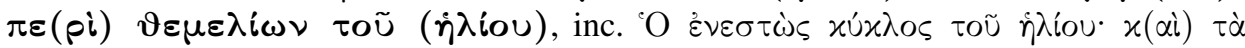

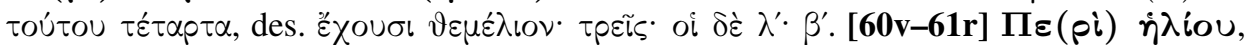

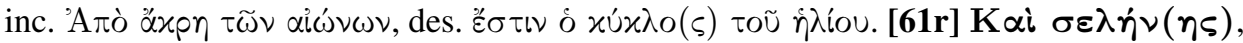

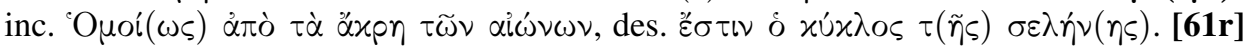

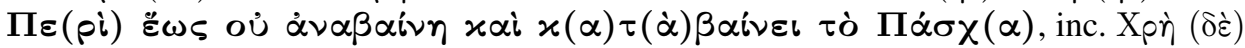

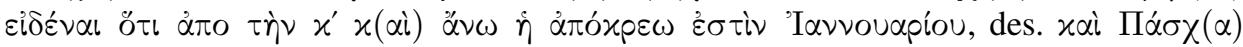

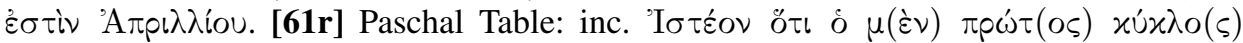

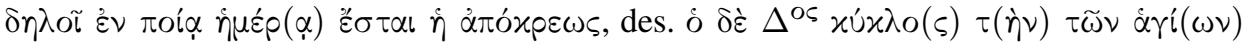

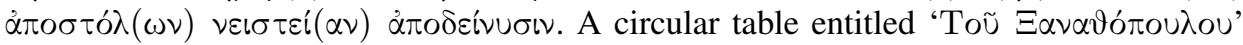




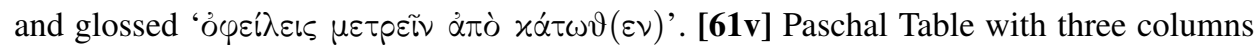

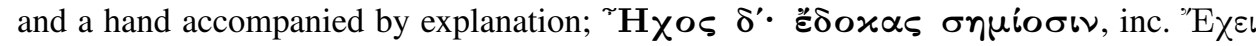

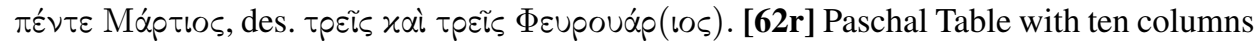

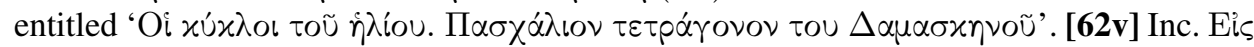

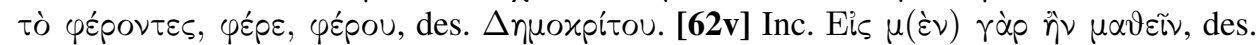

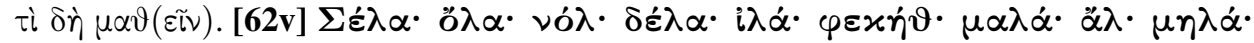

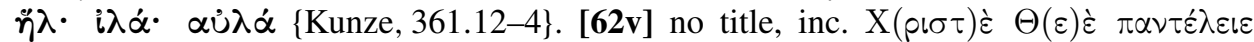

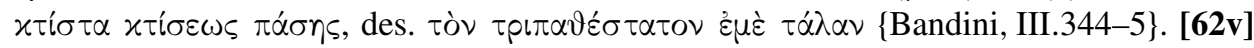

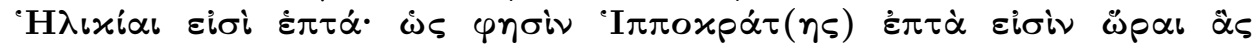

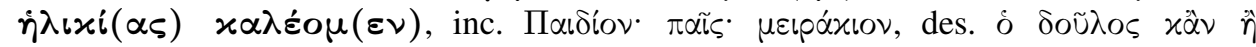

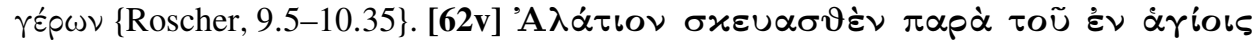

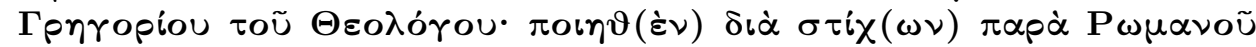

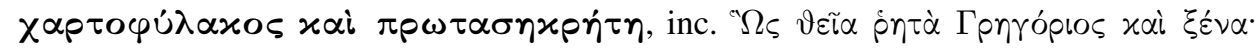

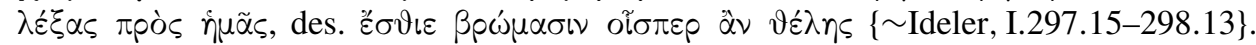

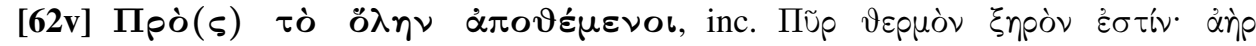

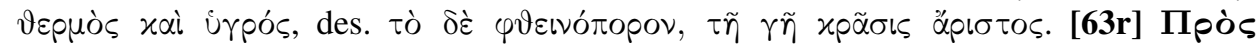

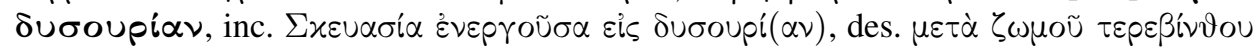

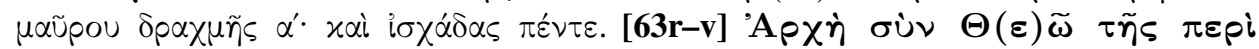

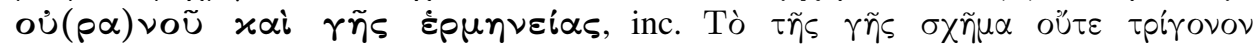

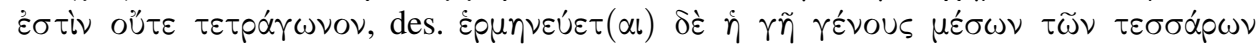

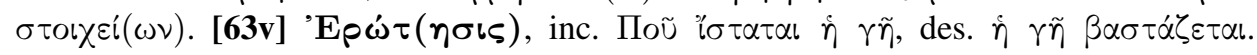

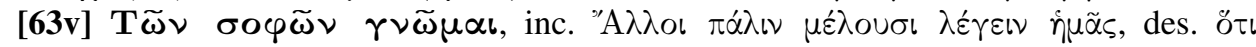

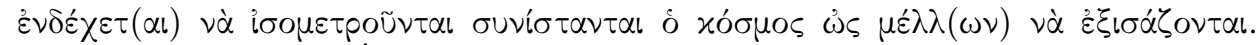

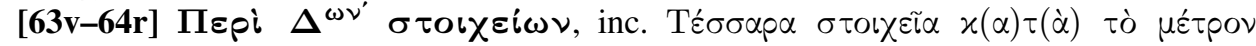

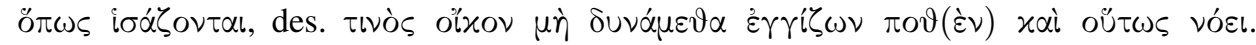

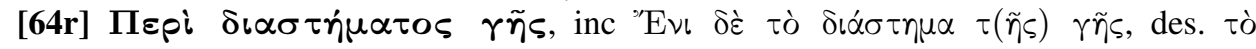

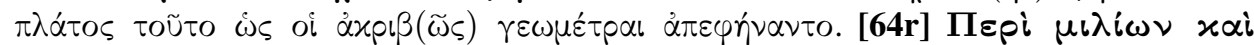

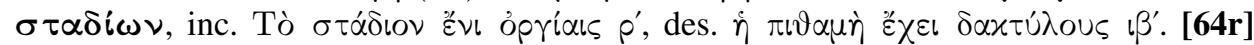

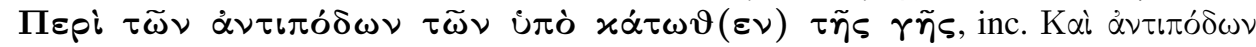

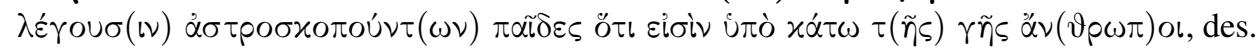

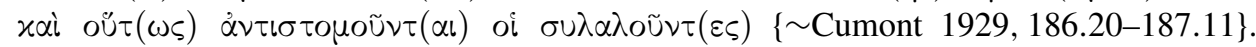

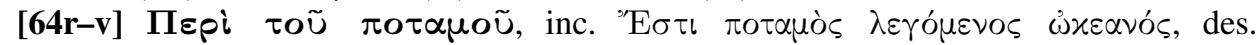

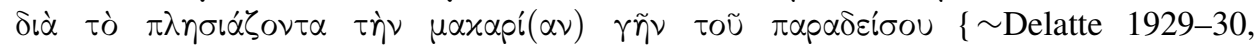

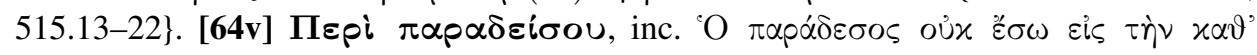

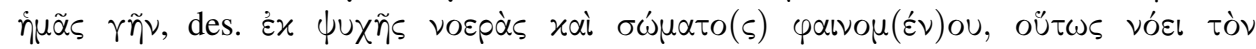

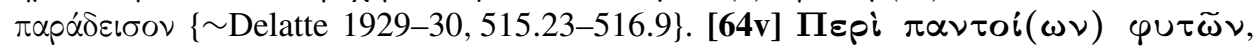

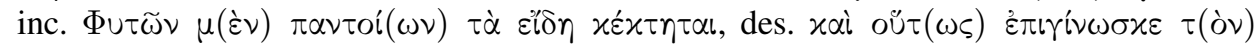

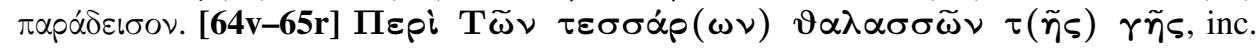

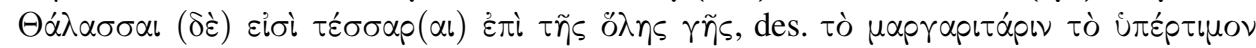

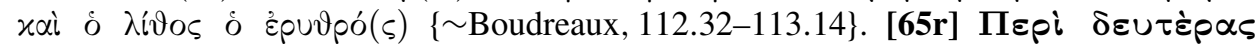

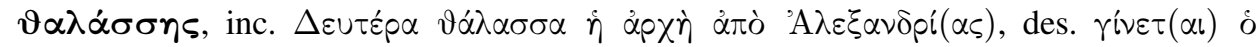

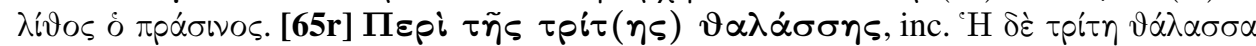

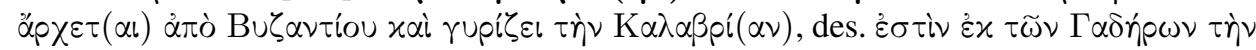

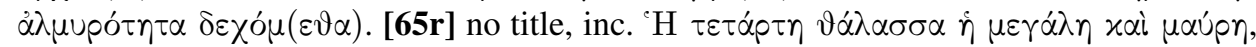

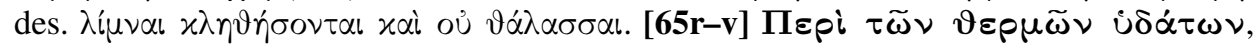

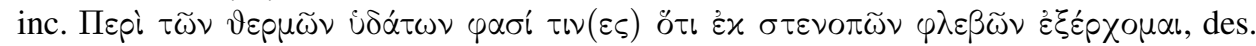

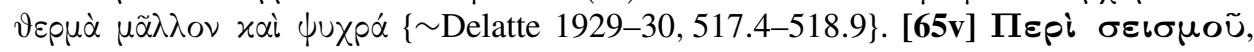




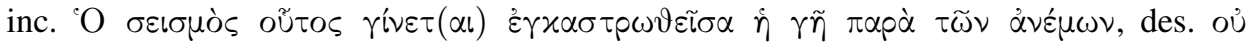

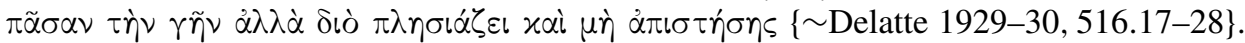

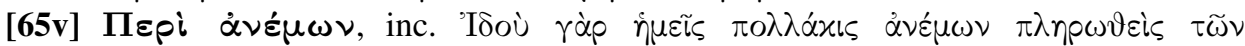

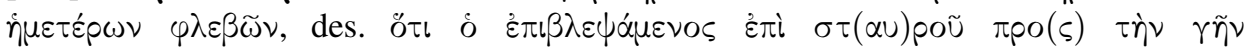

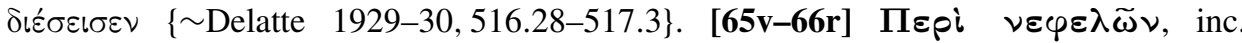

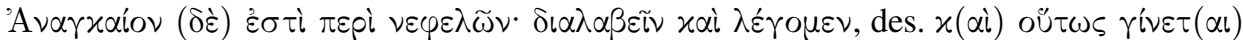

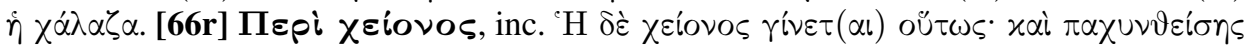

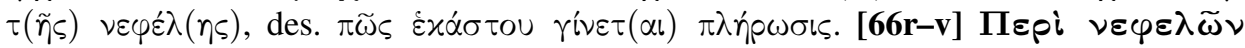

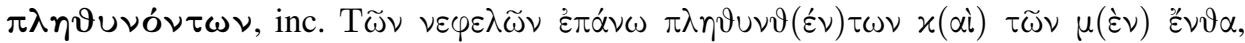

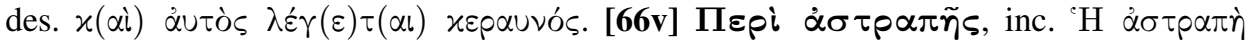

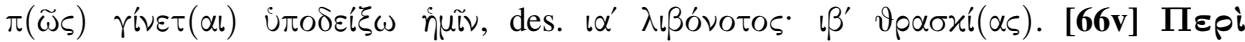

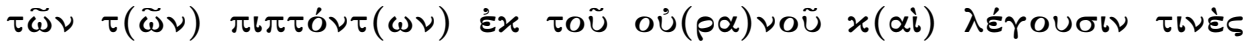

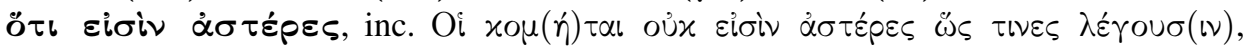

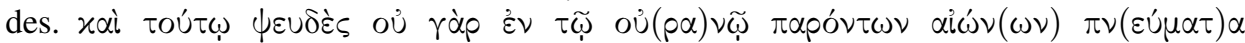

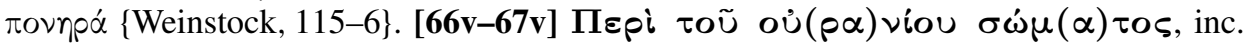

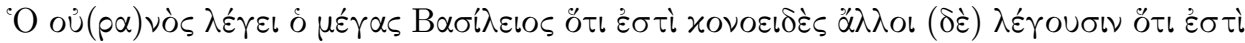

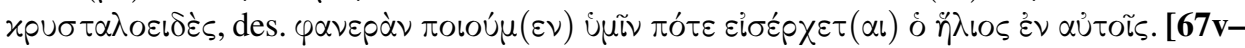

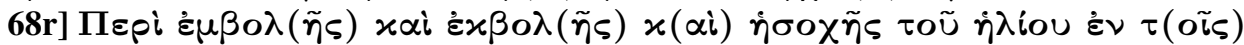

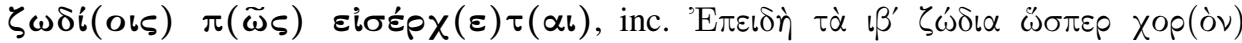

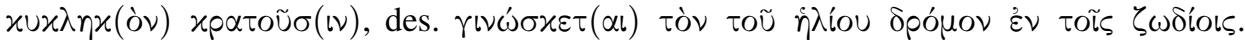

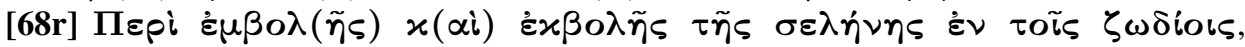

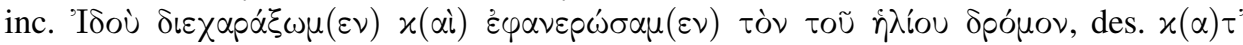

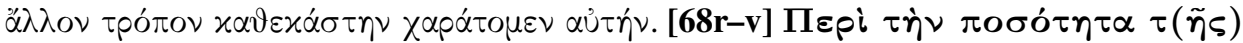

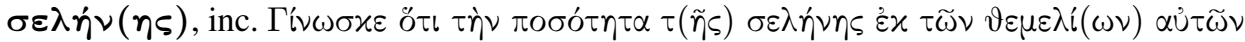

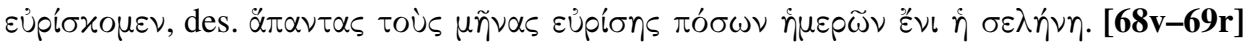

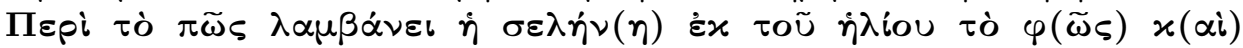

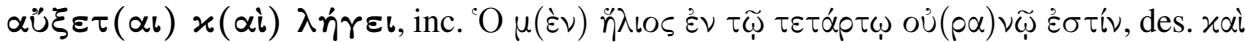

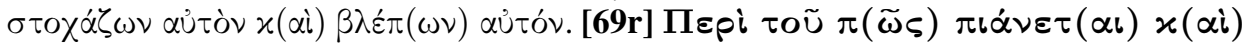

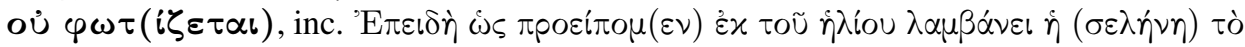

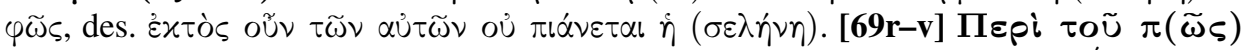

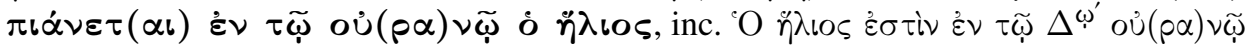

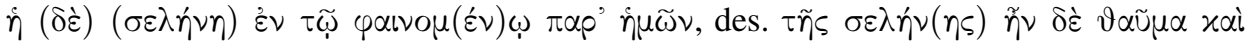

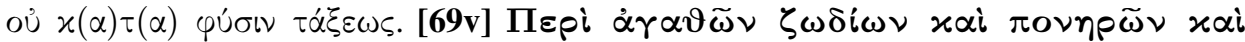

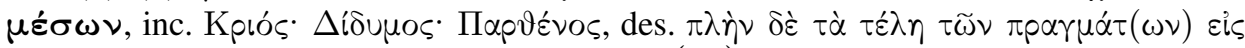

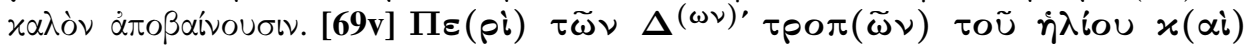

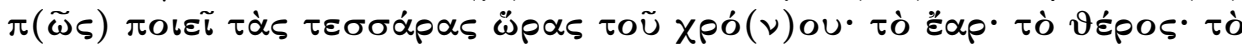

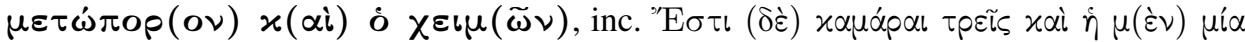

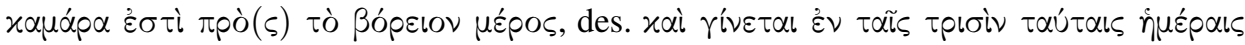

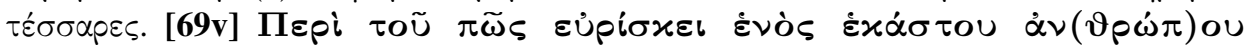

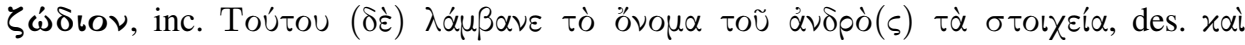

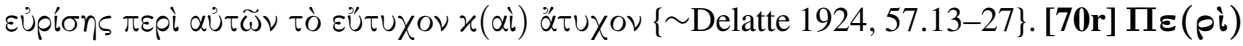

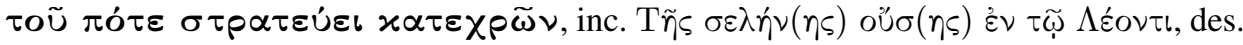

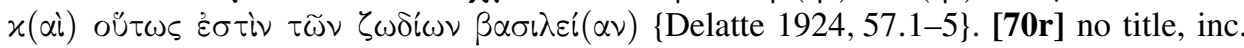

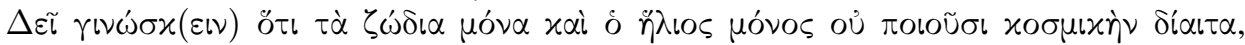

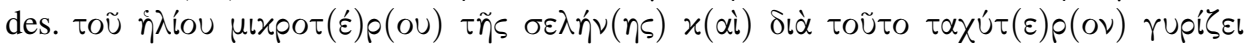

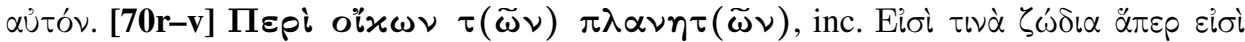

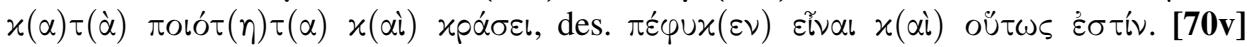




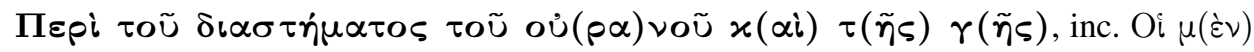

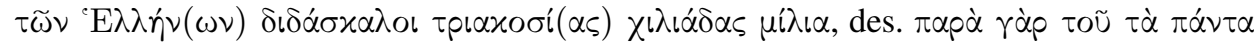

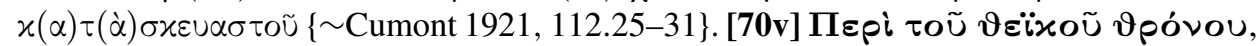

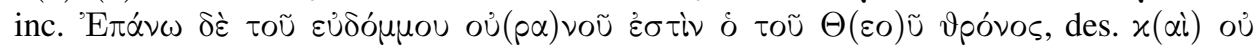

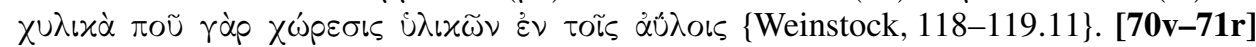

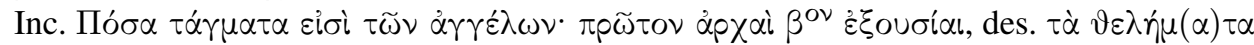

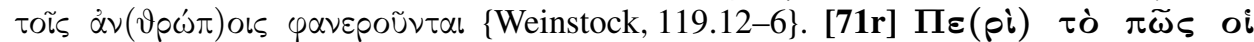

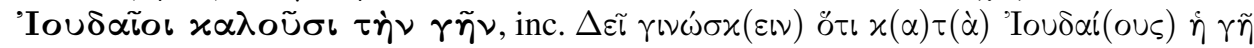

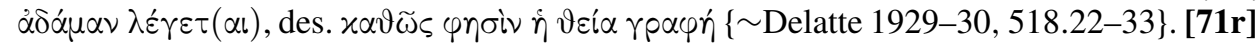

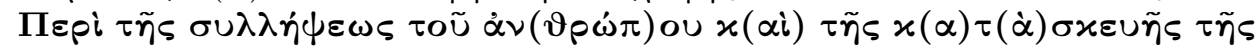

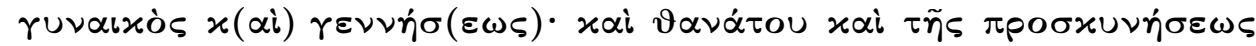

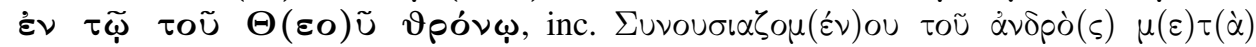

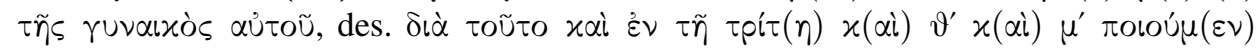

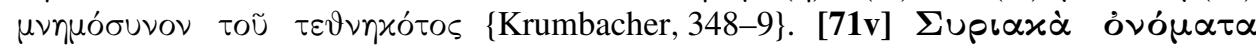

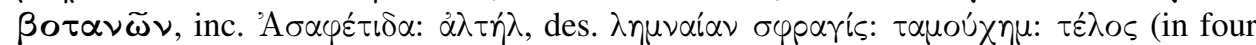

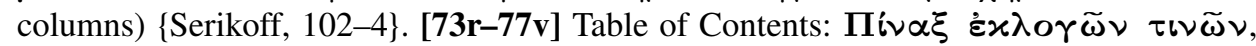

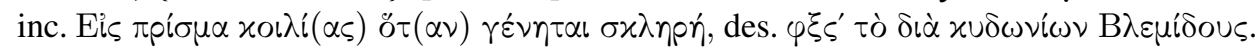

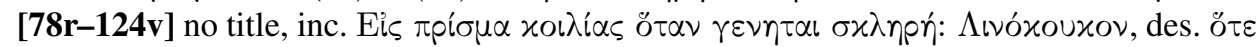

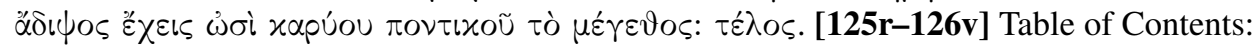

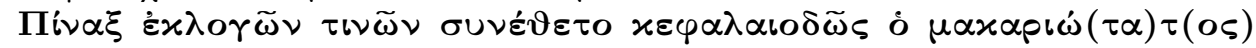

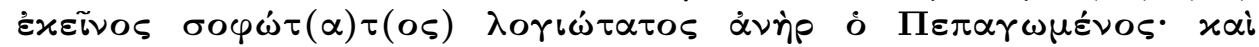

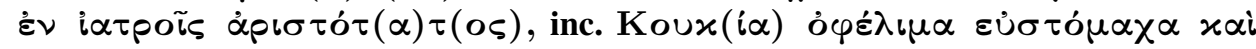

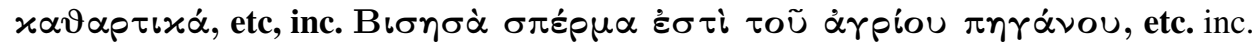

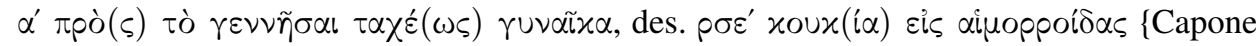

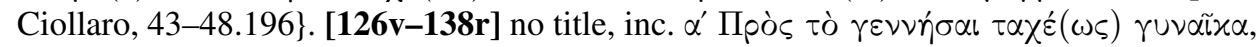

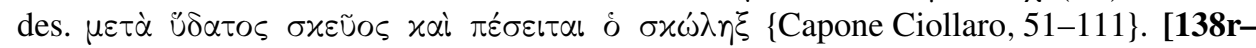

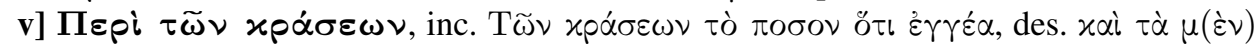

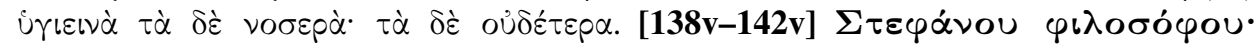

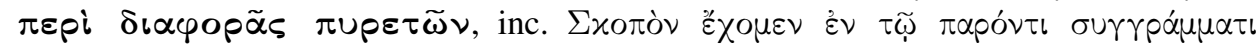

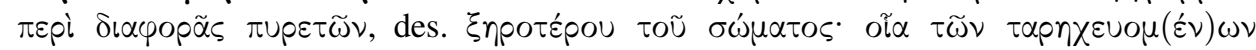

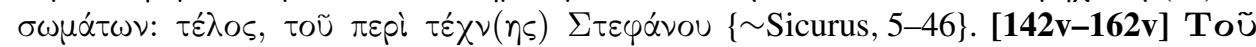

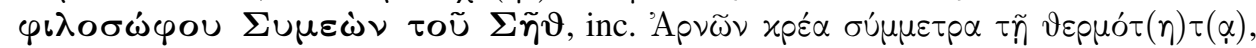

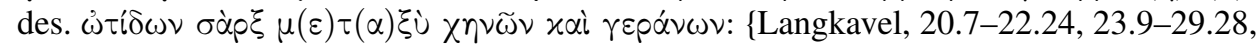
30.11-33.20, 34.1-60.26, 61.8-67.19, 71.14-6, 68.8-71.13, 73.12-80.23, 84.3-85.22, 87.5-88.12, 85.22-86.20, 83.15-84.2, 81.12-83.14, 88.13-99.14, 100.5-12, 99.15-100.4, 100.13-103.7, 104.12-106.8, 107.11-109.10, 111.7-23, 109.11-111.6, 112.1-123.15,

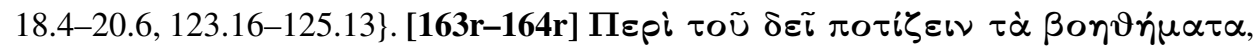

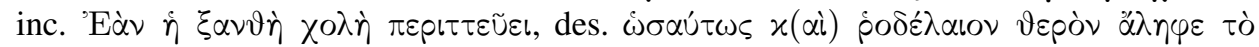

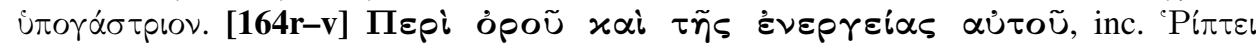

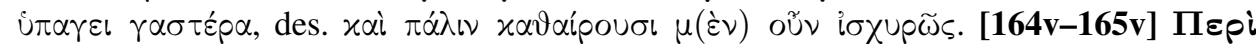

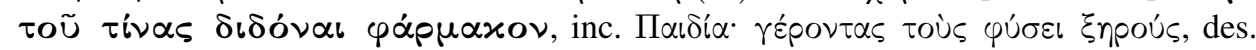

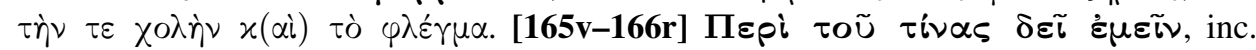

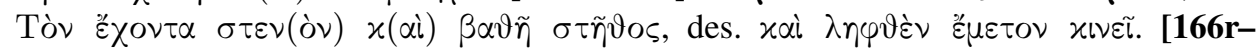

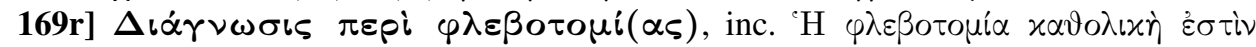

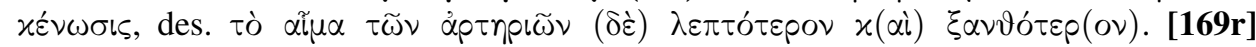

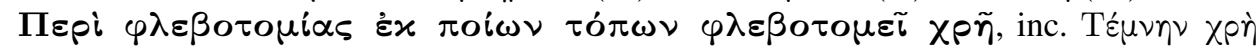

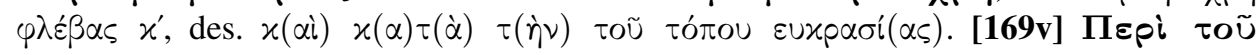




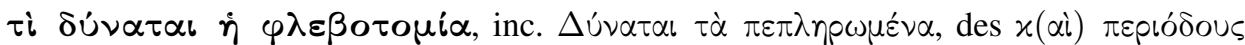

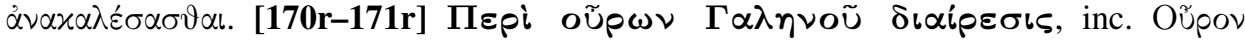

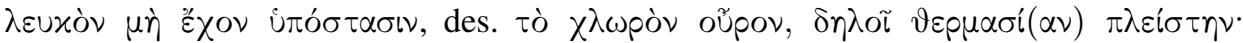

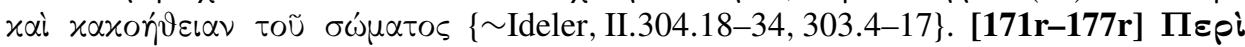

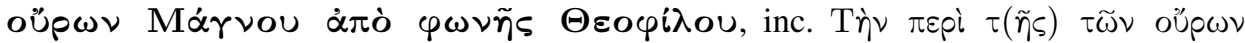

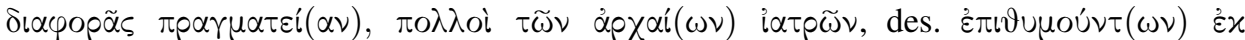

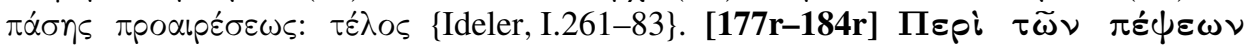

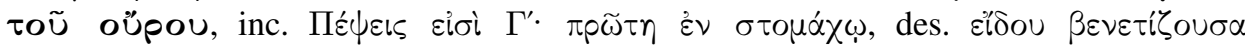

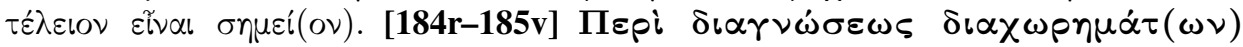

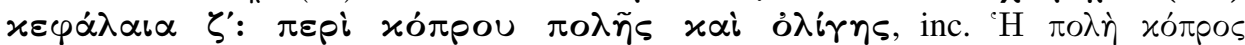

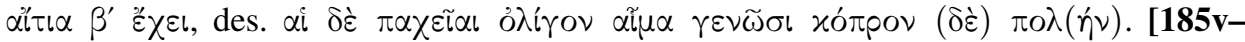

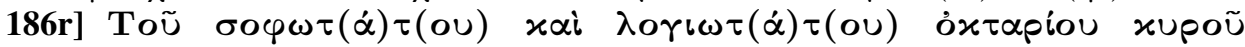

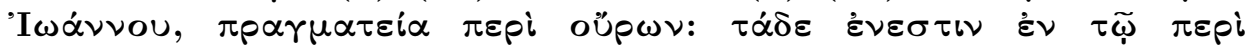

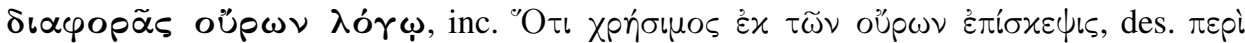

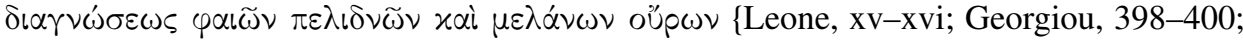

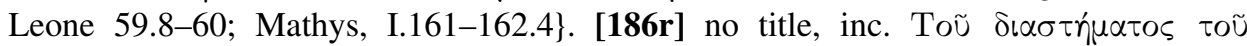

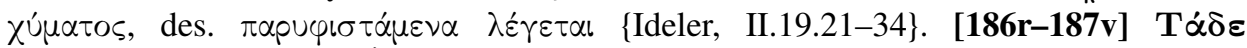

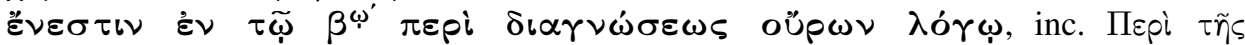

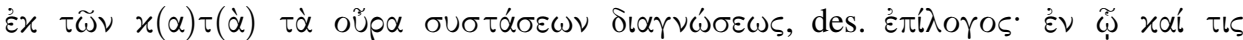

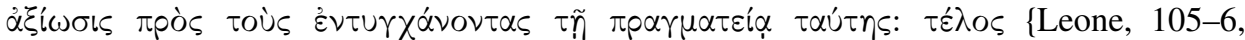
161-2, 229.8-230, 298-9, 354; Mathys, 193-194.16, 232.8-233.11, 280.5-281.18,

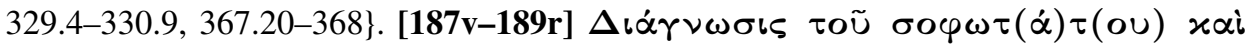

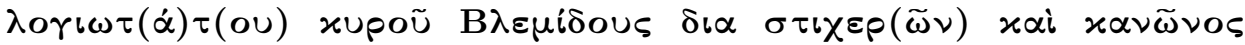

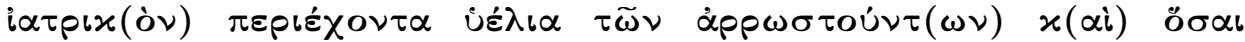

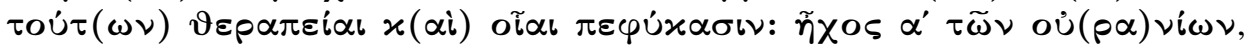

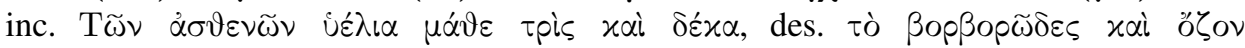

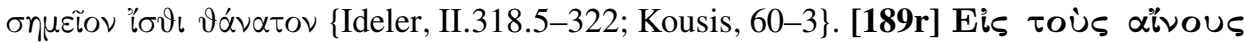

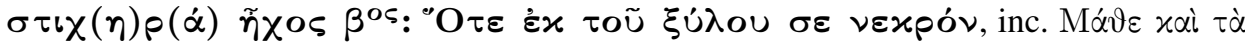

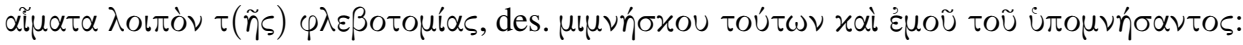

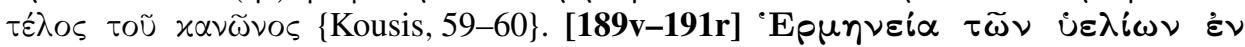

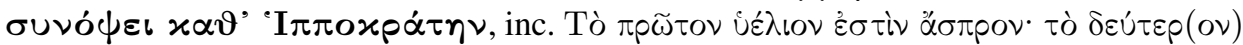

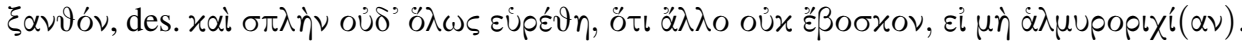

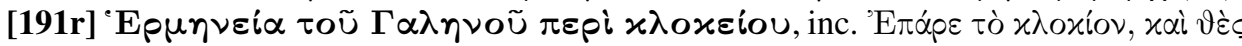

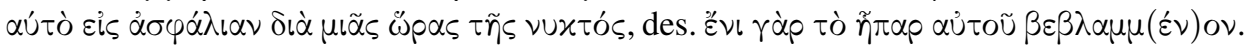

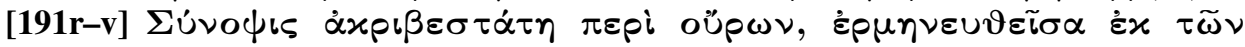

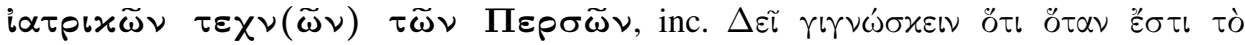

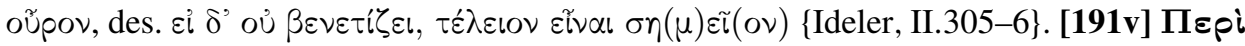

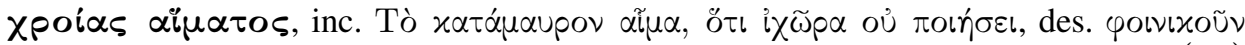

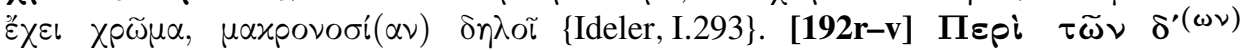

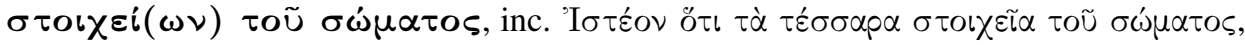

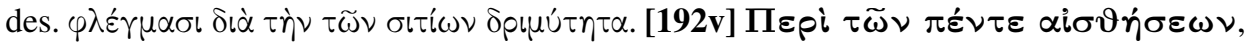

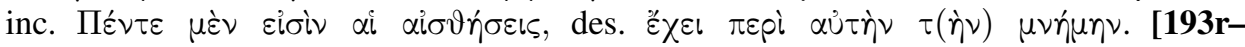

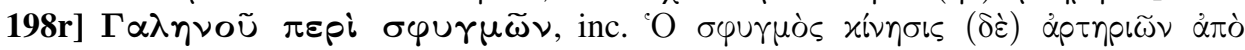

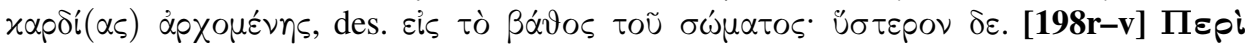

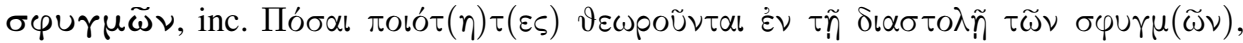

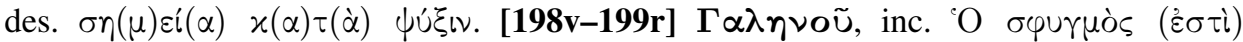

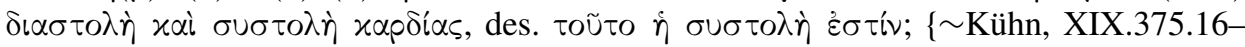




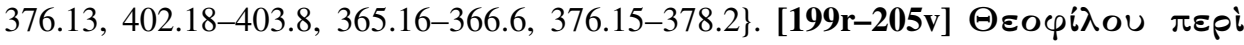

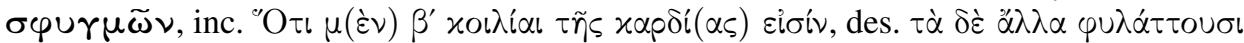

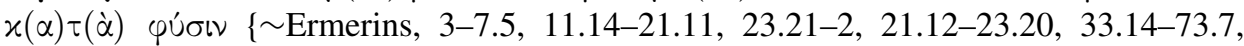

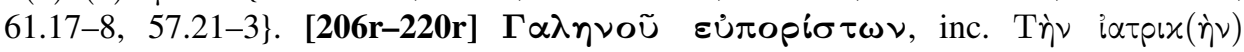

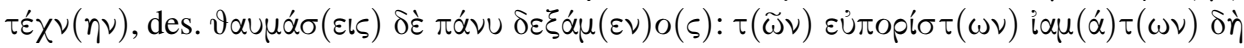

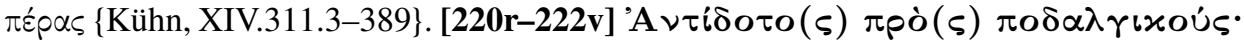

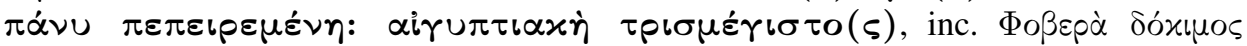

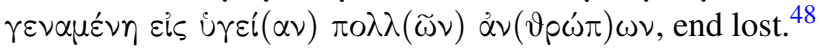

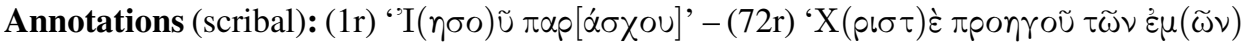

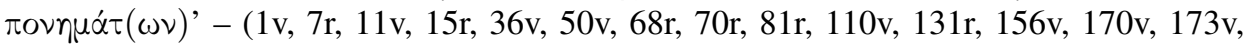
$174 \mathrm{r}, 178 \mathrm{r}, 181 \mathrm{r}, 181 \mathrm{v}, 182 \mathrm{v}, 183 \mathrm{v}, 193 \mathrm{v}, 194 \mathrm{v}, 209 \mathrm{v}, 219 \mathrm{r})$ additions to the main text,

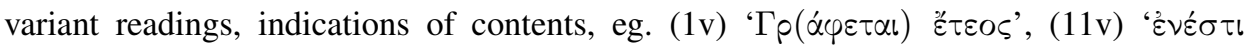
$\tau \alpha \tilde{U} \tau \alpha^{\prime}$.

Illustrations (scribal): $(2 \mathrm{v})$ diagram with numbers $\alpha$ through $\chi$ corresponding to the Hippocratic Aphorism 24 (ch. 2) - (61r) paschal table with concentric circles - (61v) lunar table with two double columns in the sides and a palm with letters inscribed in the phalanxes - (62r) paschal table in 10 columns - (63r) small diagram representing the

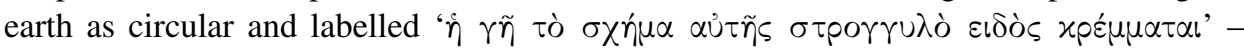
(186r) diagram of a urine vial corresponding to the main text and labelled ' $\alpha \dot{\mu} \iota^{\varsigma}$ ', ' $\nu \varepsilon \varphi \varepsilon$ ' $\lambda \eta$

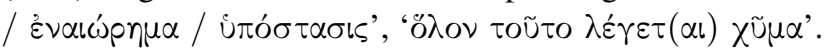

Handwriting: Georgi Parpulov identified the scribe with John (RGK I 204).

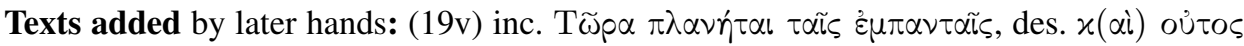

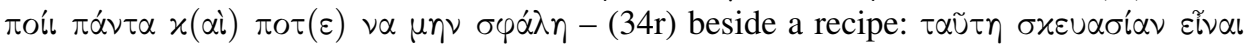

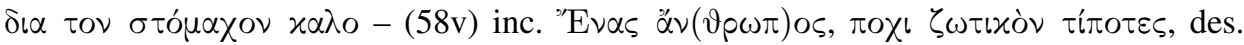

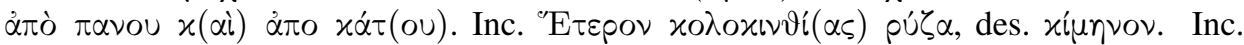

48 Littré, op. cit. (note 38); William Henry Samuel Jones, Hippocrates, with an English Translation, Vol. 4 (Cambridge MA: Harvard University Press, 1931); Bengt Alexanderson, Textkritischer Kommentar zum Hippokratischen Prognostikon und Bemerkungen zu Galens Prognostikonkommentar (Stockholm: Almqvist and Wiksell, 1968); Jacques Jouanna (ed.), Hippocrate: Pronostic (Paris: Les Belles Lettres, 2013); Ideler, op. cit. (note 23); Tartaglia, op. cit. (note 39); Heiberg, op. cit. (note 35); Kühn, op. cit. (note 33); Wilhelm Dindorf, Epiphanii episcopi Constantiae Opera, 5 vols (Leipzig: Weigel, 1859-62); R. Kunze,

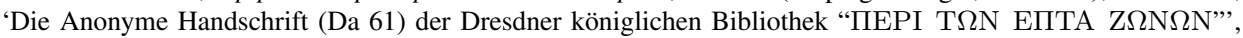
Hermes, 34 (1899), 345-61; Angelo Maria Bandini, Catalogus codicum Graecorum Bibliothecae Mediceae Laurentianae, Vol. 3 (Florence: Typis regiis, 1770); Wilhelm Heinrich Roscher, 'Die hippokratische Schrift von der Siebenzahl', Studien zur Geschichte und Kultur des Altertums, 6, 3-4 (1913), 1-175; Francis Cumont, Catalogus codicum astrologorum Graecorum. VIII. Codices Parisini, pt. 1 (Brussels: Regia Academia Belgica, 1929); Armand Delatte, 'Geographica', Byzantinische Zeitschrift, 30 (1929-30), 511-8; Pierre Boudreaux, Catalogus codicum astrologorum Graecorum. VIII. Codices Parisini, pt. 4 (Brussels: Regia Academia Belgica, 1921); Stefan Weinstock, Catalogus codicum astrologorum Graecorum. IX. Codices Britannici, pt. 2 (Brussels: Regia Academia Belgica, 1953); Armand Delatte, Catalogus codicum astrologorum Graecorum. X. Codices Athenienses (Brussels: Regia Academia Belgica, 1924); Francis Cumont, Catalogus codicum astrologorum Graecorum. VIII. Codices Parisini, pt. 4 (Brussels: Regia Academia Belgica, 1921); Karl Krumbacher, 'Studien zu den Legenden des hl. Theodosius', Sitzungsberichte der philosophisch-philologischen und der historischen Classe der K. B. Akademie der Wissenschaften zu München, (1892), 220-379; Nikolai Serikoff, "Syriac" plant names in a fifteenth century Greek glossary', in Barbara Zipser (ed.), Medical Books in the Byzantine World (Bologna: Eikasmos, 2013), 97-121; Maria Capone Ciollaro, Demetrio Pepagomeno: Prontuario medico (Naples: Bibliopolis, 2003); Sicurus, op. cit. (note 35); August B. Langkavel, Simeonis Sethi Syntagma de alimentorum facultatibus (Leipzig: Teubner, 1868); Leone, op. cit. (note 34); Georgiou, op. cit. (note 34); Mathys, op. cit. (note 34); Kousis, op. cit. (note 46); Ermerins, op. cit. (note 23). 


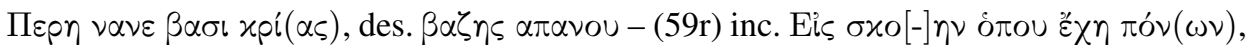

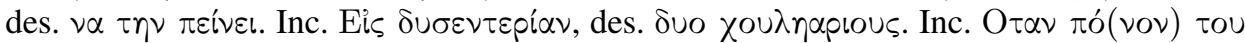

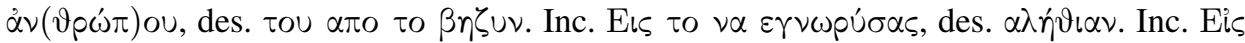

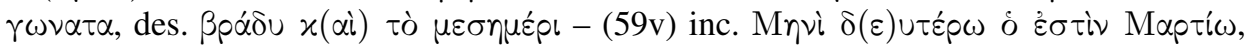

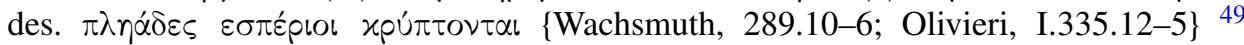

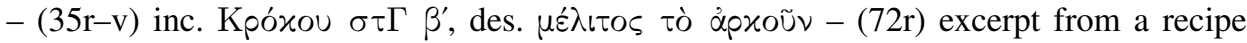

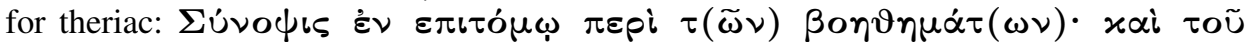

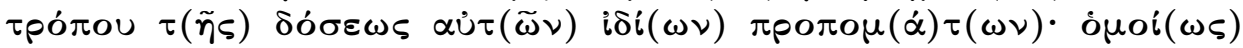

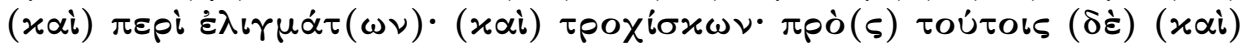

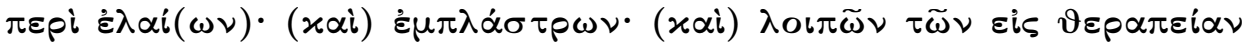

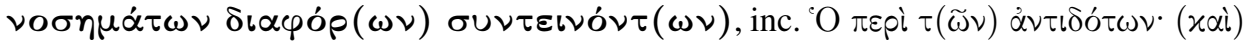

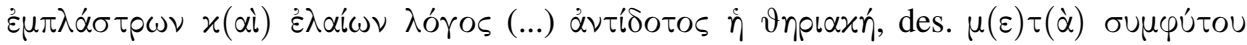

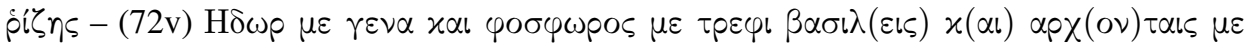

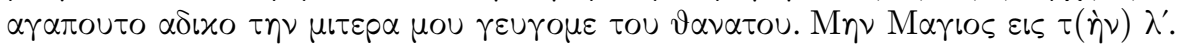

Annotations (non-scribal): (1r, 2v, 4r, 19r, 19v, 20r, 34r, 37v, 45r, 58v, 59r, 59v, 60v, $61 \mathrm{v}, 62 \mathrm{r}, 62 \mathrm{v}, 63 \mathrm{r}, 66 \mathrm{r}, 67 \mathrm{v}, 68 \mathrm{r}, 69 \mathrm{v}, 70 \mathrm{r}, 72 \mathrm{r}, 72 \mathrm{v}, 80 \mathrm{r}, 80 \mathrm{v}, 82 \mathrm{r}, 82 \mathrm{v}, 83 \mathrm{r}, 83 \mathrm{v}, 84 \mathrm{v}$, $85 \mathrm{r}, 85 \mathrm{v}, 86 \mathrm{r}, 88 \mathrm{r}, 89 \mathrm{r}, 89 \mathrm{v}, 90 \mathrm{r}, 90 \mathrm{v}, 91 \mathrm{r}, 91 \mathrm{v}, 92 \mathrm{r}, 94 \mathrm{v}, 95 \mathrm{r}, 99 \mathrm{v}, 100 \mathrm{r}, 101 \mathrm{r}, 104 \mathrm{v}, 105 \mathrm{r}$, $106 \mathrm{r}, 107 \mathrm{r}, 108 \mathrm{r}, 109 \mathrm{r}, 93 \mathrm{v}, 113 \mathrm{v}, 114 \mathrm{v}, 115 \mathrm{r}, 117 \mathrm{v}, 118 \mathrm{r}, 121 \mathrm{v}, 122 \mathrm{r}, 122 \mathrm{v}, 123 \mathrm{r}, 123 \mathrm{v}$, $125 \mathrm{r}, 126 \mathrm{v}, 127 \mathrm{r}, 129 \mathrm{r}, 129 \mathrm{v}, 130 \mathrm{r}, 132 \mathrm{r}, 133 \mathrm{r}, 137 \mathrm{r}, 137 \mathrm{v}, 138 \mathrm{r}, 142 \mathrm{v}, 143 \mathrm{r}, 144 \mathrm{v}, 145 \mathrm{r}$, $145 \mathrm{v}, 146 \mathrm{r}, 146 \mathrm{v}, 147 \mathrm{v}, 149 \mathrm{r}, 149 \mathrm{v}, 150 \mathrm{r}, 152 \mathrm{r}, 153 \mathrm{v}, 155 \mathrm{v}, 156 \mathrm{r}, 162 \mathrm{r}, 163 \mathrm{r}, 165 \mathrm{r}, 168 \mathrm{v}$, $169 \mathrm{r}, 169 \mathrm{v}, 198 \mathrm{v}, 205 \mathrm{v}, 209 \mathrm{v}, 210 \mathrm{r}, 211 \mathrm{v}, 212 \mathrm{r}, 212 \mathrm{v}, 213 \mathrm{r}, 213 \mathrm{v}, 214 \mathrm{r}, 214 \mathrm{v}, 215 \mathrm{v}, 216 \mathrm{r}$,

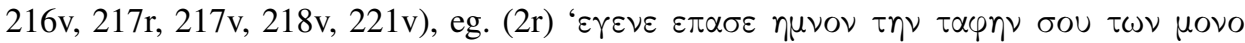

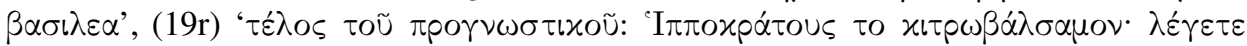

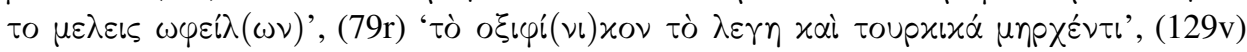
long comment on the effectivenes on a certain recipe of the text, inc. Tò $\varepsilon \mu \pi \lambda \dot{\alpha} \sigma \tau \rho$ o ótov

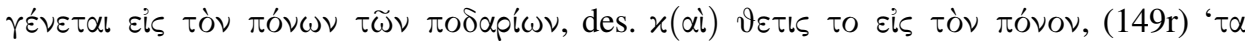

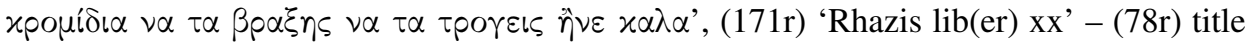

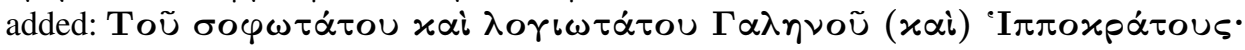

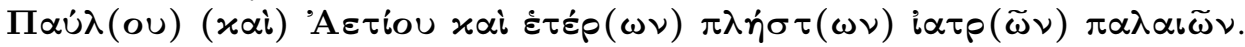

Drawings by later hands: (1r, 1v, 73v, 106v, 160v) obscure figures - (19v) drawing of two

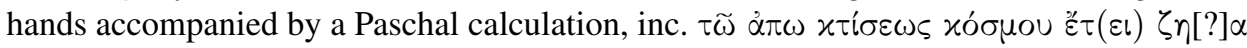

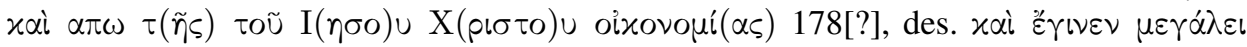
$\dot{\alpha} \pi \eta \lambda \dot{\eta}-(59 r)$ concentric circles.

Paper: folded in $4^{\circ}$; chain distance $33 \mathrm{~mm}$; watermark very similar to Piccard 123360 (arrows), attested in 1455 AD.

Quire signatures: (scribal) Greek numerals $\operatorname{Im} 3 \alpha^{\prime}(27 \mathrm{v}), \operatorname{Im} 1 \beta^{\prime}(28 \mathrm{r})$ and $\gamma^{\prime}(36 \mathrm{r}), \operatorname{Ie} 1 \gamma^{\prime}$ (70r, 73r) through $\sigma \tau^{\text {ov }}(97 \mathrm{r})$, Ie $3 \delta^{\prime}(88 \mathrm{v})$, Ii3 $\varepsilon^{\prime}(96 \mathrm{v})$, Ie $1 \alpha^{\prime}(206 \mathrm{r})$.

Quires: 8 (f. 8), 2 (f. 10), 8 (f. 18), 1 (f. 19), $3 \times 8$ (f. 43), 2 (f. 45), 6 (f. 51 ), 8 (f. 59), 12 (f. 71$), 1$ (f. 72 ), $5 \times 8$ (f. 112), $2 \times 4$ (f. 120), $10 \times 8$ (f. 201), 4 (f. 205), $2 \times 8$ (f. 221 ).

Binding: corio russico, same tooling as Wellcome MS.MSL.1. - Flyleaves: chain distance $25 \mathrm{~mm}$, no watermarks.

49 Curt Wachsmuth, Ioannis Laurentii Lydi Liber de ostentis, et calendaria Graeca omnia (Leipzig: Teubner, 1897); Olivieri, op. cit. (note 35). 


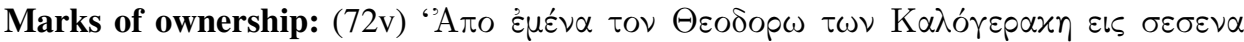

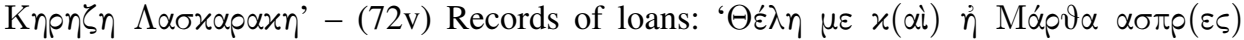

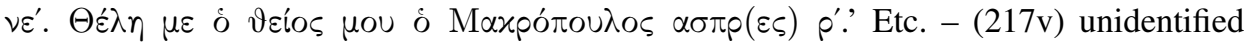

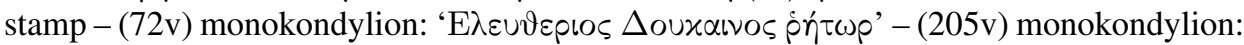

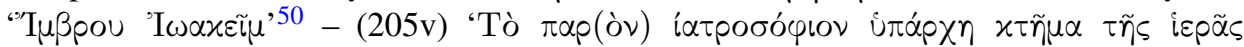

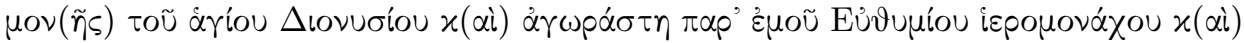

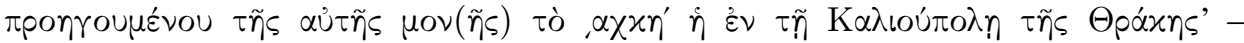

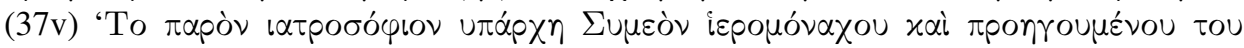

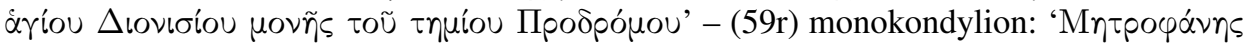

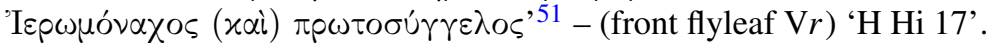

Provenance: bought in Gallipoli by Euthymius, former abbot of the Dionysiou Monastery, Mount Athos, in 1628 - Symeon, former abbot of the Dionysiou Monastery, Mount Athos - Anthony Askew (1722-74), London; [his sale, G. Leigh and J. Sotheby, London, 15 March 1785, lot 577]; purchased by James Sims (1741-1820), London, in 1785; purchased by the London Medical Society in 1802; purchased by the Wellcome Library in 1984.

Bibliography: Bibl. Askev., 35 (no. 577); Cat. Med. Soc. 1803, 96; Cat. Med. Soc. 1829, 155, 286; Daremberg, 159-162; Nias, liii-liv; Diels, I.5, 13, 48, 100, 115, 125, 128, 131, 133, II.7, 79, 80, 98, 101-102, 109; Dawson, 68-72; Weinstock, op. cit. (note 48), 33-38 (no. 56); Alexanderson, op. cit. (note 48), 76; Tartaglia, op. cit. (note 39), 548; Capone Ciollaro, op. cit. (note 48), 12-20; Nutton and Zipser, 261; Touwaide, 538-9; Georgiou, op. cit. (note 34), 253; Jouanna, op. cit. (note 48), 1xxxv, xci; Serikoff, op. cit. (note 48), 97-121.

Alain Touwaide, 'Lexica medico-botanica byzantina: Prolégomènes à une étude', in Lois

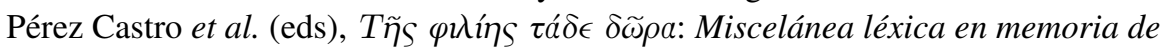
Conchita Serrano (Madrid: Consejo Superior de Investigaciones Científicas, 1999), 211-228: 215, 227.

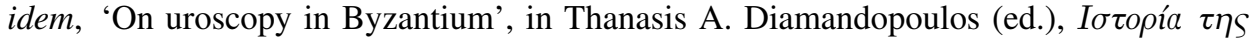

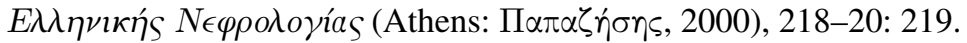

idem, 'Arabic urology in Byzantium', Journal of Nephrology, 17 (2004), 583-9: 585.

Maria Capone Ciollaro, 'Per l'edizione delle Eclogai di Demetrio Pepagomeno', in Antonio Garzya and Jacques Jouanna (eds), Trasmissione e ecdotica dei testi medici greci: Atti del IV Convegno Internazionale, Parigi 17-19 maggio 2001 (Naples: D'Auria, 2003), 40-52: 40.

\section{MS.MSL.62 (olim HH i 23 / We 32a)}

Italy, ca. 1520-40 AD (from watermarks).

Paper, $223 \times 163$, iv $+157+\mathrm{i}($ foliated $1-55,56-88,89-155)$

\footnotetext{
50 Probably the metropolitan of Imbros attested ca. 1548-80: Martin Crusius, Turcograecia (Basel: Leonardus Ostenius, 1584), 287, 318.

51 On Euthymios, Symeon (d. 1652), and Metrophanes (d. 1655): Sotiris K. Kadas, Tà $\sigma \eta \mu \epsilon \hat{\omega} \mu a \tau a$ $\tau \widetilde{\omega} \nu$

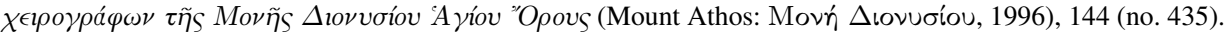


[1r-31r] Aretaeus of Cappadocia, On the Causes and Symptoms of Acute Diseases (TLG 0719.001). [31v-78v] Idem, On the Causes and Symptoms of Chronic Diseases (TLG 0719.002). [78v-122v] Idem, On the Cure of Acute Diseases (TLG 0719.003). [127r-139v] Rufus of Ephesus, On the Nomenclature of the Human Body (TLG 0564.003). [139v-143r] Ps.-Rufus of Ephesus, On the Parts of the Human Body (TLG 0721.001). [143r-150r] Rufus of Ephesus, On the Parts of the Human Body (TLG 0564.004). [150r-152v] Idem, On Bones (TLG 0564.005).

Note: This MS consists of two distinct parts, described below separately as $\mathbf{A}$ and $\mathbf{B}$.

A. Linn. 20 [132 $\times 84], 20$ D 1 .

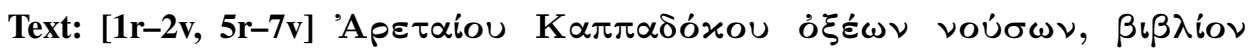

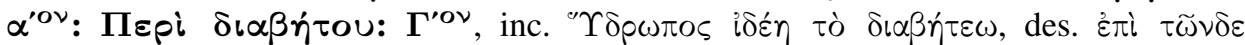

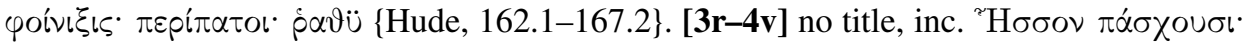

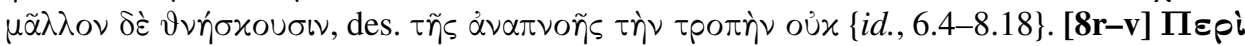
$\tau \varepsilon \tau \dot{\nu} \nu o u$, inc. $\Sigma \pi \alpha \sigma \mu o \grave{~ o i ~ \tau \varepsilon ́ \tau \alpha \nu o l, ~ d e s . ~ \nu \varepsilon ́ o เ ~ \delta \varepsilon ̀ ~ \tau o u \tau \varepsilon ́ \omega \nu ~\{i d ., ~ 5.14-6.4\} . ~[9 r-13 v] ~ n o ~}$

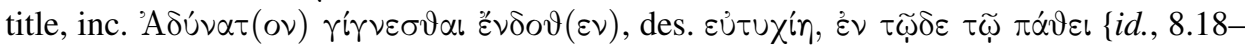

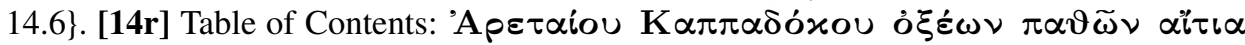

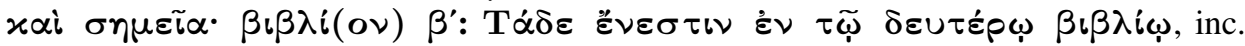

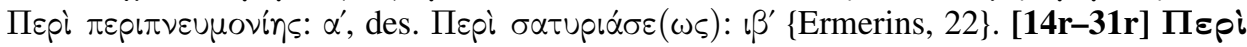

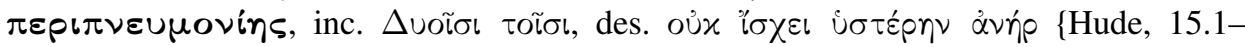

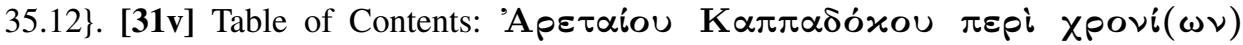

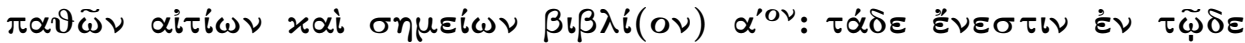

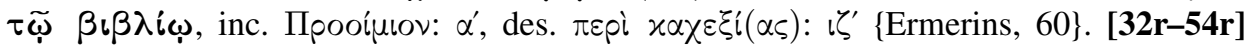

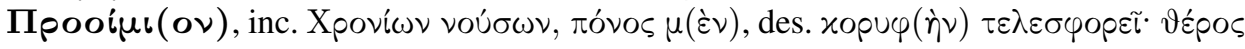

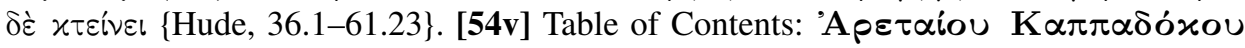

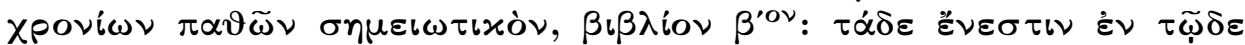

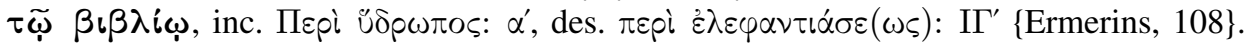

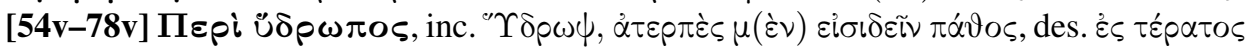

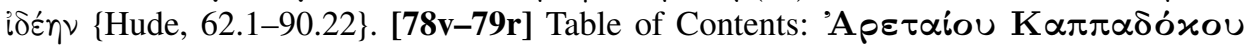

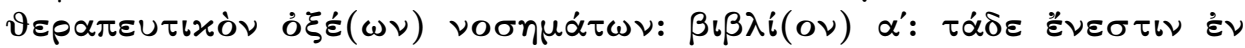

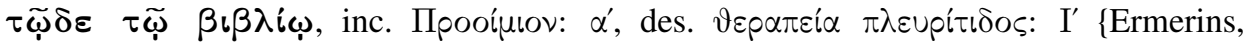

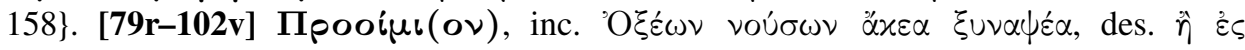

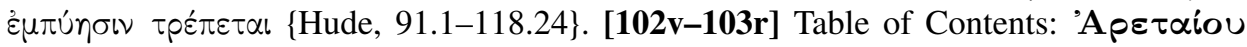

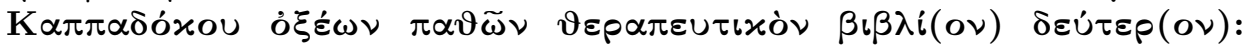

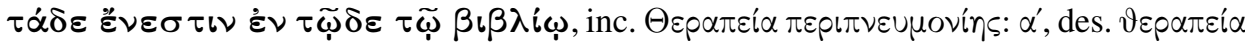

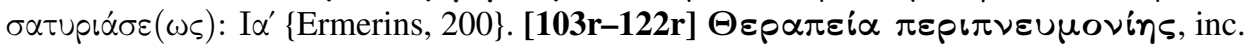

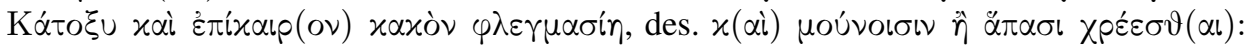

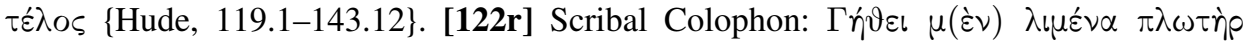

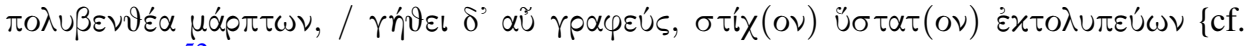
Vassis, 118\}. ${ }^{52}$

Blank pages: $122 \mathrm{v}-126 \mathrm{v}$.

\footnotetext{
52 Franz Zacharias Ermerins, Aretaei Cappadocis quae supersunt (Utrecht: Kemink, 1847); Karl Hude, Aretaeus, 2nd edn (Berlin: Academia Scientiarum, 1958) [CMG, II]; Ioannis Vassis, Initia carminum Byzantinorum (Berlin: De Gruyter, 2005).
} 
Annotations (scribal): (61r, 66r, 73r, 79v, 83r, 83v, 84v, 87r, 88r, 88v, 90r, 91r, 99v, 101v, 107r, 108r, 109r, 109v, 110r, 110v, 111r, 111v, 115r, 115v, 117r, 118r, 120r, 121v) additions to the text, variant readings or short explanations in the form of synonyms, eg. (61r) i $\sigma(\omega \varsigma)$

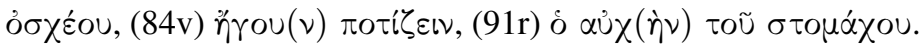

Handwriting: unidentified Humanist hand.

Paper: folded in $4^{\circ}$; chain distance $30 \mathrm{~mm}$; watermark similar to Piccard 122768 (ladder), attested in 1528 .

Catchwords (scribal): vertical, Ii3.

Quire signatures (scribal): Greek numerals Iel $\alpha^{\prime}(1 \mathrm{r})$ through $\iota \tau^{\prime}(119 \mathrm{r})$.

B.

Linn. $22[140 \times 91]$, unclear ruling.

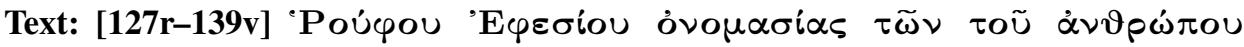

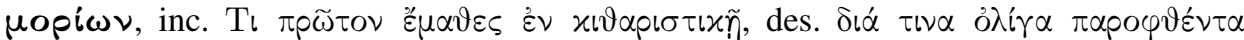

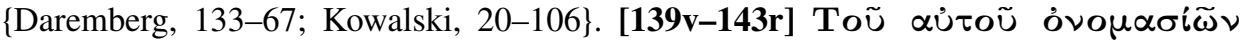

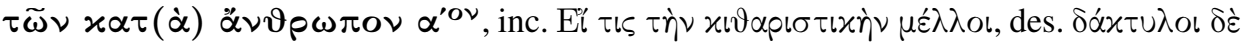

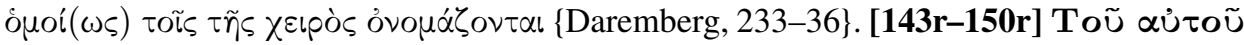

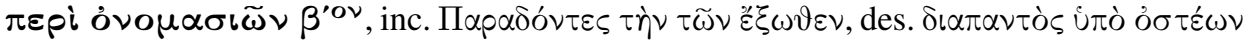

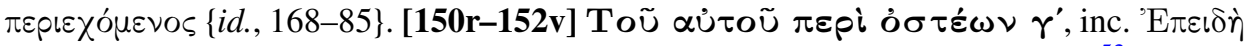

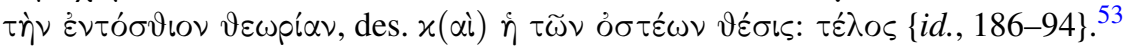

Blank pages: $153 \mathrm{r}-155 \mathrm{v}$.

Annotations (scribal): (128r, 128v, 129r, 129v, 131r, 133r, 133v, 135r, 138v, 139v, 150r) additions to the text or variant readings, eg. (133r) ' $\chi \varepsilon\llcorner\rho \widetilde{\omega \nu}$ ', (150r) ' $\Gamma \rho(\alpha \varphi \varphi \varepsilon \tau) \alpha l \quad \varkappa \alpha \tau(\alpha)$ '.

Handwriting: unidentified Humanist hand.

Paper: folded in $4^{\circ}$; chain distance $32 \mathrm{~mm}$; watermark very similar to Piccard 122766 (ladder), attested in 1533.

Catchwords (scribal): vertical, Ii3.

Quire signatures: (probably scribal) partly lost in trimming: Greek numbers Ie1, eg. $\delta^{\prime}$ (150r).

\section{A. and B.}

Quires: 1 (i), 2 (ii-iii), 1 (iv), 8 (ff. 3 and 4 misplaced; original order 1, 2, 5, 6, 7, 8, 3, 4), $15 \times 8$ (f. 126), 7 (f. 133, no text lost), $2 \times 8$ (f. 149), 6 (f. 155), 1 (i).

Binding: corio russico. - Flyleaves: (i and i) machine-made paper; (ii-iii, front pastedown) chain distance $24 \mathrm{~mm}$, no watermark - (iv) chain distance $40 \mathrm{~mm}$, watermark generally similar to Briquet 5252 (croissant), attested in 1530-2 - (back pastedown) chain distance $30 \mathrm{~mm}$, no watermark.

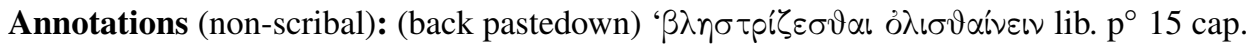
/ deest [?] cap. 2'.

53 Daremberg, op. cit. (note 33); Georg Kowalski, 'Rufi Ephesii De corporis humani pertium appellationibus' (unpublished PhD thesis: University of Göttingen, 1960). 
Marks of ownership: (front flyleaf II $r$ ) 'Ex Bibliotheca Askeviana / P. ii. Art. 551. / J. Sims' - (1r) 'A. Askew M. D.' - (1r) '3.28'.

Provenance: Anthony Askew (1722-74), London; [his sale, G. Leigh and J. Sotheby, London, 15 March 1785, lot 551]; purchased by James Sims (1741-1820), London, in 1785; purchased by the London Medical Society in 1802; purchased by the Wellcome Library in 1984.

Bibliography: Bibl. Askev., 34 (no. 551); Cat. Med. Soc. 1803, 10; Cat. Med. Soc. 1829, 13; Daremberg, 162-3; Nias, liv; Diels, II.17-18, 89; Dawson, 74; Nutton, 23-5; Nutton and Zipser, 261; Touwaide, 538-9.

Francis Adams (ed.), The Extant Works of Aretaeus, the Cappadocian (London: Sydenham Society, 1856), xvii.

Carmelo Cutolo, 'Sulla tradizione manoscritta di Areteo di Cappadocia', Galenos, 6 (2012), 25-47: 26.

\section{MS.MSL.109 (olim MM c 7 / Wf 7)}

Italy, ca. $1510 \mathrm{AD}$ (from watermarks).

Paper, $319 \times 216$, iv $+316+$ iii (paginated 1 [f. 1v]-163, then foliated 82-318), linn. 30 [ca. $235 \times 125]$, unclear ruling.

Aetios of Amida, Tetrabiblon, Books $9^{54}$ (TLG 0718.009), 10 $0^{55}, 11^{56}$ (TLG 0718.011), $12^{57}$ (TLG 0718.012), $13^{58}$ (TLG 0718.013), 14 $4^{59}, 15^{60}$ (TLG 0718.013).

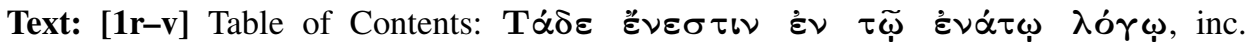

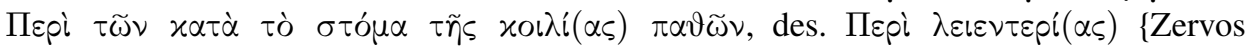

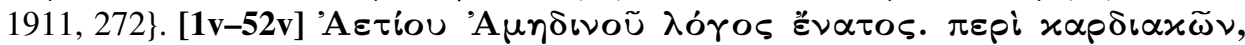

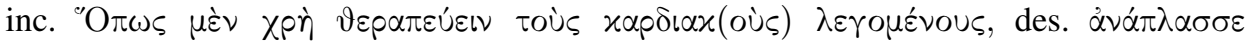

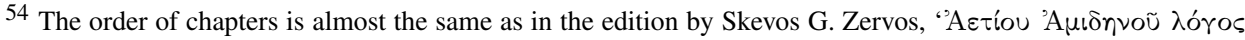
हैข

55 The book has no Greek edition and has only been printed in Latin translations: Giovanni Battista da Monte (trans.), Aetii Amideni Libri sexdecim nunc primum latinitate donati, 3 vols (Venice: Lucas Antonius Junta, 1535); Janus Cornarius (trans.), Aetii medici graeci contractae ex veteribus medicinae tetrabiblos (Lyons: Godefridus et Marcellus Beringi fratres, 1549). Books 8-13 are identical in these two; here, I provide reference to both. The order of chapters in the manuscript sometimes differs from that of the printed Latin translations.

56 The order of chapters differs considerably from that in Daremberg, op. cit. (note 33), 85-126: 568-81.

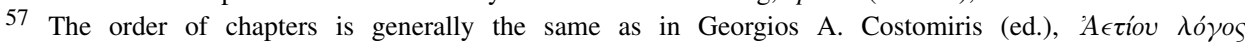
$\delta \omega \delta$ ́́ $\kappa a \tau o S$ (Paris: Klincksieck, 1892); some chapters are supplemented here with further recipes, while the last two chapters printed in Costomiris are missing.

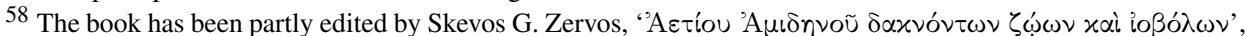

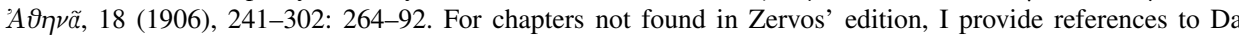
Monte and Cornarius, op. cit. (note 55). Sometimes the text differs considerably from the printed edition and the Latin translations; in one case, an entire chapter is printed neither in Zervos nor in the Latin translations, viz.

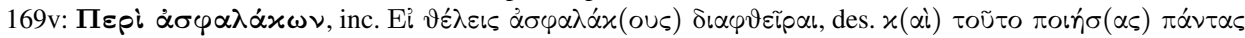

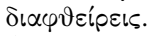

59 The book has no Greek edition and is only available in Latin translations by Da Monte and Cornarius, op. cit. (note 55); here, I provide reference to both. The order of chapters in the manuscript sometimes differs from that in the printed Latin translations.

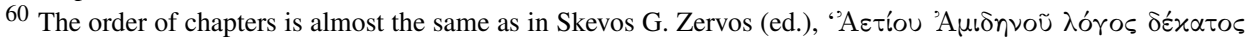

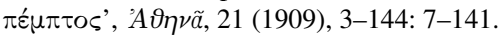




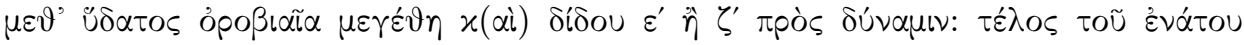

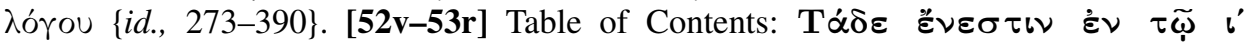

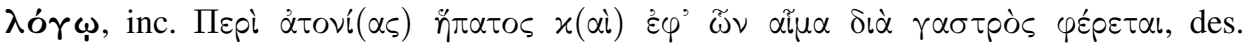

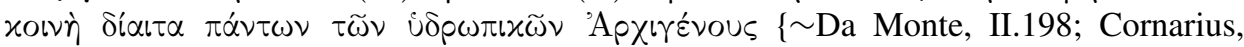

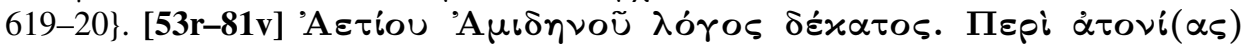

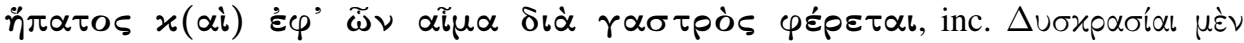

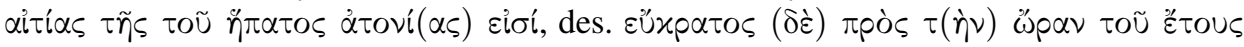

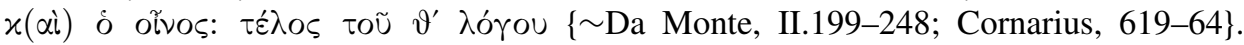

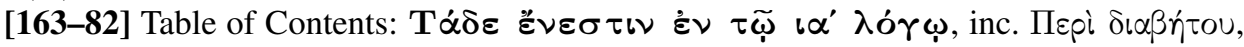

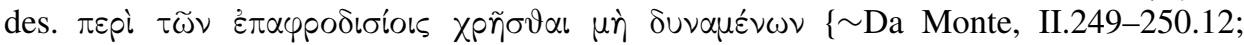

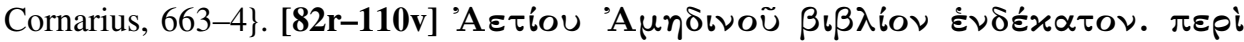

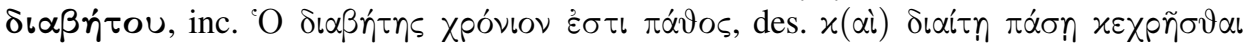

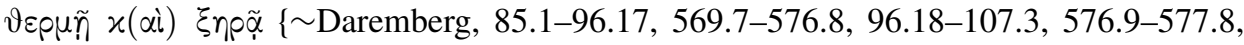
$108.1-109.8$, 577.9-40, 109.9-117.5, 578.2-580.18, 117.6-119.22, 107.4-26, 119.23-

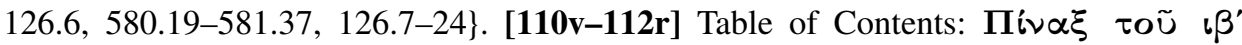

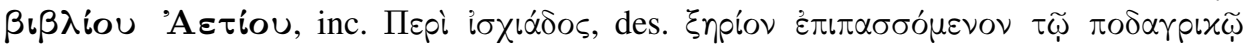

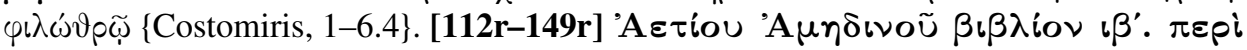

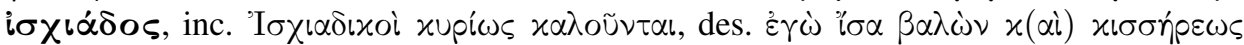

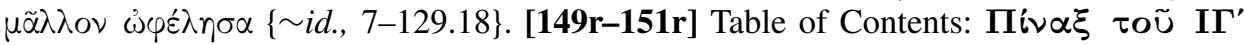

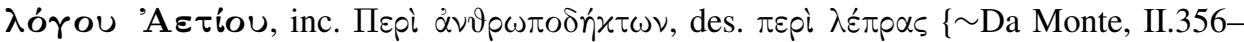

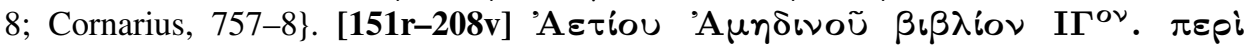

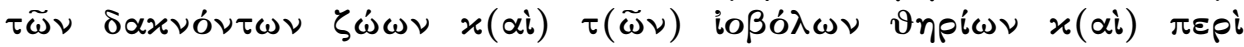

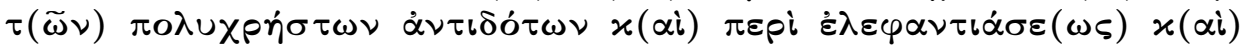

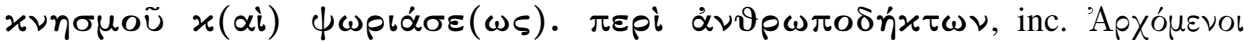

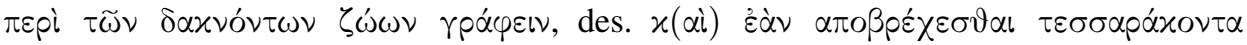

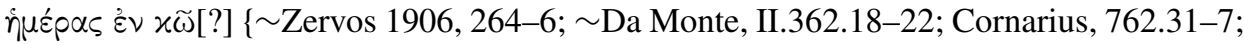
Ｚervos 1906,; Da Monte, II.362.32-364.14; Cornarius, 762.51-764.7; Zervos 1906, 267.14-270.18; Da Monte, II.366.18-23; Cornarius, 766.1-8; Zervos 1906, 270.19 286.3; Da Monte, II.374-376; Cornarius, 772.61-775.42; Zervos 1906, 286.4-14; $\sim$ Da Monte, II.377.11-25; Cornarius, 775.56-776.12; Zervos 1906, 286.15-289.10; $\sim$ Da Monte, II.379.3-384.19; Cornarius, 777.33-782.7; Zervos 1906, 289.11-291.12; $\sim$ Da Monte, II.385.1-19; Cornarius, 782.51-783.13; Zervos 1906, 291.13-292; Da Monte, II.386-438; Cornarius, 784.45-832.8\}. [208v-210r] Table of Contents: Пív $\alpha \xi$

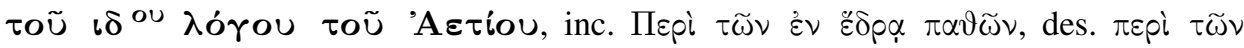

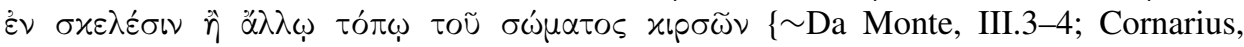

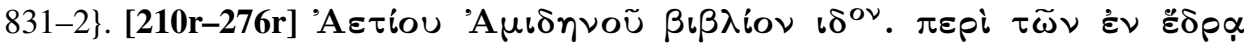

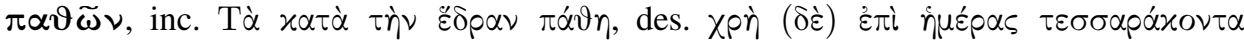
$\dot{\eta} \lambda l \alpha ́ \zeta \varepsilon \sigma \vartheta \alpha_{l} \chi(\alpha \iota) \tau \rho i \beta \varepsilon \sigma \vartheta \alpha l\{$ Da Monte, III.5-69.30; Cornarius, 833-904\}. [276r-277r]

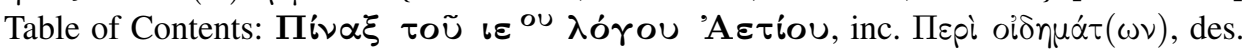

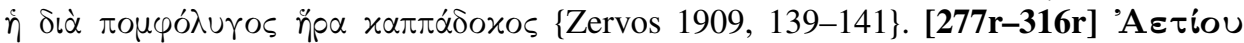

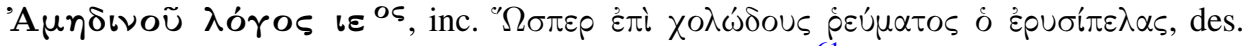

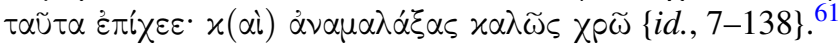

Blank pages: $316 \mathrm{v}-317 \mathrm{v}$.

Annotations (scribal): (32) text erased and corrected - (65) variant reading: ' $\Gamma \rho(\alpha \dot{\varphi} \varepsilon \tau \alpha \mathrm{l})$

61 Zervos, 1911, op. cit. (note 54); Da Monte, op. cit. (note 55); Cornarius, op. cit., (note 55); Daremberg, op. cit. (note 33); Costomiris, op. cit. (note 57); Zervos, 1906, op. cit. (note 58); Zervos, 1909, op. cit. (note 60). 


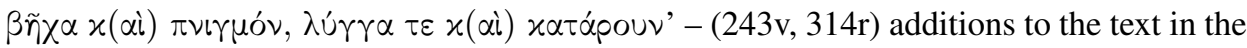
outer margin.

Handwriting: Humanist hand similar to those in Oxford, Corpus Christi College, MSS 97-99. ${ }^{62}$

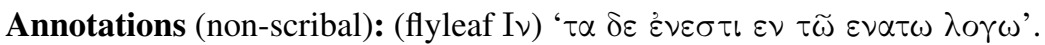

Paper: folded in $2^{\circ}$; chain distance $31 \mathrm{~mm}$; watermarks very similar to Piccard 119080 , 119149, 119155 (anchor), attested in 1509-1511 and to Piccard 123855-8 (crossbow), attested in 1549-55.

Catchwords (scribal): Ii3, vertical.

Quire signatures (non-scribal): Latin letters Ie1 'pp' (1r) through 'zz' (p. 144), then 'aaa' (f. 82r) through 'zzz' (f. 300r), then 'Aaaa' (f. 308r).

Quires: 1 (i), 2 (iii), 1 (iv), $4 \times 8$ (p. 63), $27 \times 10$ (f. 251), 8 (f. 259), $4 \times 10$ (f. 299), 8 (f. 307), 10 (f. 317).

Binding: corio russico. - Flyleaves: (i-iii) chain distance $26 \mathrm{~mm}$, no watermarks. - (iv and 317) same paper as in the MS.

Marks of ownership: (flyleaf Ir) 'Ex Bibliotheca Askeviana / P ii Art 546 / J. Sims'.

Provenance: Anthony Askew (1722-74), London; [his sale, G. Leigh and J. Sotheby, London, 15 March 1785, lot 546]; purchased by James Sims (1741-1820), London, in 1785; purchased by the London Medical Society in 1802; purchased by the Wellcome Library in 1984.

Bibliography: Bibl. Askev., 34 (no. 546); Cat. Med. Soc. 1803, 4; Cat. Med. Soc. 1829, 5; Daremberg, 163; Dawson, 112; Nias, liv; Diels, II.6; Nutton, 24; Nutton and Zipser, 261; Touwaide, 539

Georgios A. Costomiris, 'Études sur les écrits inédits des anciens médecins grecs. Deuxième série: L'Anonyme de Daremberg, Métrodora, Aétius', Revue des Études Grecques, 3 (1890), 145-79: 172.

\section{MS.MSL.112 (olim NN a 11 / Wf 8)}

London, ca. 1732-63 AD (from watermarks).

Paper, $320 \times 195, \mathrm{v}+217+$ vii (foliated 1-217), linn. 30 [244 $\times 141]$

John Zacharias Aktouarios, Medical Epitome ${ }^{63}$ 3, first part of 6, 4, 5.

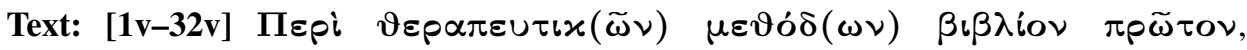

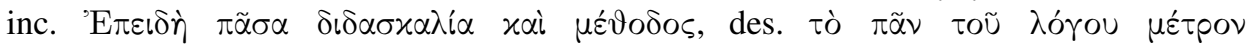

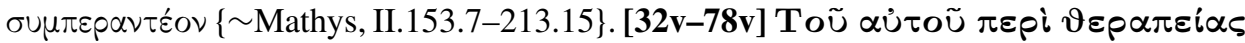

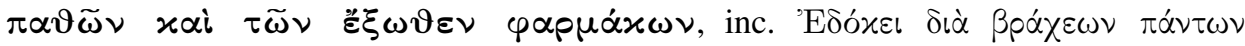

62 Cf. Nigel G. Wilson, A Descriptive Catalogue of the Greek Manuscripts of Corpus Christi College Oxford (Cambridge: D.S. Brewer, 2011), 16-17 with pls. 33, 35-36. This reference was kindly pointed out to me by Rudolf Stefec.

63 Books 3-6 have no Greek edition and have been published only in Latin translation: Mathys, op. cit. (note 34). 


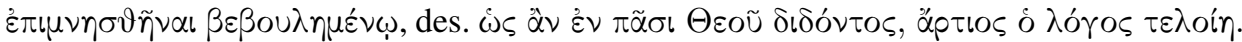

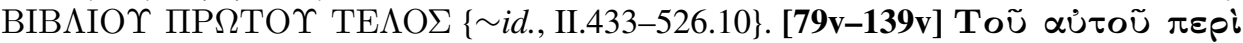

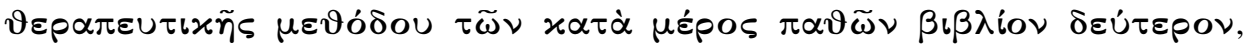

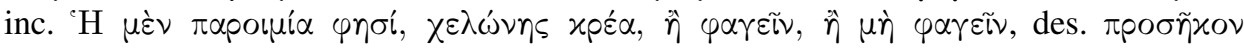

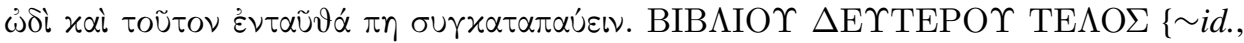

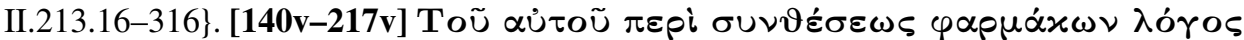

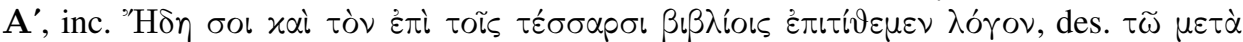

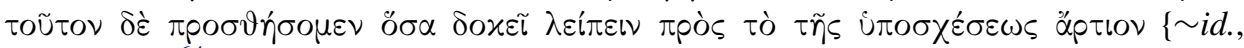
II. $317-432\} .^{64}$

Note: This MS was copied directly from Wellcome MS.MSL.52 (203r-332v). Several labels bound among the pages of MS.MSL.112 contain handwritten notes in which the scribe corrects errors he had made while copying from MS.MSL.52 and refers directly to his exemplar. For example, the label for f. 166v in MS.MSL.112 reads ' $\beta$ เ $\beta \lambda$ iou -

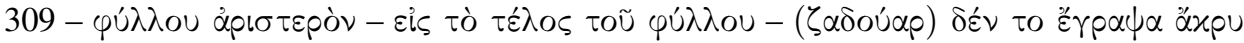

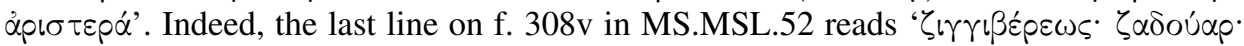

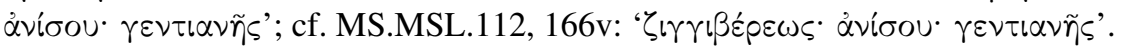

Paper: folded in $2^{\circ}$; chaindistance $25 \mathrm{~mm}$; PRO PATRIA watermarks with GR countermarks similar to Gaudriault 314.1-2. ${ }^{65}$

Marks of ownership: (flyleaf II $r$ ) 'Ex Bibliotheca Askeviana / Part ii. Art. 541 / J. Sims'.

Provenance: Anthony Askew (1722-74), London; [his sale, G. Leigh and J. Sotheby, London, 15 March 1785, lot 541]; purchased by James Sims (1741-1820), London, in 1785; purchased by the London Medical Society in 1802; purchased by the Wellcome Library in 1984.

Bibliography: Bibl. Askev., 33 (no. 541); Cat. Med. Soc. 1803, 3; Cat. Med. Soc. 1829, 4; Dawson, 114; Nias, liv; Diels, II.110; Nutton, 24; Nutton and Zipser, 261; Touwaide, 538; Bouras-Vallianatos, op. cit. (MS.MSL.52) 370-1, 400.

\section{MS.MSL.114 (olim AA c 2 / Wf 15)}

Byzantine Empire, ca. 1335-45 AD (from watermarks).

Paper, $295 \times 230$, iii + 199 + iii (foliated 1-128, 129-201), linn. 36 [225 × 160], unclear ruling.

Paul of Aegina, Medical Epitome (TLG 0715.001).

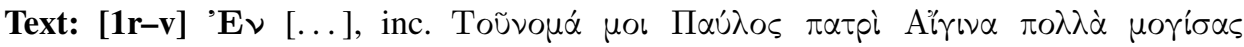

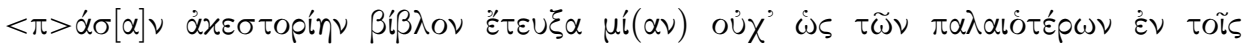

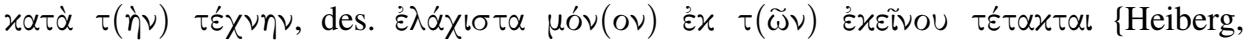

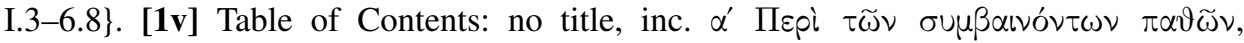

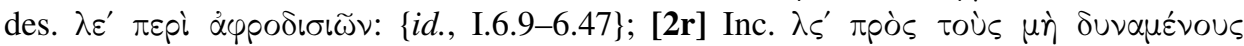

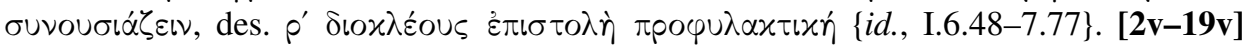

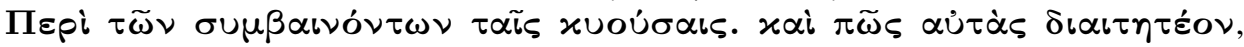

${ }^{64}$ Mathys, op. cit. (note 34).

65 Raymond Gaudriault, Filigranes et autres caractéristiques des papiers fabriqués en France aux XVIIe et XVIIIe siècles (Paris: CNRS; J. Telford, 1995). 


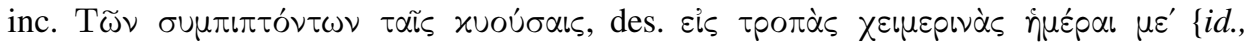

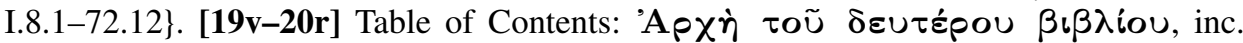

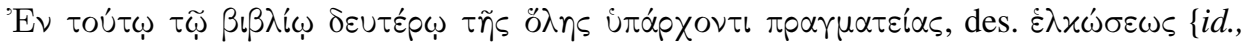

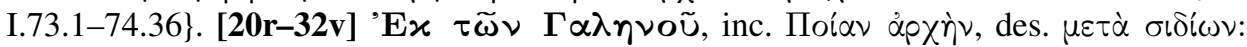

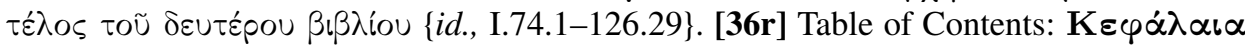

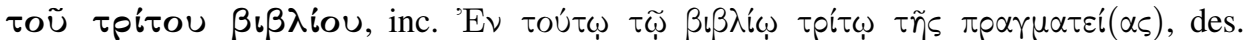

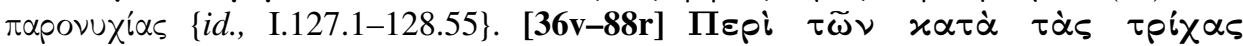

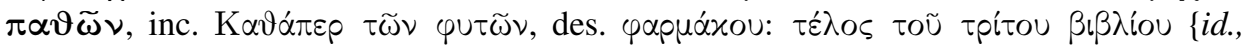

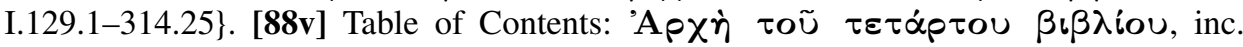

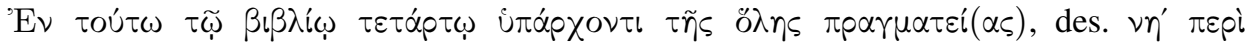

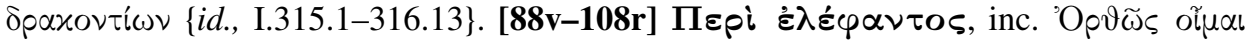

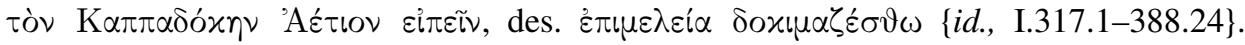

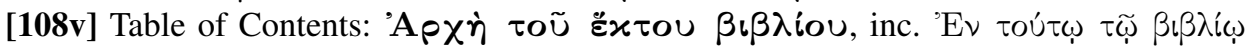

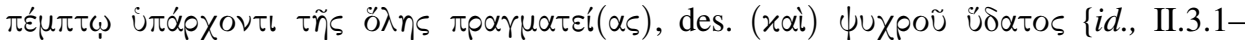

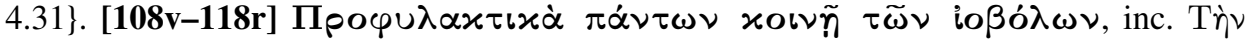

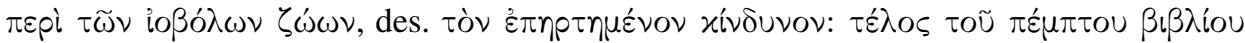

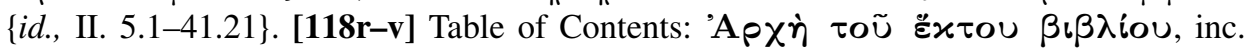

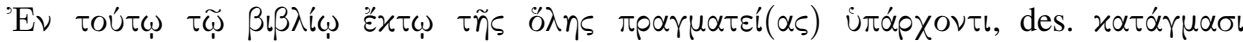

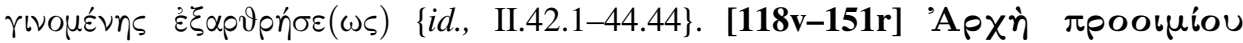

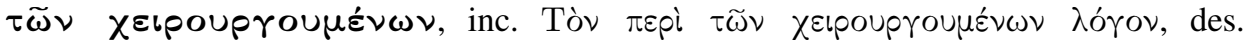

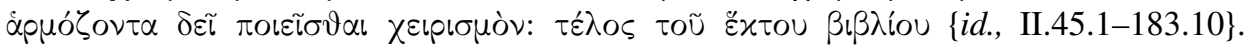

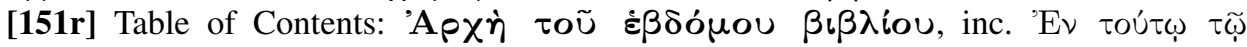

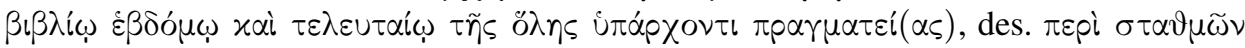

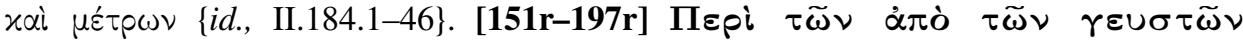

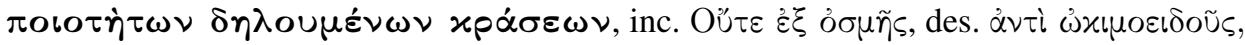

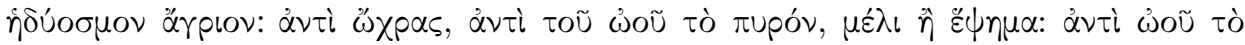

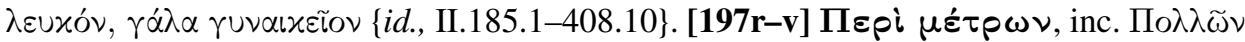

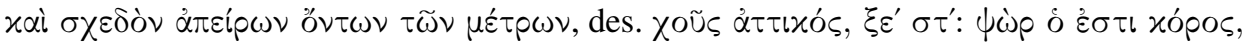
$\mu \delta^{\prime} \lambda^{\prime}\left\{\sim i d .\right.$, II.408.11-411.20\}. ${ }^{66}$

Annotations (scribal): (36r, 68r, 77r, 97r, 122r, 140v, 146r, 184v, 187r) additions to the

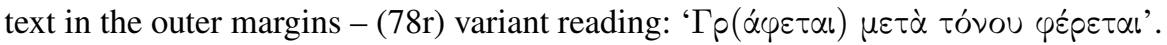

Handwriting: attributed by Nigel Wilson to Georgios Chrysokokkes (RGK III 126).

Text added by several different hands: [1r-v] Lost text replaced. [2r, lower margin]

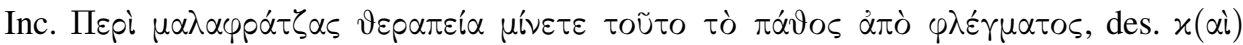

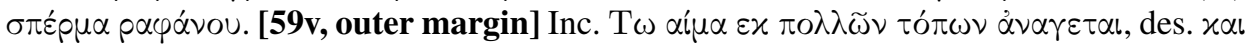

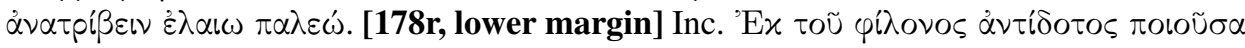

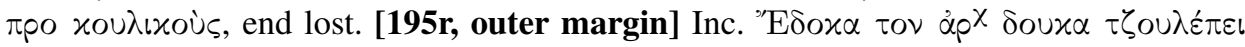

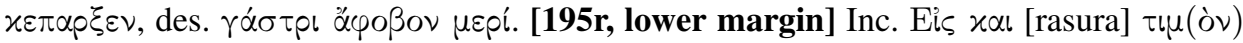

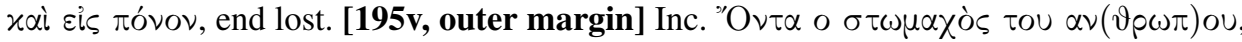

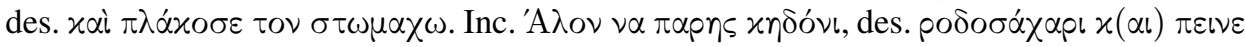

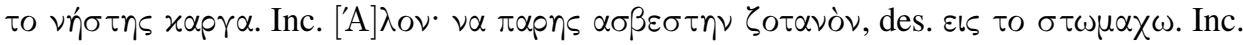

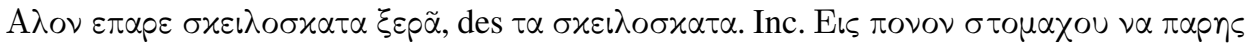

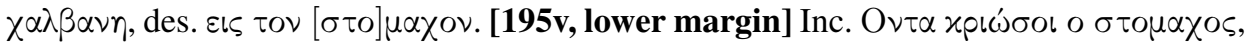

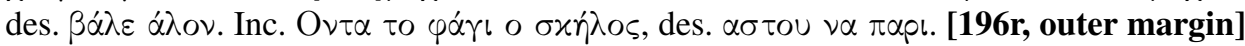

${ }^{66}$ Heiberg, op. cit. (note 35). 


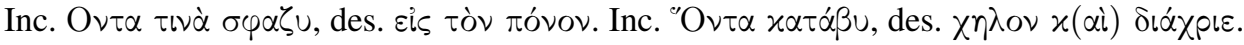

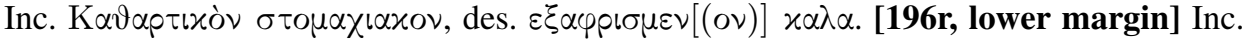

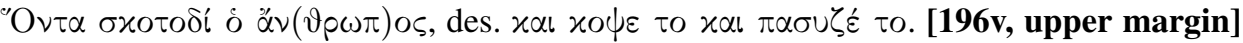

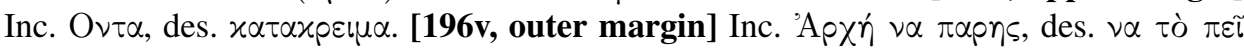

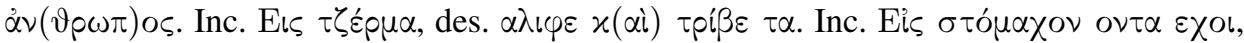

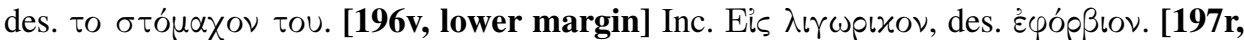

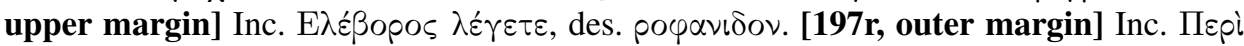

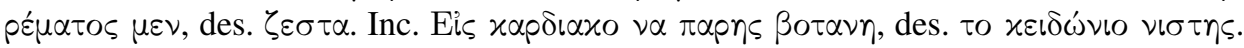

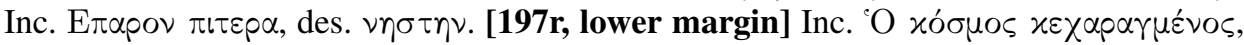

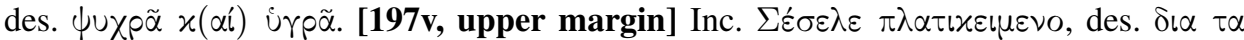

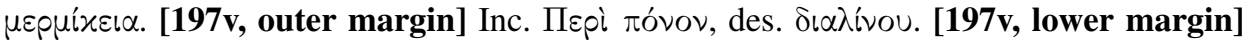

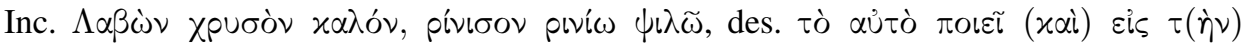

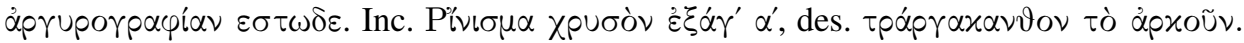

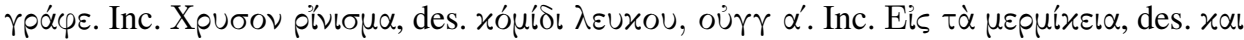

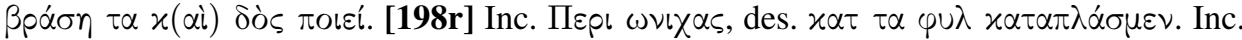

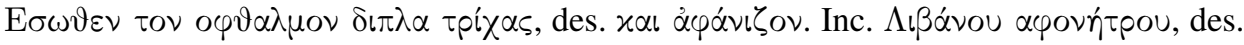

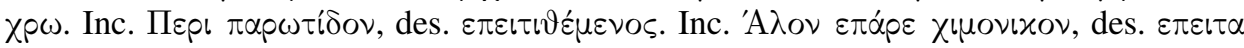

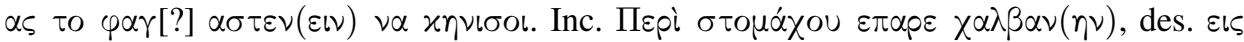

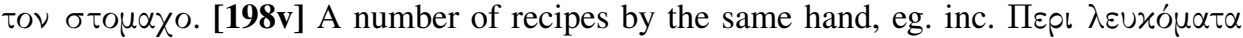

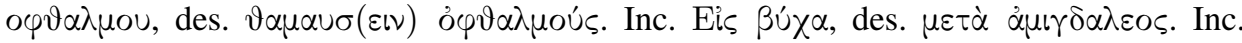

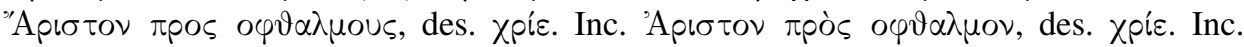

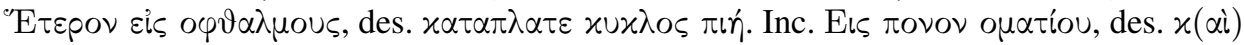

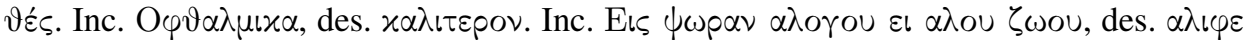

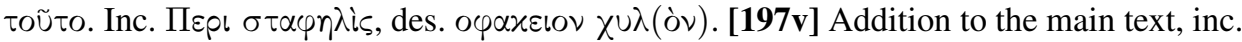

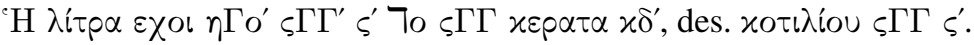

Annotations (non-scribal): (16r, 18v, 20r, 20v, 22v, 26r, 29v, 32r, 36r, 37v, 39v, 40r, $43 \mathrm{v}, 44 \mathrm{v}, 48 \mathrm{r}, 48 \mathrm{v}, 49 \mathrm{r}, 49 \mathrm{v}, 50 \mathrm{r}, 51 \mathrm{r}, 52 \mathrm{r}, 52 \mathrm{v}, 53 \mathrm{r}, 53 \mathrm{v}, 54 \mathrm{v}, 55 \mathrm{r}, 55 \mathrm{v}, 56 \mathrm{r}, 57 \mathrm{r}, 58 \mathrm{r}$, $58 \mathrm{v}, 62 \mathrm{r}, 62 \mathrm{v}, 63 \mathrm{v}, 65 \mathrm{r}, 67 \mathrm{v}, 68 \mathrm{r}, 68 \mathrm{v}, 69 \mathrm{v}, 73 \mathrm{r}, 74 \mathrm{r}, 75 \mathrm{v}, 76 \mathrm{r}, 76 \mathrm{v}, 91 \mathrm{r}, 92 \mathrm{r}, 93 \mathrm{r}, 94 \mathrm{r}$, $94 \mathrm{v}, 97 \mathrm{v}, 100 \mathrm{r}, 101 \mathrm{r}, 101 \mathrm{v}, 102 \mathrm{v}, 104 \mathrm{r}, 106 \mathrm{v}, 109 \mathrm{v}, 151 \mathrm{v}, 152 \mathrm{r}, 152 \mathrm{v}, 153 \mathrm{r}, 153 \mathrm{v}, 154 \mathrm{r}$, $154 \mathrm{v}, 155 \mathrm{v}, 156 \mathrm{r}, 156 \mathrm{v}, 157 \mathrm{r}, 157 \mathrm{v}, 158 \mathrm{r}, 158 \mathrm{v}, 159 \mathrm{r}, 159 \mathrm{v}, 160 \mathrm{r}, 160 \mathrm{v}, 161 \mathrm{r}, 161 \mathrm{v}, 162 \mathrm{r}$, $162 \mathrm{v}, 163 \mathrm{r}, 163 \mathrm{v}, 165 \mathrm{r}, 165 \mathrm{v}, 166 \mathrm{r}, 166 \mathrm{v}, 167 \mathrm{r}, 167 \mathrm{v}, 168 \mathrm{r}, 168 \mathrm{v}, 169 \mathrm{r}, 169 \mathrm{v}, 170 \mathrm{r}, 170 \mathrm{v}$, $171 \mathrm{r}, 171 \mathrm{v}, 172 \mathrm{r}, 172 \mathrm{v}, 173 \mathrm{r}, 173 \mathrm{v}, 174 \mathrm{r}, 174 \mathrm{v}, 175 \mathrm{r}, 176 \mathrm{r}, 177 \mathrm{r}, 177 \mathrm{v}, 178 \mathrm{v}, 179 \mathrm{r}, 179 \mathrm{v}$, 180r, 180v, 181r, 182r, 183r, 183v, 185r, 185v, 186r, 189v, 190v, 193r, 193v) mostly short phrases on single words, of four kinds: efficacy phrases, indication of contents or, less frequently, short explanatory phrases and variant readings, eg. (18v) ' $\alpha \dot{\rho} \iota \tau \tau \omega \nu$ ह่ $\pi \varepsilon เ \sigma \tau \omega \lambda \eta \dot{\eta} \nu$

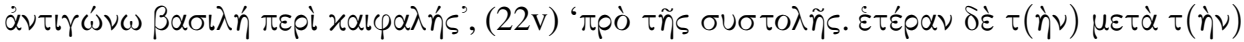

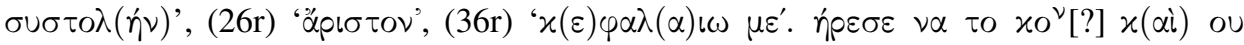

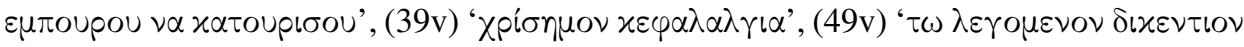

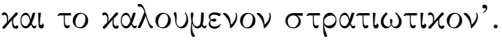

Paper: folded in $2^{\circ}$; chain distance $43 \mathrm{~mm}$; watermarks (1) very similar to Mošin and Traljić 6947, 6949, 6956 (saucisson), attested in 1338-50 and (2) identical with Mošin and Traljić 5791 (licorne), attested in 1339-44. ${ }^{67}$

Added leaves: (ff. 1, 198) paper folded in $2^{\circ}$; chain distance $35 \mathrm{~mm}$; watermarks similar to Piccard 122415 (scissors), attested in 1457.

${ }^{67}$ Mošin and Traljić, op. cit. (note 24). 
Quires: 1 (flyleaf i), 2 (ii-iii), 1 (f. 1), 4 (f. 5), $2 \times 1$ (f. 7), $23 \times 8$ (f. 190), 1 (f. 191), 6 (f. 197), 1 (f. 198), 2 (i-ii), 1 (iii).

Binding: corio russico. - Flyleaves: chain distance $25 \mathrm{~mm}$, no watermarks.

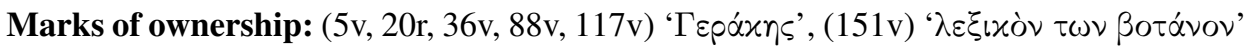
- (flyleaf II $r$ ) 'Ex Bibliotheca Askeviana P.ii.Art. 404 / J. Sims / A A Ac c 2 / WE15' (flyleaf III $r$ ) 'W. H. 18' - (back pastedown) label inscribed 'O.g.24'.

Provenance: (Manuel Kantakouzenos?) Gerakes (cf. Wellcome MS.MSL.52) - Anthony Askew (1722-74), London; [his sale, G. Leigh and J. Sotheby, London, 10 March 1785, lot 404]; purchased by James Sims (1741-1820), London, in 1785; purchased by the London Medical Society in 1802; purchased by the Wellcome Library in 1984.

Bibliography: Bibl. Askev., 23 (no. 404); Cat. Med. Soc. 1803, 3; Cat. Med. Soc. 1829, 4; Daremberg, 164; Nias, lvi-lvii; Diels, II.77; Dawson, 115-6; Nutton, 24-5; Nutton and Zipser, 261; Touwaide, 538-9.

Brigitte Mondrain, 'La lecture et la copie de textes scientifiques à Byzance pendant l'époque paléologue', in Giuseppe De Gregorio and Maria Galante (eds), La produzione scritta tecnica e scientifica nel Medioevo: libro e documento tra scuole e professioni (Spoleto: Centro italiano di studi sull'alto medioevo, 2012), 607-32: 632.

Gabrielle Lherminier, 'Edition critique et traduction annotée du livre V des "Epitomae Medicae" de Paul d'Egine' (unpublished PhD thesis: Paris, École Pratique des Hautes Études, 2013), 249-50.

\section{MS.MSL.124 (olim AA d 6 / Wf 16)}

England, c. $1650 \mathrm{AD}$ (from watermarks).

Paper, $310 \times 208, \mathrm{i}+77+\mathrm{i}$ (foliated 1-77), linn. 45 [240 × 137].

John Zacharias Aktouarios, On Urines (TLG 3188.002).

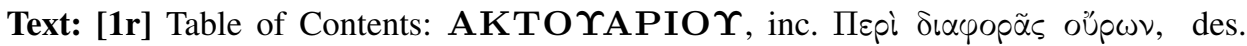

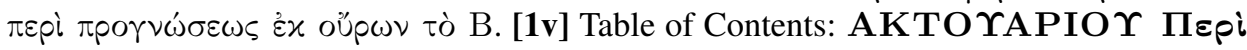

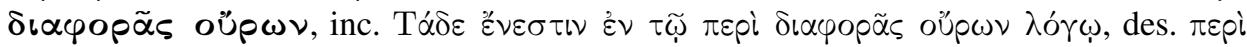

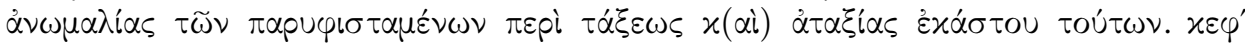

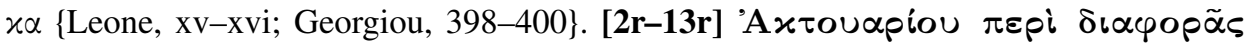

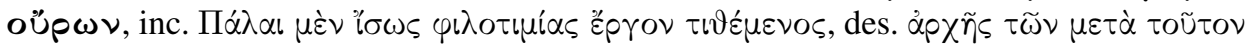

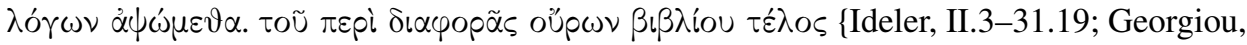
401-56\}. [13v] AKTO

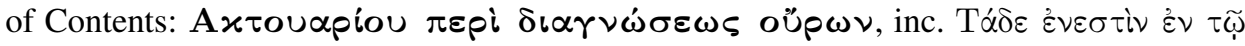

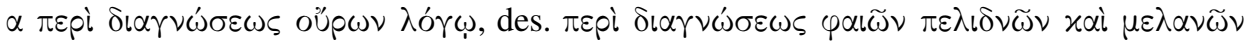

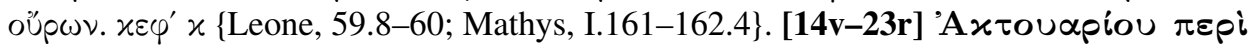

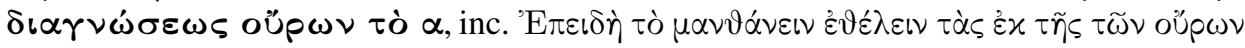

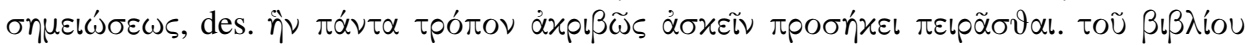

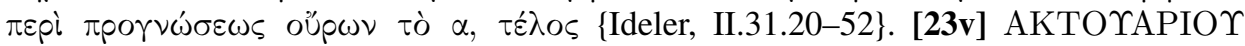

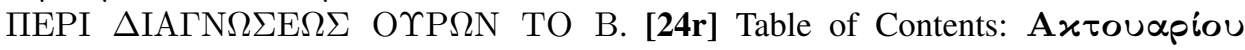

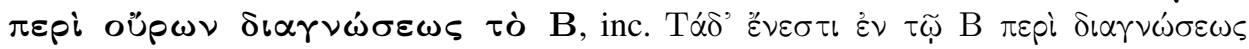




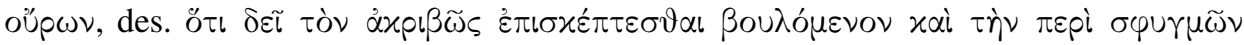

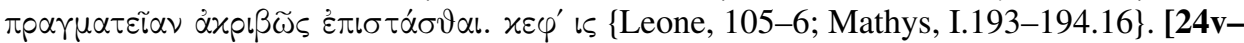

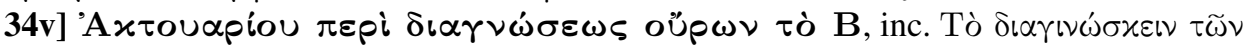

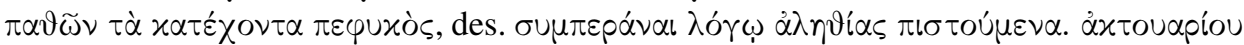

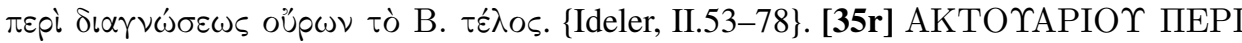

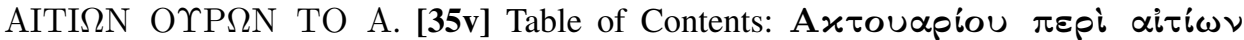

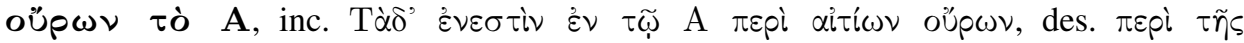

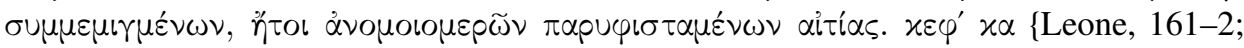

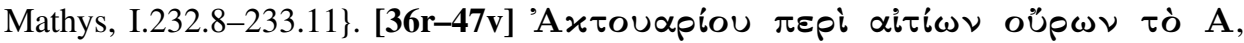

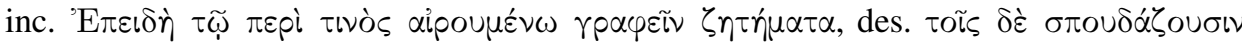

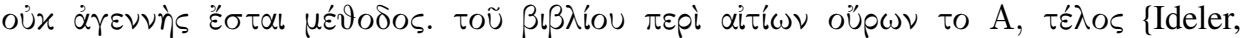

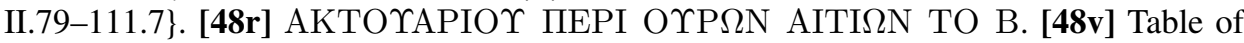

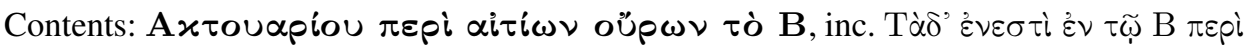

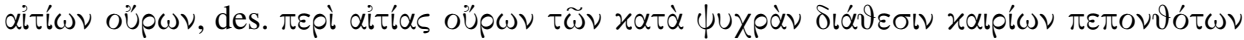

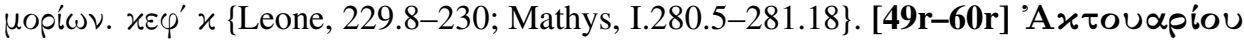

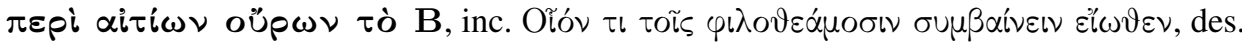

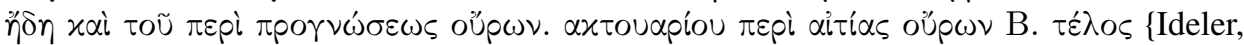

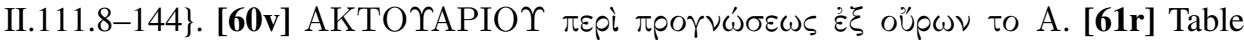

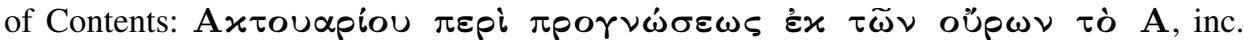

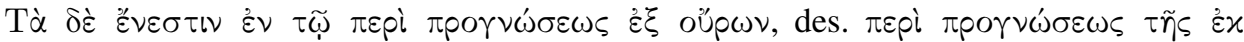

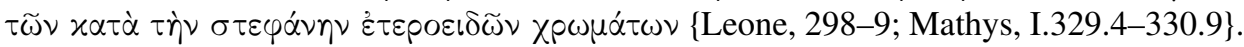

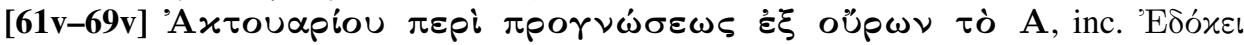

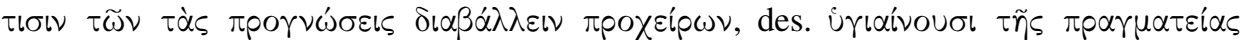

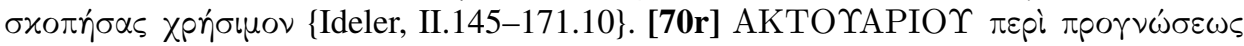

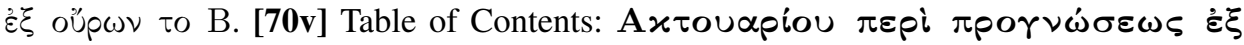

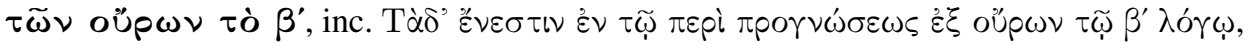

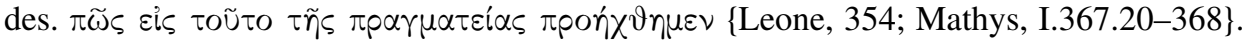

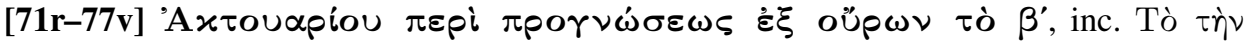

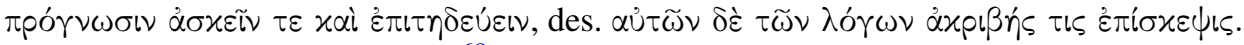
$\mathrm{TE} \Lambda \mathrm{O} \Sigma$ \{Ideler, II.171.11-192\}. ${ }^{68}$

Note: Stavroula Georgiou has demonstrated that this MS was copied directly from Cambridge, Gonville and Caius College, MS 76/43.

Paper: folded in $2^{\circ}$; chain distance $27 \mathrm{~mm}$; watermark very similar to Nostitz 760 (three hats with countermark NZ), attested in $1650 .{ }^{69}$

Provenance: Anthony Askew (1722-74), London; [his sale, G. Leigh and J. Sotheby, London, 15 March 1785, lot 542 (?)]; probably purchased by James Sims (1741-1820), London, in 1785; probably purchased by the London Medical Society in 1802; purchased by the Wellcome Library in 1984.

Bibliography: Bibl. Askev., 33 (no. 542); Cat. Med. Soc. 1803, 3; Cat. Med. Soc. 1829, 3; Daremberg, 158; Costomiris 10, 441; Nias, liv; Diels, II.109; Dawson, 120-1;

68 Leone, op. cit. (note 34); Georgiou, op. cit. (note 34); Ideler, op. cit. (note 23); Mathys, op. cit. (note 34).

69 The Nostitz Papers: Notes on Watermarks Found in the German Imperial Archives of the 17th \& 18th Centuries, and Essays Showing the Evolution of a Number of Watermarks (Hilversum: Paper Publications Society, 1958). 
Nutton, 24; Nutton and Zipser, 261; Touwaide, 539; Georgiou, op. cit. (note 34), 254-5, 332-4.

John Symons (ed.), Books from the Library of the Medical Society of London: An Exhibition, 14 January to 3 April (London: Wellcome Institute, 1985), 9.

\section{MS.MSL.126 (olim AA c 4 / Wf 6)}

Cambridge, 1648 AD (from note on f. 1r).

Paper, $320 \times 212$, i $+482+\mathrm{i}$ (foliated 1-161, 162-245, 245-302, 303-307, 308-314, 315-386, 387-409, 410, 411-474).

Oribasios, Medical Collections (TLG 0722.001), Books 1-10 and 14.

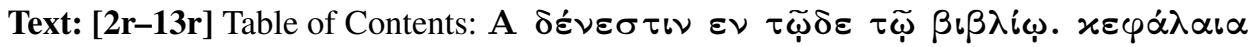

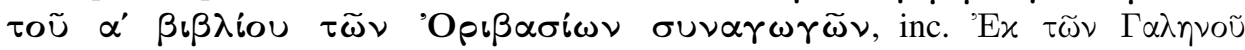

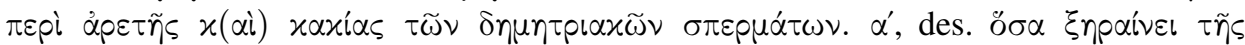

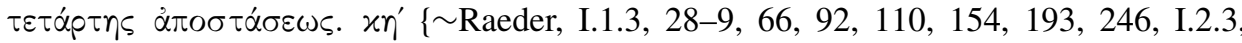

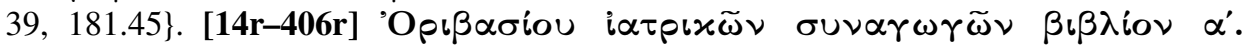

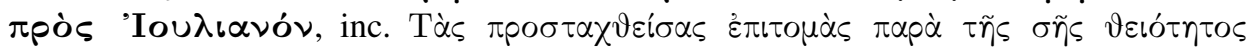

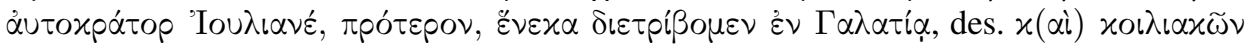

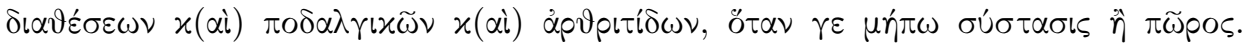

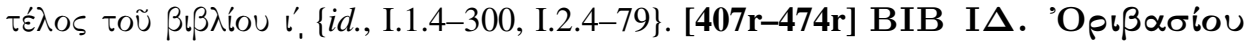

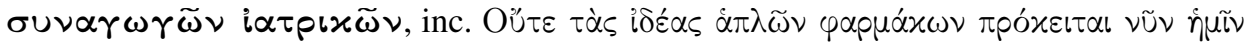

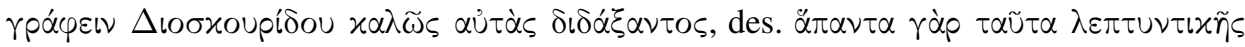

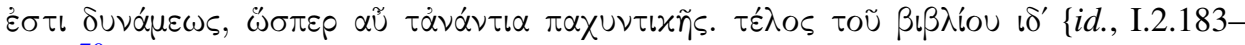
$237\}$. $^{70}$

Note: This manuscript was copied directly from Cambidge, Saint John's College, MS A.6.

Marks of ownership: (1r) 'Vita Ovidii Oribasii describitur a Eunapium graecae, qui

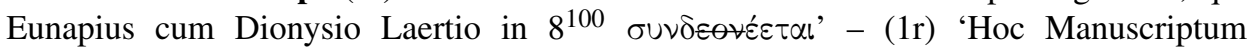
Transcribebatur ex Copiae Bibliothecae St Johanntis Collegii: Acad. Cantab. Atque Reuisum fuit secundum Copiam Anno Domini 1648. Robertus Waideson, Medicinae Doctor' - (1r) 'Ex Bibliotheca Askeviana / P. ii. Art. 588 / J. Sims'.

Provenance: probably commissioned by Robert Wadeson, Cambridge - Anthony Askew (1722-74), London; [his sale, G. Leigh and J. Sotheby, London, 16 March 1785, lot 588]; purchased by James Sims (1741-1820), London, in 1785; purchased by the London Medical Society in 1802; purchased by the Wellcome Library in 1984.

Bibliography: Bibl. Askev., 37 (no. 588); Cat. Med. Soc. 1803, 149; Cat. Med. Soc. 1829, 232; Daremberg, 158; Nias, lv-lvi; Diels, II.71; Raeder, op. cit. (note 70), Vol. I.1, v; Dawson, 122-3; Nutton, 24; Nutton and Zipser, 261; Touwaide, 539.

70 Johannes Raeder, Oribasii Collectionum medicarum reliquiae, 2 vols [CMG, VI/1,1-2,2] (Leipzig: Teubner, 1928-33). 


\section{MS.MSL.135 (olim H Hi 1)}

Ottoman Empire, ca. 1525-65 AD (from watermarks and handwriting style).

Paper, $210 \times 150, \mathrm{i}+162+\mathrm{i}($ foliated $1-162),{ }^{71}$ linn. 22 [156 × 90], $20 \mathrm{D} 1$.

[1r-86r] Theophanes Chrysobalantes (Nonnos), Medical Epitome. ${ }^{72}$ [86r-96r] Idem, On Diet (TLG 0721.010, 0721.017). ${ }^{73}$ [96r-110r] Idem, Synopsis on Composite Drugs. ${ }^{74}$ [110v-154v] Symeon Seth, On the Capacities of Foodstuffs (TLG 3113.002). ${ }^{75}$

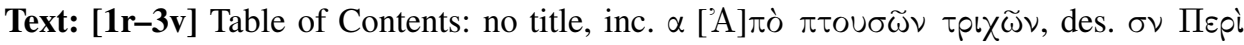

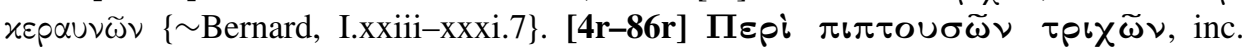

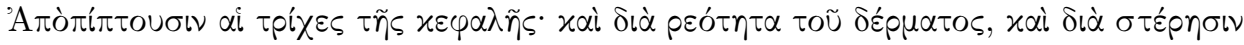

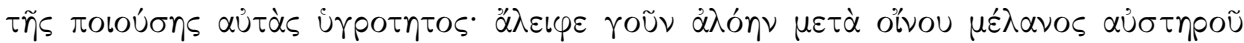

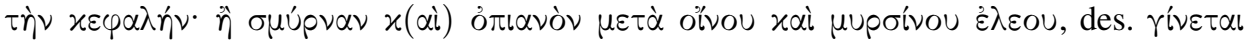

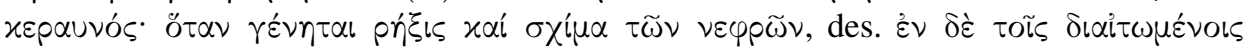

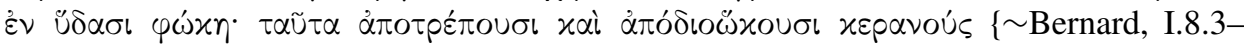

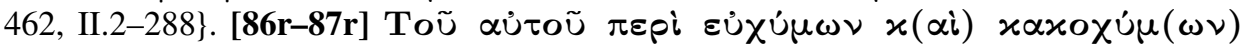

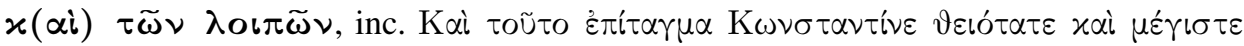

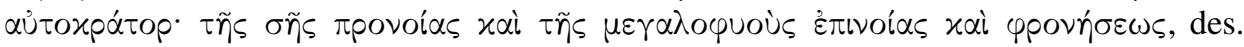

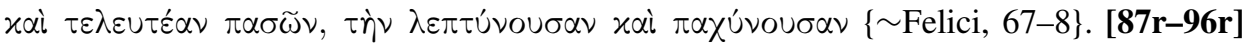

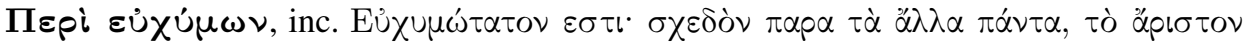

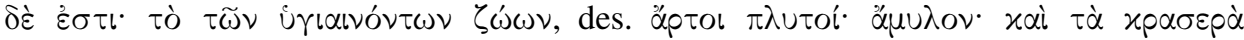

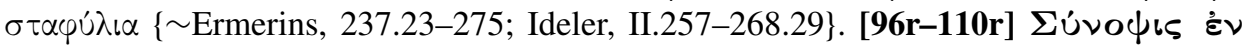

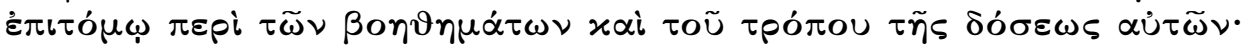

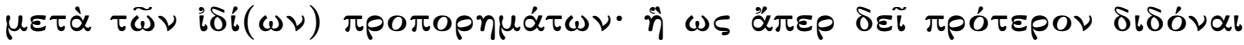

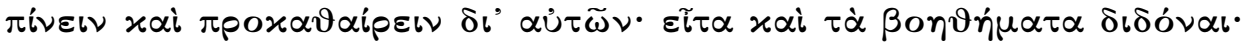

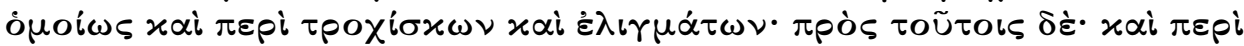

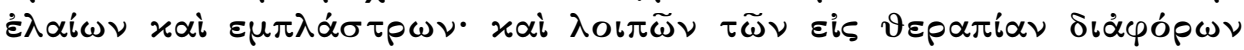

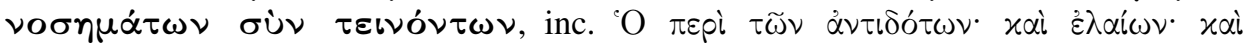

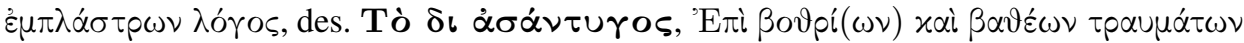

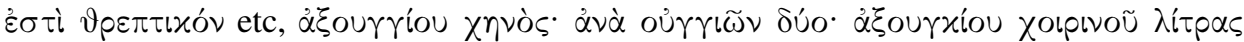

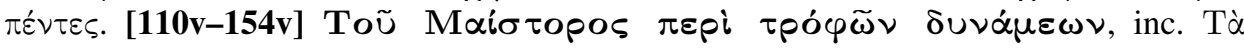

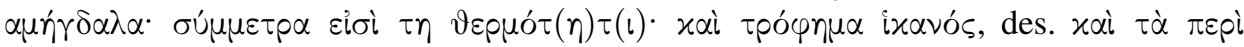

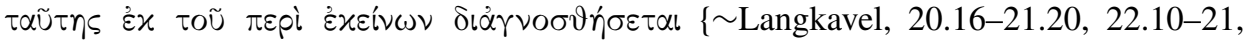
20.7-15, 23.9-21, 24.3-25.2, 23.23-24.2, 26.1-28.6, 29.23-8, 30.11-31.6, 34.1-39.23,

\footnotetext{
71 The current folia 1-16 have an earlier foliation, 1-12.

72 The order of chapters is sometimes the same as that in the edition by Johann Stefan Bernard, Theophanis Nonni Epitome de curatione morborum, 2 vols (Gotha: Ettinger, 1794-5). However, there are also lots of variations on the text (which is usually longer) and the headings; the proem and last chapters on venomous animals are not included.

73 The text is close to Ideler's edition, op. cit. (note 34), Vol. 2, 257-81, although shorter, and its end coincides with Ermerins' edition, op. cit. (note 23), 225-75; the proem preceding the text is similar to the one edited by Laura Felici, 'L'opera medica di Teofane Nonno in manoscritti inediti', Acta medicae historiae Patavina, 28 (1981-2), 59-74: 67-8.

74 The introduction follows the edition of the relevant part printed by Felici, op. cit. (note 73), 63, based on Marcianus gr. V 16, ff. 129r-v, 140-146, and Vaticanus gr. 292, ff. 68v-96. First recipe on theriac; last recipe on the plaster called $\mu \alpha \sigma \tau \iota \chi \eta n \alpha \dot{~}$

75 The order of chapters is largely different from Langkavel's edition, op. cit. (note 48); chapters usually appear

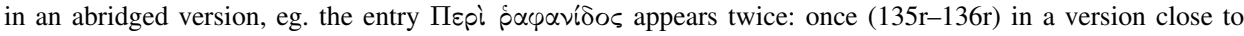
Langkavel's edition and one more (114r) in an abridged form.
} 
40.9-23, 42.23-43.11, 90.11-92.13, 43.19-45.12, 48.4-55.4, 57.14-25, 67.9-12, 58.1-16, 56.19-57.18, 58.19-59.14, 60.6-13, 61.8-62.24, 63.9-67.19, 71.14-6, 68.8-71.6, 71.1773.3, 73.12-74.3, 73.4-11, 74.4-80.3, 81.5-11, 86.21-87.4, 80.24-81.4, 80.4-23, 84.3$85.22,87.5-88.12,85.23-86.20,83.15-84.2,81.12-83.14,88.13-93.25,100.5-12,94.1-$ $100.4,100.13-103.7,103.25-104.3,103.8-103.24,104.12-107.10,108.1-22,107.11-24$, 108.23-109.10, 111.10-26, 109.11-111.9, 112.1-123.15, 18.4-20.6, 123.16-125\}. [154v]

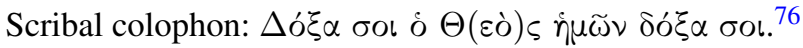

Note: According to Barbara Zipser, MS.MSL.135 is a sibling of Florence, Biblioteca Medicea Laurenziana, MS Plut. 75.6.

Blank pages: $156 \mathrm{r}-161 \mathrm{v}, 162 \mathrm{v}$.

Handwriting: unidentified post-Byzantine hand.

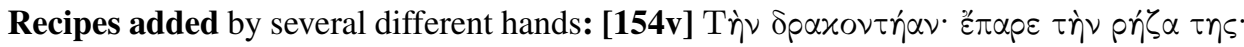

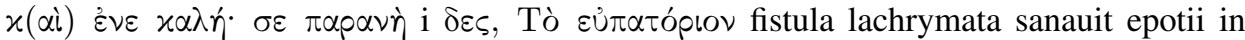

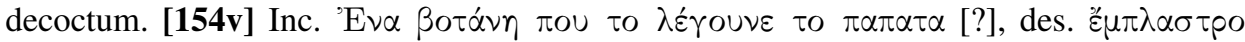

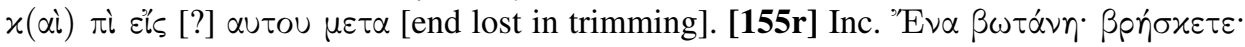

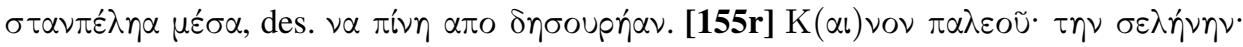

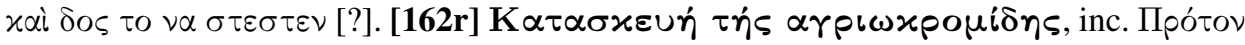

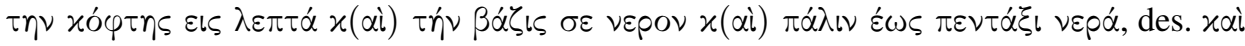

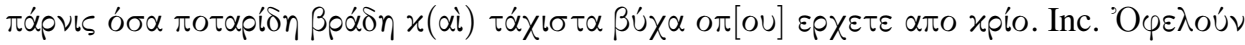

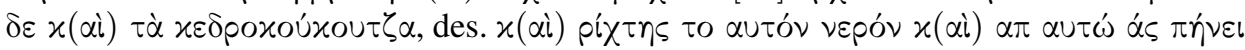

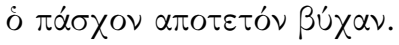

Annotations (non-scribal): marginal notes by various hands, usually in the vernacular and mainly of three kinds: short explanations in the form of synonyms, indications of

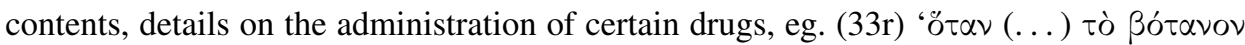

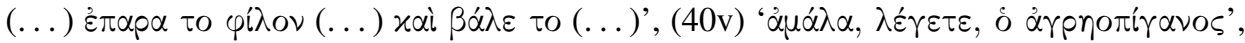

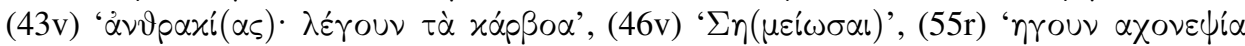

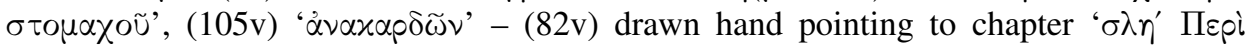

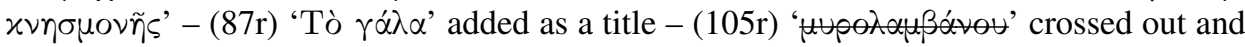

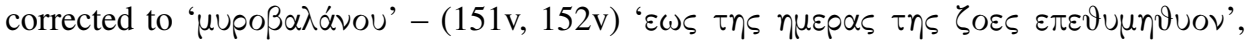

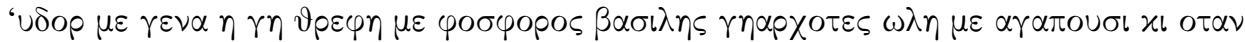

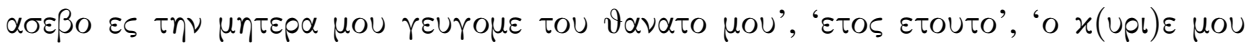
$\chi(\alpha l) \Theta \varepsilon \varepsilon \mu o u$ ' - (155v) 'Amico mio paga volto ecca[?] choritio[?]. Ama i dio non fallire far pur bene e lasca dire'. ${ }^{77}$

Paper: folded in $4^{\circ}$, chain distance $32 \mathrm{~mm}$, watermarks very similar to Mošin 647, 727, 733,748 and 801 , attested respectively in $1495,1528,1530,1535-45$ and $1560-5 .^{78}$

Binding: of blind-tooled brown leather over cardboard; four ridges on the spine. Pastedowns and flyleaves: replaced, chaindistance ca. $26 \mathrm{~mm}$, partly preserved watermark very similar to Heawood 1743 (fleur-de-lis), attested in 1767. - F. 156: chain distance

\footnotetext{
76 Bernard, op. cit. (note 71); Felici, op. cit. (note 72); Ermerins, op. cit. (note 23); Ideler, op. cit. (note 23); Langkavel, op. cit. (note 48).

77 The second sentence is a saying attributed to Saint Crispin of Viterbo (1668-1750).

78 Vladimir Mošin, Anchor Watermarks (Amsterdam: Paper Publication Society, 1973); and Heawood, op. cit. (note 14).
} 
$23 \mathrm{~mm}$, watermark similar to Heawood 302 (circles), attested in 1727-51. - Ff. 157-162: chain distance $28 \mathrm{~mm}$, watermark three crescents.

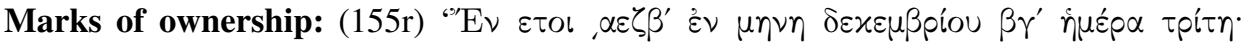

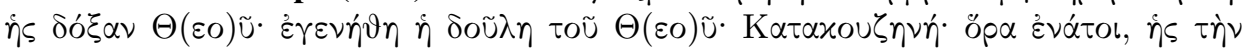

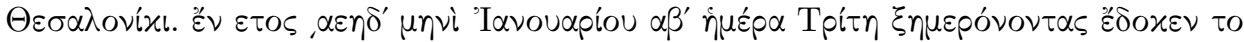

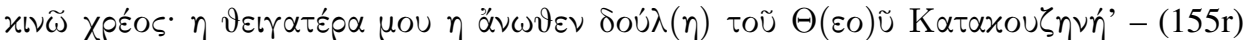

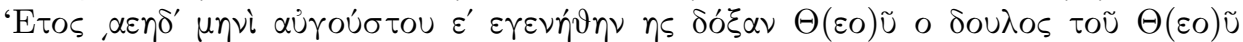

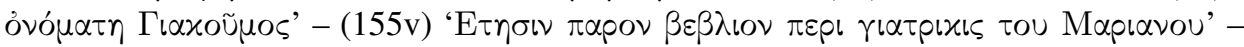
$(155 \mathrm{v})$ 'Andreas Charagas'.

Provenance: London Medical Society (acquired most probably between 1803 and 1829); purchased by the Wellcome Library in 1984.

Bibliography: Cat. Med. Soc. 1829, 201; Nias, lvi; Dawson, 130-1; Touwaide, 539.

\section{MS.289}

Italy, ca. $1535 \mathrm{AD}$ (from watermarks).

Paper, $210 \times 148, \mathrm{i}+30+\mathrm{i}$ (foliated 1-30), linn. 25 [150 × 95], 02D1.

Ps.-Galen, Medical Definitions (TLG 0530.041). ${ }^{79}$

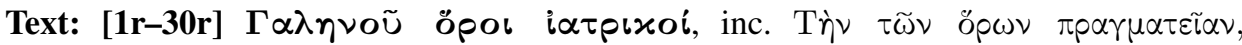

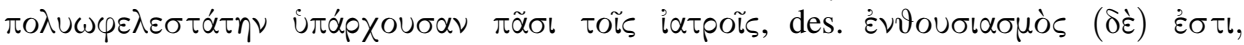

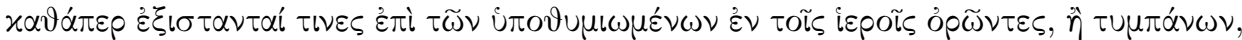

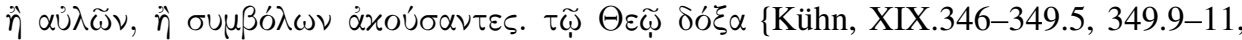
349.18-351.7, 352.5-364.15, 365.8-376.4, 378.4-416.6, 419.12-4, 420.11-421.3, 419.811, 421.4-12, 423.4-6, 423.14-7, 416.7-419.7, 419.15-420.10, 423.7-13, 423.18-424.6, $421.13-423.3,424.7-428.8,351.8-352.4,428.9-462\} .{ }^{80}$

Blank page: $30 \mathrm{v}$.

Annotations (scribal): (10r, 11v, 12v, 16v, 17v, 20r, 26r, 28v, 29v) additions to the main

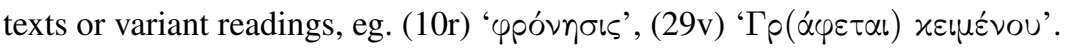

Handwriting: unidentified Renaissance hand.

Paper: folded in $4^{\circ}$; chain distance $35 \mathrm{~mm}$; watermark similar to Sosower balance 2 , attested in $1534 .^{81}$

Quire signatures (scribal): Greek numerals $\operatorname{Im} 3$ ( $\beta^{\prime}$ on f. 8v) and $\operatorname{Im} 1$ ( $\gamma^{\prime}$ on f. 17r, $\delta^{\prime}$ on f. 25r). - IC XC written, in the scribe's hand, in the middle of each page's upper margin.

Quires: $3 \times 8$ (f. 24), $2 \times 1$ (f. 26), 4 (f. 30) [no text lost].

Binding: of cardboard, modern. - Flyleaves and pastedowns: machine-made paper.

Marks of ownership: (1r) 'F. L. 2711' - (1r) '43753A' - (4r) erased and illegible note.

79 The order of paragraphs sometimes does not match Kühn's edition, op. cit. (note 33), Vol. 19, 346-462; some chapters appear in an abridged version.

${ }^{80}$ Kühn, op. cit. (note 33).

81 Mark Sosower, Signa officinarum chartariarum in codicibus Graecis saeculo sexto decimo fabricatis in bibliothecis Hispaniae (Amsterdam: Haakert, 2004). 
Provenance: [R. Lier \& Co, Milan]; purchased on the behalf of Wellcome Library in 1925 (accession number 43753A).

Bibliography: Moorat, I.189; Touwaide, 539.

\section{MS.354}

Paper, $274 \times 210, \mathrm{i}+107+\mathrm{i}$ (foliated $1-108)$.

[1r-18v] Damascius, Commentary on the Aphorisms of Hippocrates (TLG 0728.001). ${ }^{82}$ [18v-21v] [Hippocrates], Prognosticon (TLG 0627.003). ${ }^{83}$ [22r-107v] Stephen, Commentary on the Prognosticon of Hippocrates (TLG 0728.001). ${ }^{84}$

Note: This MS consists of two distinct parts, described below separately as A and $\mathbf{B}$.

A.

Byzantine Empire, ca. 1400-30 AD (from watermarks).

Linn. 40 [220 × 153], D 32D; (scribe B) linn. 41-45, unruled.

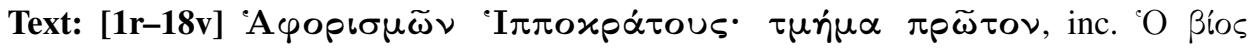

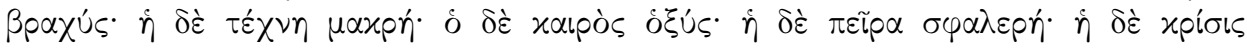

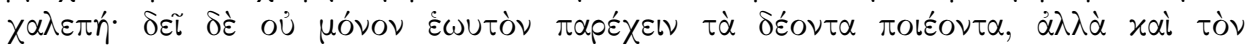

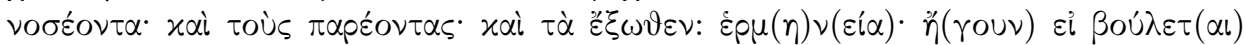

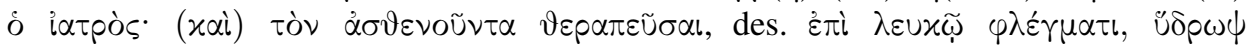

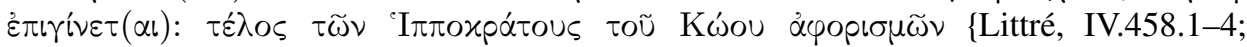
Jones 98.1-5; Dietz, II.250.22-251.21; id., IV.458.5-10; 98.6-13, II.256.8-258.2; id., IV.458.11-460.6; 98.14-100.8, II.260.3-261.8; and so forth up to id., IV.596.8-20; 208.4-7, II.543.14-6; then id., IV.596.9-598.3; 208.8-14; IV.598.5-604.4; 210.1-214.4\}.

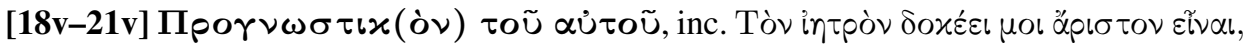

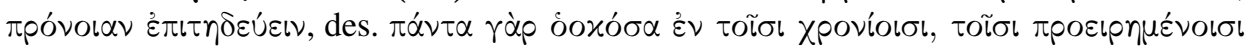

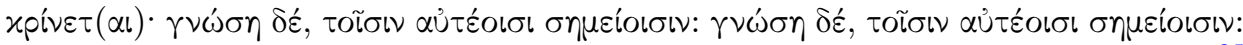

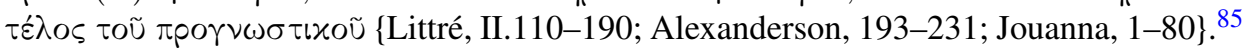

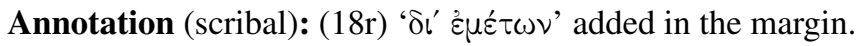

82 The text follows the usual structure of commentaries on Hippocratic Aphorisms providing the aphorism

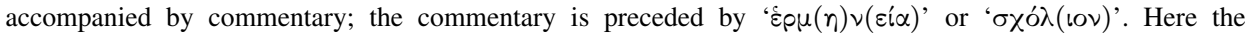
commentary follows the version attributed in other manuscripts to Damascius; Caroline Magdelaine, 'Le commentaire de Damascius aux Aphorismes d'Hippocrate', in Antonio Garzya and Jacques Jouanna (eds), Storia e ecdotica dei testi medici greci: Atti del II Convegno Internazionale, Parigi 24-26 maggio 1994 (Napoli: D'Auria, 1996), 289-306: 292-3. Since the version of the aphorisms in the manuscript is not always identical with the text published by Friedrich Reinhold Dietz, Scholia in Hippocratem et Galenum, 2 vols (Königsberg: Borntraeger, 1834), Vol. 2, 250-543, for each aphorism I provide the references to Littré and Jones, and then to Dietz's edition of Damascius' commentary. The Wellcome MS contains the full text of the commentary published by Dietz, followed (18r-v) by Aphorisms 7.62-3, 65-75 without commentary. Each book has a separate heading (1r, 3v, 6r, 8v, 11r, 14r, 16r).

83 Chapter titles written in the margins of ff. $18 \mathrm{v}-21 \mathrm{v}$.

84 The text in this MS is incomplete and breaks off at the same point (II.1) as in New Haven, Yale University, Cushing/Whitney Medical History Library, MS 50 (olim Saragossa, Cabildo de la Santa Iglesia Mayor del Pilar, MS 1427); John Duffy, Stephani Philosophi In Hippocratis Prognosticum commentariaIII (Berlin: AkademieVerlag, 1983), [CMG, XI/1,2], 18, concludes that the Wellcome MS is a direct copy of the Yale one.

85 Littré, op. cit. (note 38); Jones, op. cit. (note 48); Dietz, op. cit. (note 82); Alexanderson, op. cit. (note 48); Jouanna, op. cit. (note 48). 
Handwriting: two scribes A (1r-18v supra) and B (18v infra-21v).

Paper: folded in $2^{\circ}$; chain distance $35 \mathrm{~mm}$; watermark very similar to Piccard 150435 , 150483, 150564, 150755 (three hills), attested in 1408-27.

Quires: $2 \times 8$ (f. 16), $1+4$ (f. 21) [no text lost].

B.

Venice, Germany or Spain, ca. 1582-7 AD (from watermarks).

Linn. 20 [ca. $212 \times 153$ ], unclear ruling.

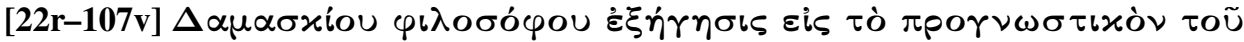

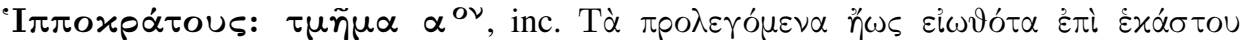

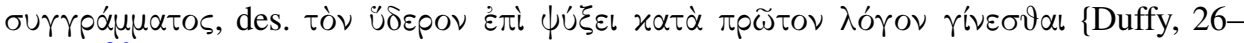
$146.2\} .^{86}$

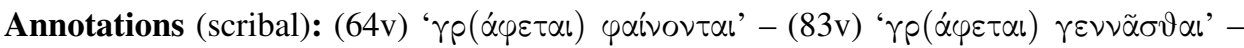

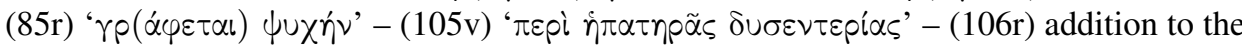

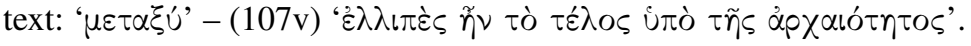

Handwriting: attributed by Otto Kresten to Andreas Darmarios (RGK I 13, II 21, III 22).

Paper: folded in $2^{\circ}$; chain distance $32 \mathrm{~mm}$; watermark identical with Sosower croix latine $45-46$.

Catchwords (scribal): horizontal, Ii3.

Quires: $6 \times 12$ (f. 105), $2 \times 1$ (f. 107).

\section{A and B.}

Binding: of white parchment over cardboard; flyleaves and pastedowns conjoint, unidentified watermark with escutcheon and the motto 'J ROIG FA DIA SERRA' [?], countermark 'F - S - MERCADE' [?].

Marks of ownership: (front pastedown) '24902', '59. F. 19 / 1246', 'Rosenthal 9|10' (front flyaleaf Ir) 'Hippocrates Aphorismi / Lez. XXIII 440335 / M ONJ', 'Aphorismi Hipocratis'.

Provenance: purchased by the Wellcome library in 1910 (accession number 24902).

Bibliography: Moorat, I.225-6; Duffy, op. cit. (note 84), 13, 18, 68-70; Touwaide, 539; Jouanna, op. cit. (note 48), lxxxv, xci.

Caroline Magdelaine, 'Le commentaire de Damascius aux Aphorismes d'Hippocrate', in Antonio Garzya and Jacques Jouanna (eds), Storia e ecdotica dei testi medici greci: Atti del II Convegno Internazionale, Parigi 24-26 maggio 1994 (Napoli: D’Auria, 1996), 289-306: 291, 293.

Sibylle Ihm, Clavis commentariorum der antiken medizinischen Texte (Leiden: Brill, 2001), 79-80, 203-4, 290.

Mark Sosower, Signa officinarum chartariarum in codicibus Graecis saeculo sexto decimo fabricatis in bibliothecis Hispaniae (Amsterdam: Haakert, 2004), 36, 276-7, 488.

86 Duffy, op. cit. (note 84). 


\section{MS.413}

Ottoman Empire, ca. 1800 (from watermarks).

Paper, $218 \times 158 \mathrm{~mm}$; ii + 14 + ii (foliated 1-14), linn. var., unruled.

[1r-8v] Ps.-Leo VI the Wise, Oracles. [10r-v] Arsenios Markellos, Oracles. [9r-v, 11r13v] Anonymous collection of oracles.

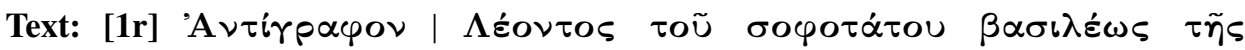

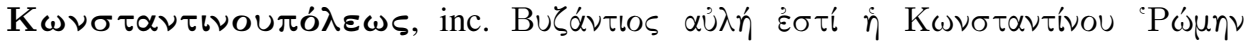

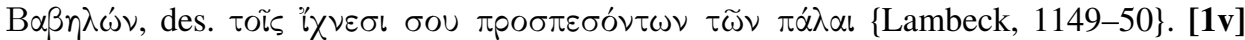

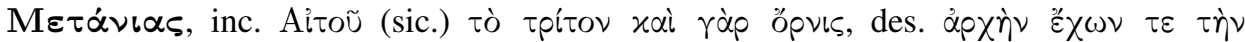

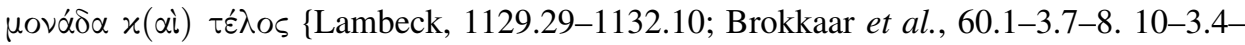

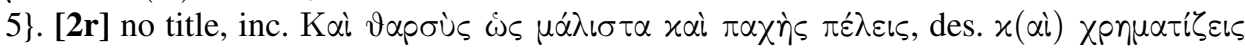

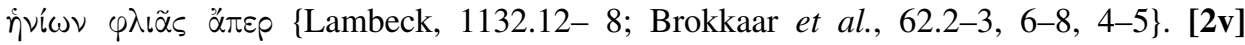

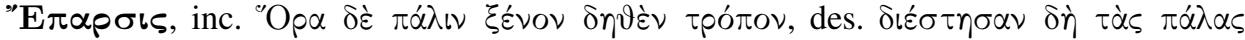

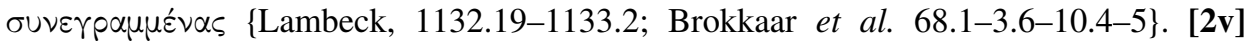

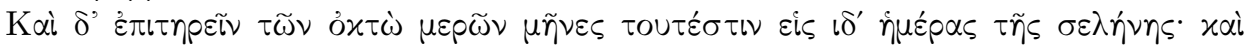

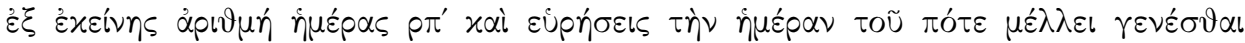

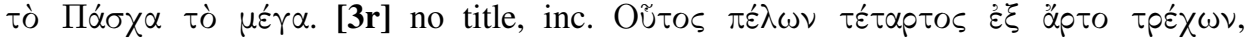

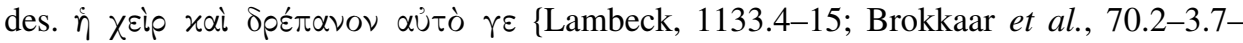

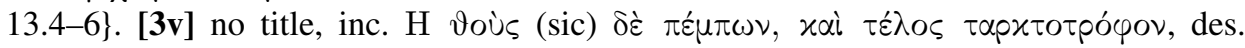

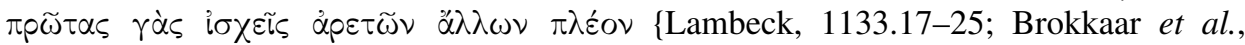

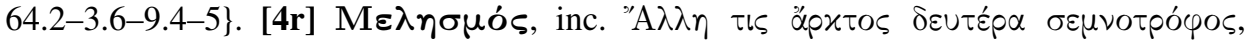

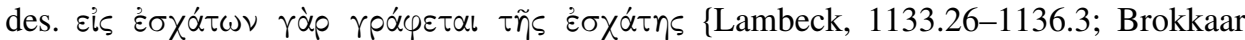

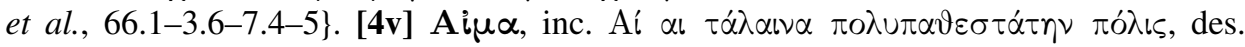

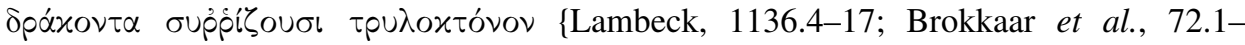

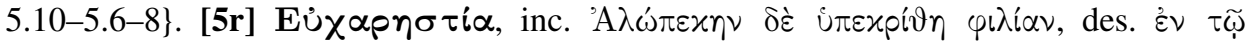

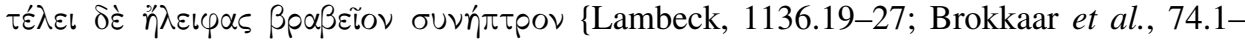

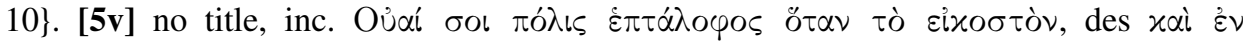

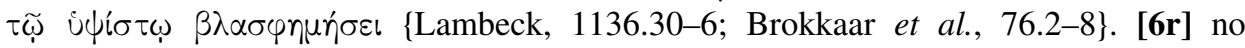

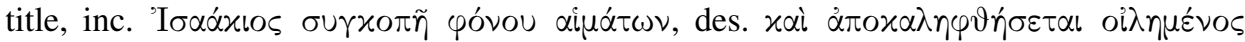

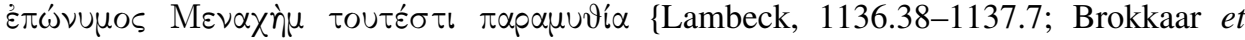

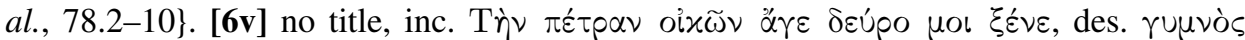

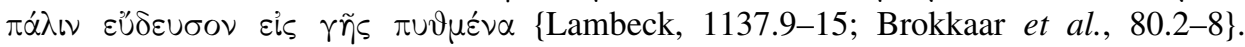

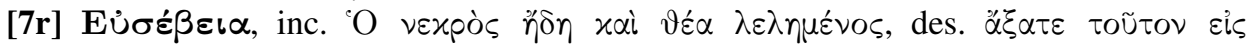

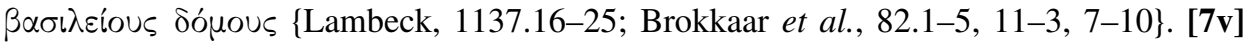

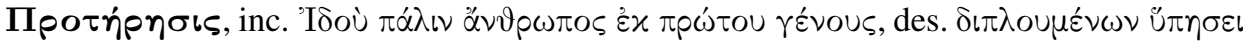

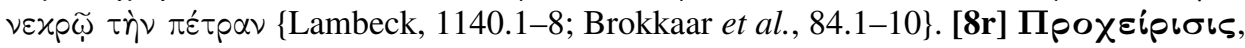

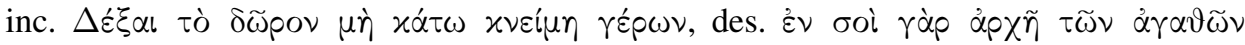

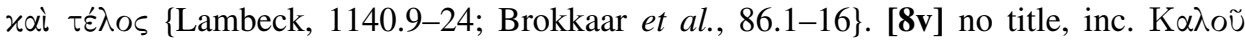

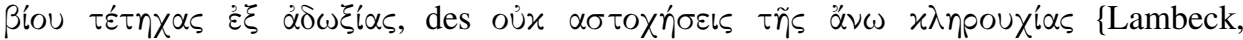

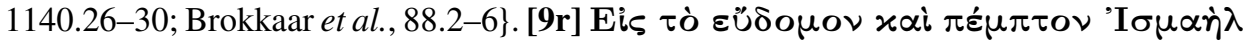

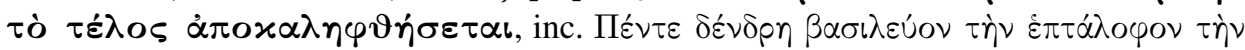

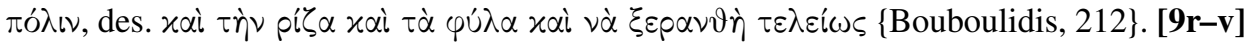

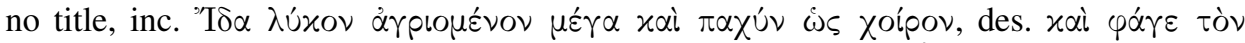

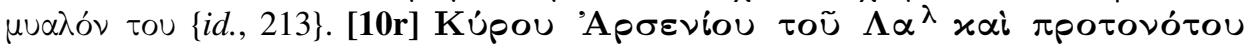

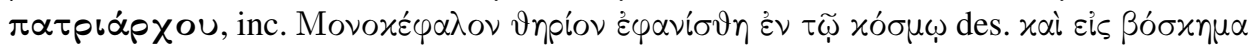

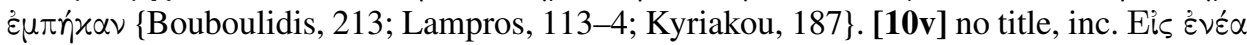




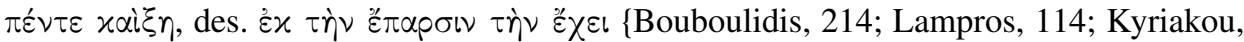

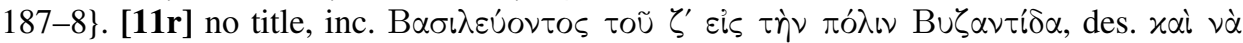

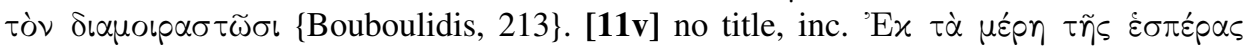

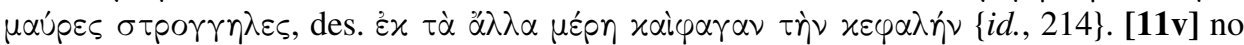

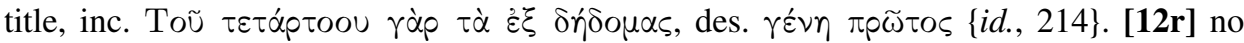

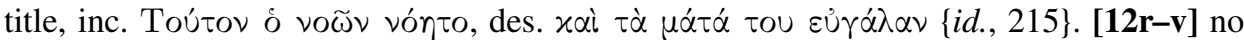

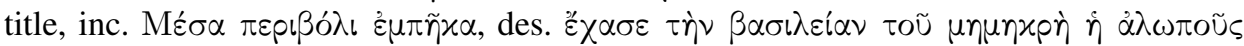

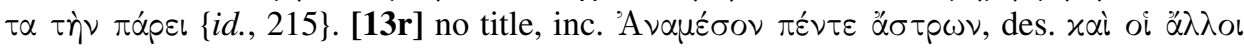

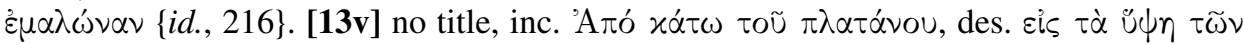

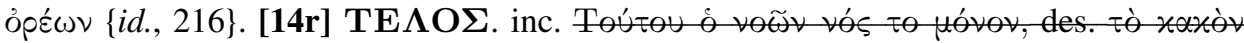
Onpiov

Handwriting: unidentified late post-Byzantine hand.

Illustrations: $(1 \mathrm{v}-11 \mathrm{v}, 12 \mathrm{v}-13 \mathrm{v})$ twenty-two figural drawings in ink and wash.

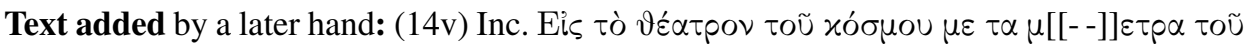

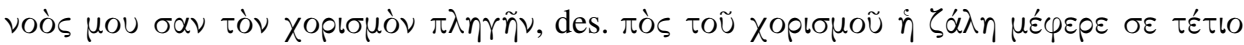

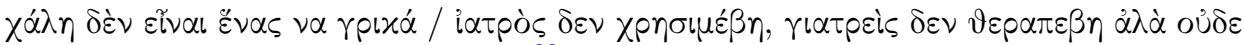
$\dot{\partial} \varphi \varepsilon \lambda \dot{\eta}\left\{\sim\right.$ Karatzas and Psalidas, 102\}. ${ }^{88}$

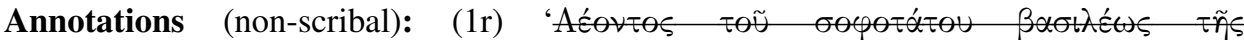

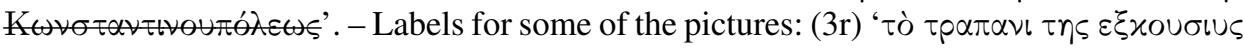

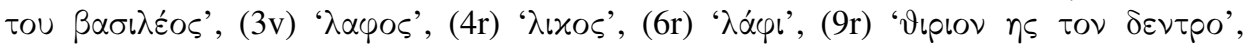

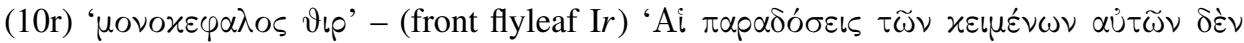

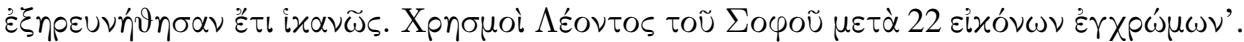

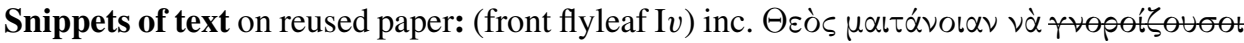

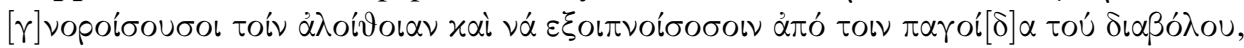

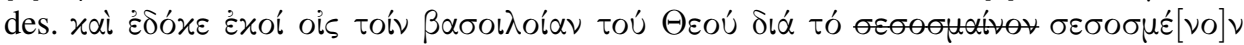

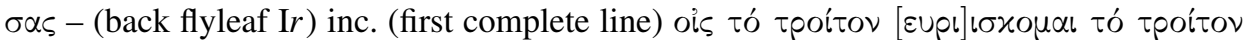

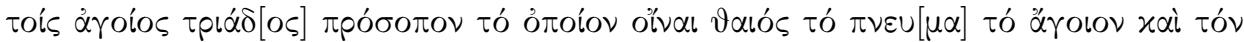

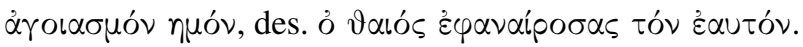

Paper: folded in $4^{\circ}$; chain distance $20 \mathrm{~mm}$; watermark generally similar to Eineder 315 (coat of arms with lion), attested in 1804; countermark with the letters FL under a decorated arch. ${ }^{89}$

Quires: 12 (ff. 1-11, 14) + 2 (ff. 12-13).

Binding: of marbled paper over cardboard; cloth pastedowns; the pastedowns of an older binding have been retained as front and back flyleaves.

\footnotetext{
87 Peter Lambeck (ed.), 'Imperatori Leonis cognomine Sapientis Oracula', in Jacques Paul Migne (ed.), Patrologiae cursus completus: Seria Graeca (Paris: Migne, 1863), Vol. 107, 1121-40; Walter G. Brokkaar et al., The Oracles of the Most Wise Emperor Leo \& The Tale of the True Emperor (Amsterdam: Leerstoelgroep Nieuwgriekse Taal en Letterkunde en Byzantinologie, 2002); Phaidon K. Bouboulidis, "I $\sigma \tau o p \eta \mu \varepsilon ́ v \alpha$

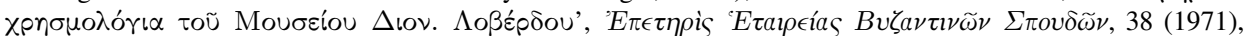

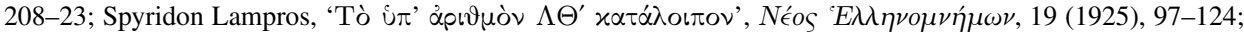

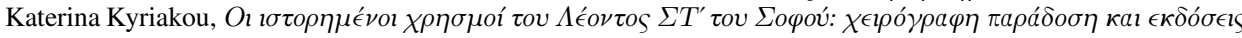

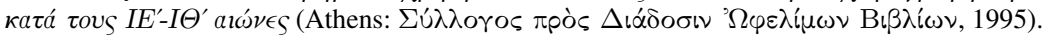

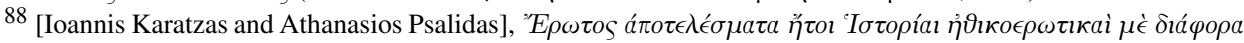

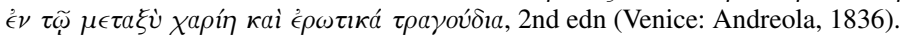

89 Georg Eineder, The Ancient Paper-Mills of the Former Austro-Hungarian Empire and Their Watermarks (Hilversum: Paper Publications Society, 1960).
} 
Marks of ownership: (front pastedown) label inscribed 'LH 8' - (1r) circular stamp 'GEORGE P. BEGLERY CONSTANTINOPLE' with 'Aplv. 79' handwritten in the centre.

Provenance: Georgios P. Vegleris (1850-1923), Constantinople (his no. 79) - Lionel Hauser (1868-1958), Paris (his no. 8); [his sale, Sotheby's, London, 17 April 1934, lot 331]; purchased on the behalf of Wellcome Library (accession number 66627).

Bibliography: Moorat, I.280-1.

Catalogue of the Very Extensive and Important Library of Early Books and Manuscripts Relating to Alchemy \& the Occult and Physical Sciences, the Property of M. Lionel Hauser (London: Sotheby \& Co, 1934), 36-7.

Lydie Hadermann-Misguich and Jeannine Vereecken, Les oracles de Léon le Sage illustrés par Georges Klontzas: la version Barozzi dans le Codex Bute (Venice: Institut Hellénique de Venise, 2000), 51.

\section{MS.498 (olim Nikolsburgensis II.241)}

Probably Constantinople, ${ }^{90} 1492$ AD (from paschal tables on ff. $66 \mathrm{v}-67 \mathrm{r}$ ).

Paper, $303 \times 212, \mathrm{i}+86$ (foliated $1-86) .{ }^{91}$

[23r-24r] Verses by Manuel Korinthios. [25r-27v] Anonymus, Epitome III of Hephaestion of Thebes' Apotelesmatika or Astrological Effects (TLG 2043.002). [31r] Ps.-George Chrysokokkes, List of Equivalent Ancient and Modern Toponyms. [31v] Anonymous verses. [32r-68r] Michael Chrysokokkes, Hexapterygon. [68v] Michael Psellos, Concise Answers to Various Questions, excerpt (TLG 2702.028).

Ruling (dry-point): (ff. 1-22, 28-30, 70-86) none; (f. 24) rectangular frame with squares inside [225 × 133]; (ff. 25-27) linn. 25-27, ca. [240 × 155], unclear ruling; (ff. 31-41) rectangular frames with lines inside, linn. 30-31 [225 × 138]; (ff. 42-69) ruling for tables, size varies from $[230 \times 165]$ to $[160 \times 115]$.

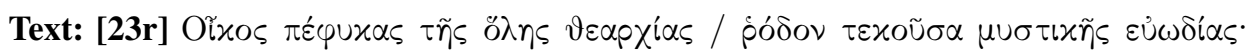

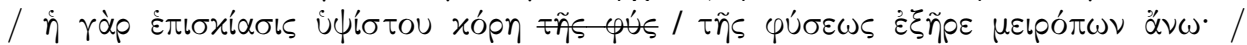

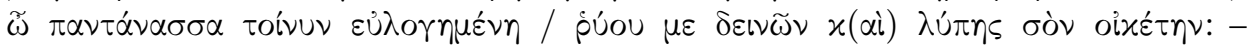

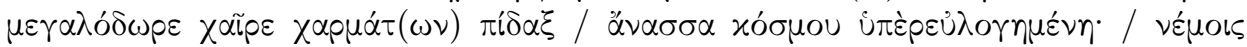

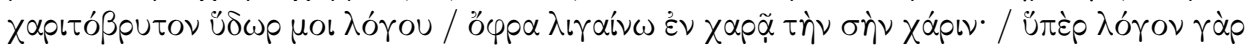

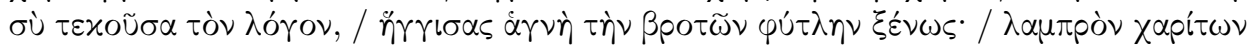

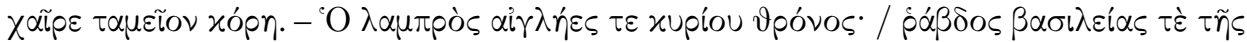

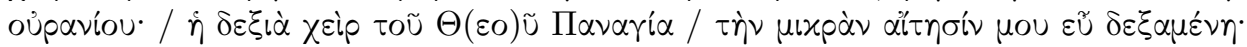

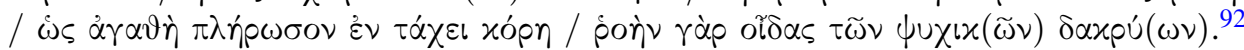

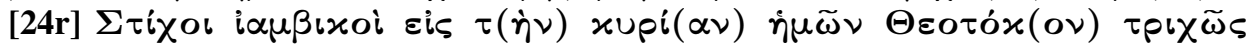

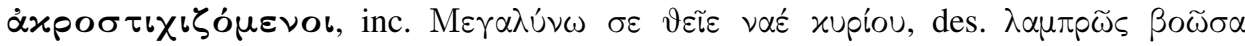

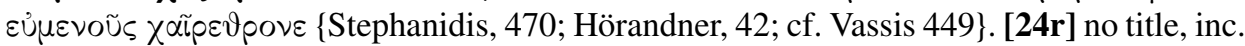

\footnotetext{
90 Based on Rudolf Stefec's identification of hand B with that of Manuel Korinthios, attested at the Greek Patriarchate of Constantinople in 1481-1507.

91 The current foliation is in the lower left-hand corner of each recto. An older foliation (1-87, incl. front flyleaf) is found in the upper left corners.

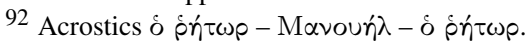




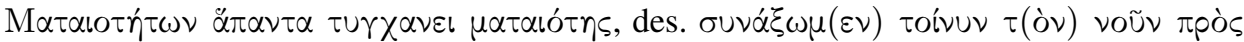

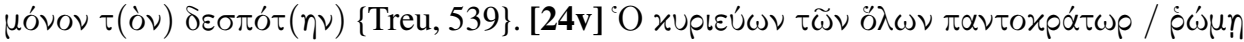

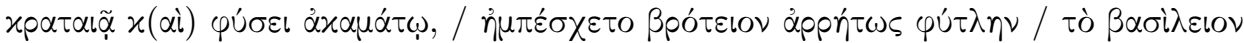

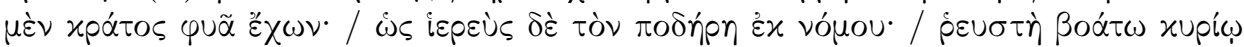

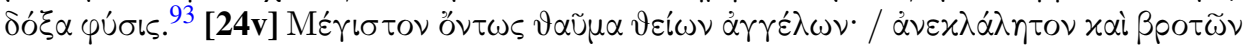

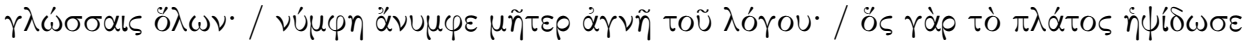

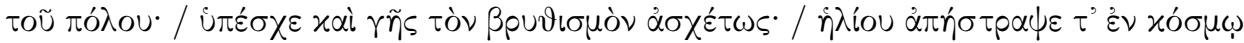

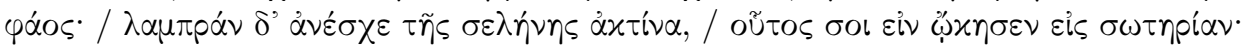

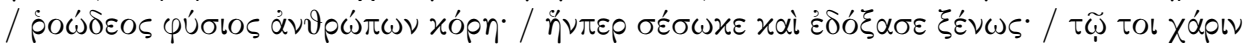

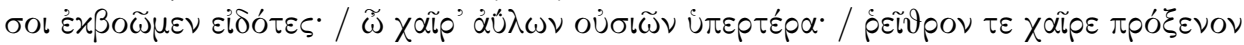

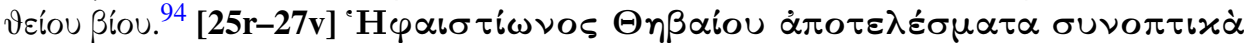

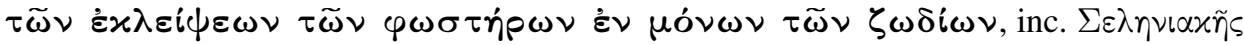

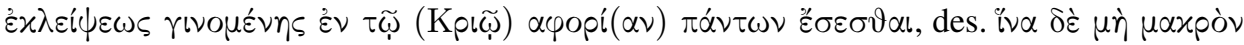

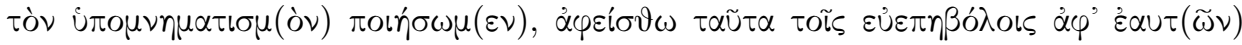

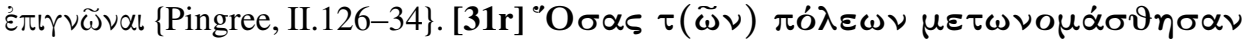

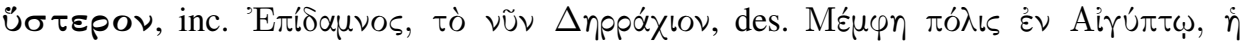

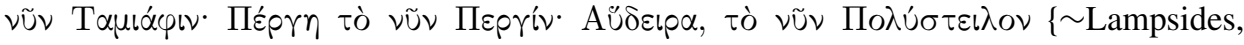

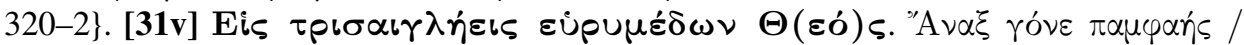

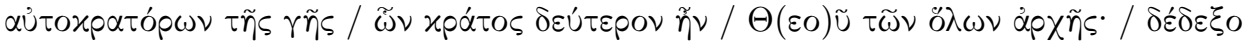

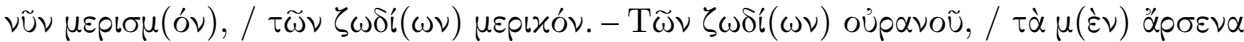

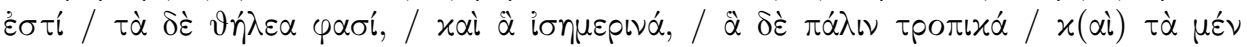

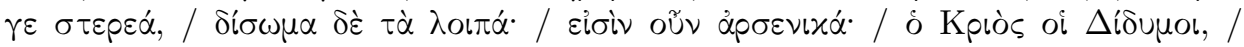

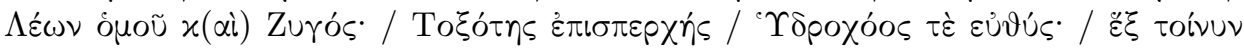

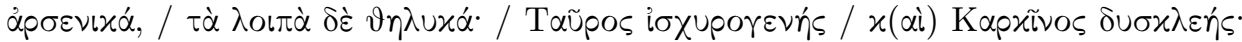

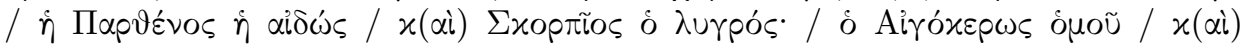

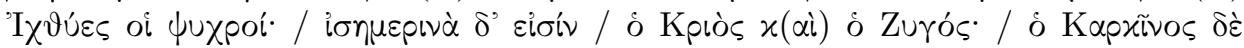

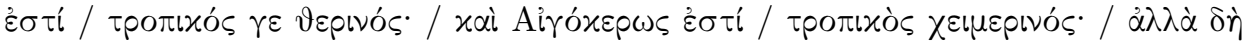

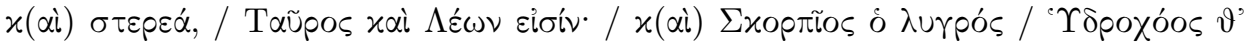

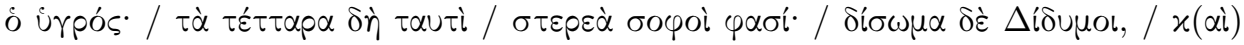

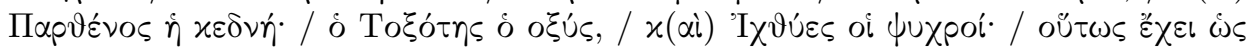

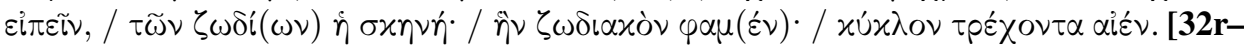

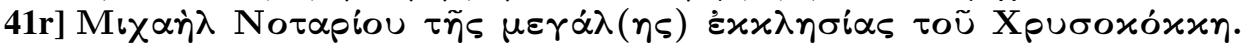

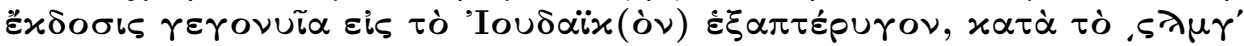

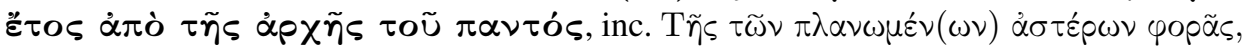

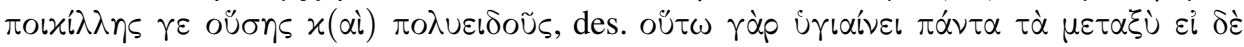

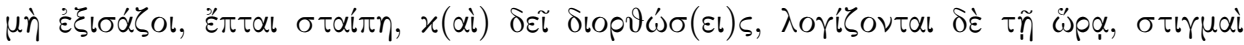

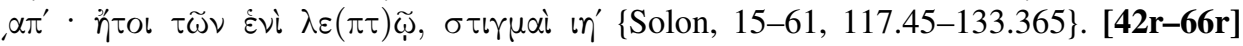

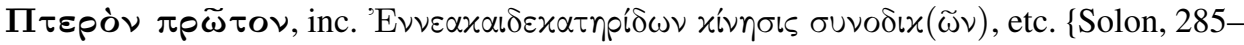

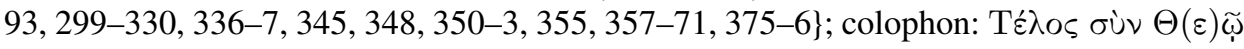

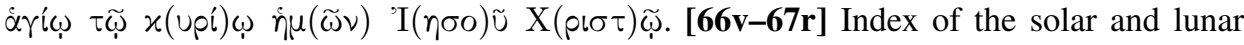
cycles and the moveable feast dates for the years $\zeta \alpha^{\prime}$ through $\zeta x \eta^{\prime}\{\sim$ Solon, 381-2\}. [67v] Index of the dominant zodiacal sign for each day of the twelve months \{ Solon,

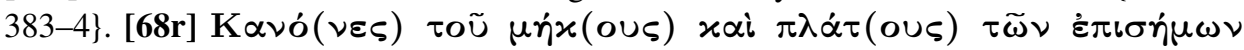

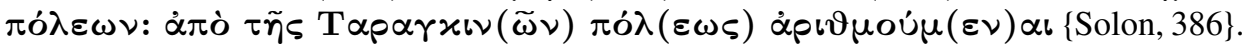

93 Acrostics ò ṕń $\tau \omega \rho$.

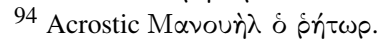




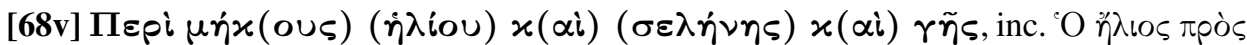

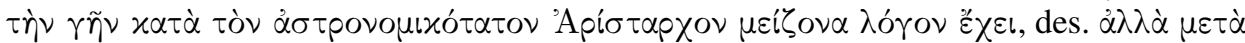

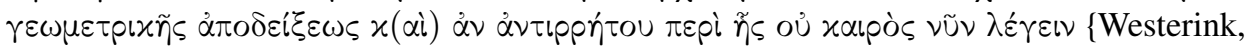
section $127,67-68) .{ }^{95}$

Blank pages: flyleaf $\mathrm{I} v$, ff. $1 \mathrm{r}-22 \mathrm{v}, 23 \mathrm{v}, 28 \mathrm{r}-30 \mathrm{v}, 41 \mathrm{v}, 69 \mathrm{r}-86 \mathrm{v}$.

Illustrations: $(59 \mathrm{v}-65 \mathrm{r})$ ink drawings of the zodiacal figures.

Handwriting: A (ff. 24r infra, 24v infra, 31r, 32r-41r), B (ff. 23r, 24r supra, 24v supra, $25 \mathrm{r}-27 \mathrm{v}, 43 \mathrm{r}-68 \mathrm{v})$. Rudolf Stefec identified scribe B with Manuel Korinthios. ${ }^{96}$

Annotations (non-scribal): (flyleaf I $r$ ) 'Titulus libri mathematici' - (flyleaf I $r$ )

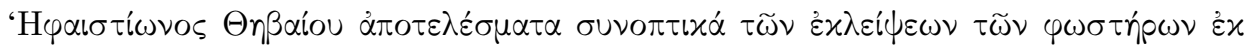
$\mu o ́ v \omega \nu \tau \widetilde{\omega} \nu \zeta \omega \delta i ́ \omega \nu$. Ebestionis Thebai opera consummata, compendiosa, de ecclipsibus luminarium coelestium, ex solis zodiaci circulis. (Secun)dus tractatus "O $\sigma \alpha \varsigma \tau \widetilde{\omega} \nu \pi \delta ́ \lambda \varepsilon \omega \nu$

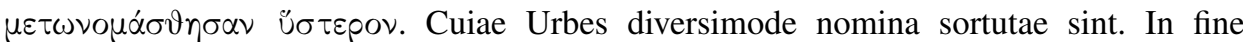
adiuncta sunt tabula Ecclipsium luminarium coelestium, iuxta doctrinam antecedentem.' - (back of side flap) arithmetical calculations.

Paper: folded in $2^{\circ}$; chain distance $36 \mathrm{~mm}$; watermarks very similar to Heawood 2467 (hand), attested in 1503, and to Piccard 155891 (hand), attested in 1522. ${ }^{97}$

Quires: 1 (flyleaf i), 4 (f. 4), 1 (f. 5, conjoint with front pastedown), 10 (f. 15), $2 \times 8$ (f. 31), $4 \times 10$ (f. 71), 8 (f. 79), 1 (f. 80, conjoint with pastedown), 6 (f. 86).

Binding original to the MS: Ottoman style, with a side flap; blind-tooled and gilttooled brown leather over cardboard. - Flyleaf i: chain distance $25 \mathrm{~mm}$; no watermark. - Pastedowns: same paper as the rest of the MS.

Marks of ownership: (front pastedown) engraved ex-libris inscribed: 'Ex Bibliotheca Illustris ac Generosi Domini D(omi)ni Ferdinandi Hoffman liberi baronis in Grunpühel et Strecau, D(omi)ni in Grevenstein et Ianowitz, supreme haereditarii curiae magistri ducatus Styriae et supremi marsalci archiducatus Austriae, sacratissmae: caesae: et regiae maiestatis consiliarii et camerae aulicae praefecti. etc. ${ }^{98}$

Provenance: Ferdinand Hoffmann (1540-1607), Freiherr von Grünbühel and Strechau; by descent $;{ }^{99}$ inherited by his great-granddaughters Maria Elisabeth Hoffmann (1663-1705)

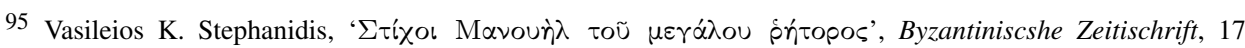
(1908), 470; Wolfram Hörandner, 'Visuelle Posie in Byzanz: Versuch einer Bestandsaufnahme', Jahrbuch der Österreichischen Byzantinistik, 40 (1990), 1-42; Vassis, op. cit. (note 52); Maximilian Treu, 'Manuel Holobolos', Byzantiniscshe Zeitischrift, 5 (1896), 538-59; David Pingree, Hephaestionis Thebani Apotelesmaticorum libri tres, 2 vols (Leipzig: Teubner, 1974); Odysseas Lampsides, 'Georges Chrysococcis, le médecin, et son oeuvre', Byzantiniscshe Zeitischrift, 38 (1938), 310-22; Peter Solon, 'The "Hexapterygon” of Michael Chrysokokkes' (unpublished PhD thesis: Brown University, 1968); Leendert Gerrit Westerink, Michael Psellus: De omnifaria doctrina (Nijmegen: Centrale Drukkerij, 1948).

96 On Manuel Korinthios, see Rudolf Stefec, 'Zwischen Urkundenpaläographie und Handschriftenforschung: Kopisten am Patriarchat von Konstantinopel im späten 15. und frühen 16. Jahrhundert', Rivista di studi bizantini e neoellenici, ser. II, 51 (2014), in press.

97 Heawood, op. cit. (note 14).

98 On this ex-libris, engraved by Lucas Kilian (1579-1637): Sibylle Appuhn-Radtke, 'Augsburger Buchillustration im 17. Jahrhundert', in: Helmut Gier and Johannes Janota (eds), Augsburger Buchdruck und Verlagswesen (Wiesbaden: Harrassowitz, 1997), 735-90: 758.

99 Klaus Eckart Ehrlicher, 'Ein steirisches Adelsgeschlecht in Böhmen und Mähren: Hoffmann Freiherren zu Gruenpüchel und Strechau', Bohemia: Zeitschrift für Geschichte und Kultur der böhmischen Länder, 21 (1980), $70-83$. 
and Johanna Maria Xaveria Hoffmann (d. 1706); presented to their guardian Ferdinand Joseph von Dietrichstein (1636-98) in $1679 ;{ }^{100}$ by descent; inherited by Hugo von Mensdorff-Pouilly (1858-1920), Fürst von Dietrichstein zu Nickolsburg (his no. II.241); inherited by Alexander Albert Olivier Anton von Mensdorff-Pouilly-Dietrichstein (18991964), Nickolsburg/Mikulov; [his sale, H. Gilhofer and H. Ranschburg, Lucerne, 25-26 June 1934, lot 352]; purchased on the behalf of Wellcome Library (accession number 66711).

Bibliography: Moorat, I.329-30; Pingree, op. cit. (note 95), Vol. 2, xix.

Eduard Gollob, 'Verzeichnis der griechischen Handshriften in Österreich ausserhalb Wiens', Sitzungsberichte der Kaiserlichen Akademie der Wissenschaften in Wien, Philosophisch-historische Classe, 146 (1903), 1-173: 89-90.

Bibliothek Fürst Dietrichstein (Luzern: Gilhofer \& Ranschburg, 1933-4), II.42 (no. 352).

Peter Solon, 'The Six Wings of Immanuel Bonfils and Michael Chrysokokkes', Centaurus, 15(1970), 1-20: 17.

\section{MS.4103}

Northern Greece, ${ }^{101} 1697$ AD (from the lunar tables on pp. 142-143).

Paper, $215 \times 150,125$ leaves (partially pagined: 55-76, 81-86, 89-100, 105-116, 119-164, 187-196, 209-212, 271-280, 277["]-324, 329-342, 379-387, 378 [ie. 388], [409]-412; then foliated: $1-24),{ }^{102}$ linn. $20-21$ [153 $\left.\times 92\right]-[160 \times 95], 00 D 1$.

Anonymous collection of post-Byzantine iatrosophia. ${ }^{103}$

Likeliest correct order of folia: 1, 3, 5, 23, 6-8, 15-20, 22, 5 (reversed), 24 (reversed), 91-4, 21

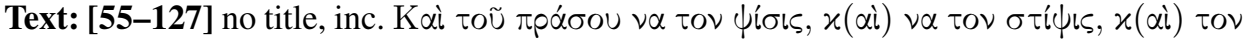

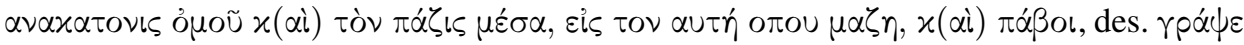

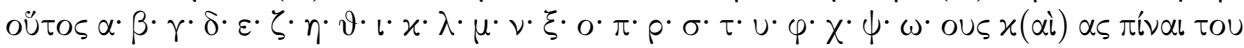

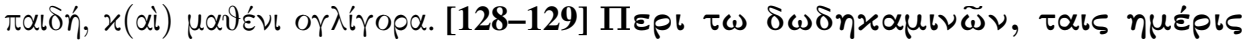

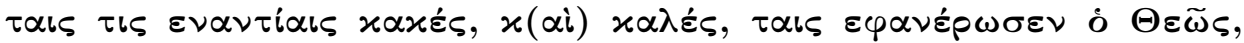

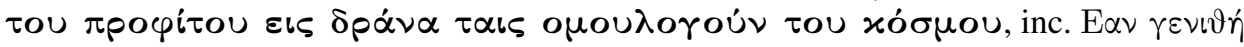

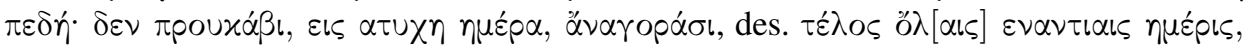

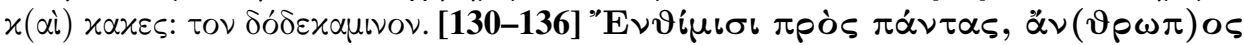

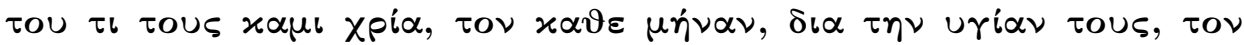

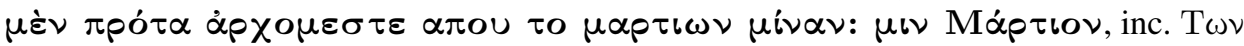

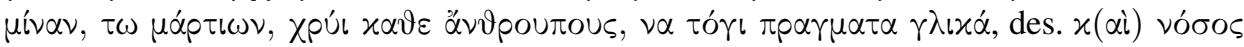

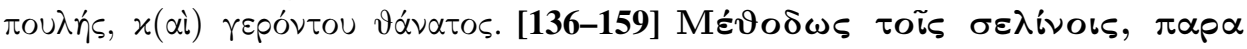

100 Paolo Divizia, 'Una lettera in lingua italiana di Ferdinand Hoffmann von Gruenpüchel und Strechau', Études Romanes de Brno, 37 (2007), 159-65: 160.

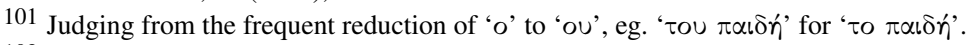

102 Many leaves are lost. There is no continuous modern foliation.

103 It contains instructions for diagnosis and phlebotomy, various recipes of composite drugs (generally structured a capite ad calcem), spells, short texts on auspicious days and on dream interpretation, moon-tables, and zodiacal tables. The text is not clearly organised and the original order of leaves remains uncertain. 


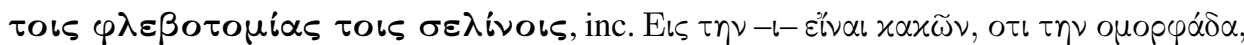

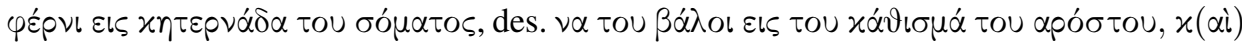

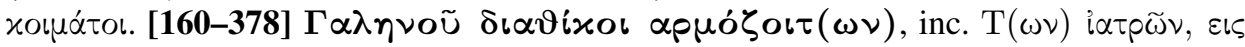

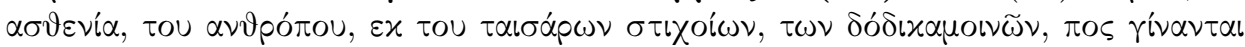

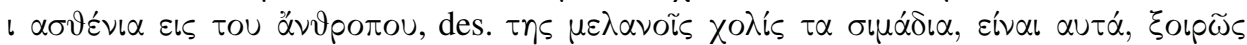

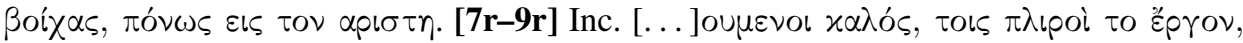

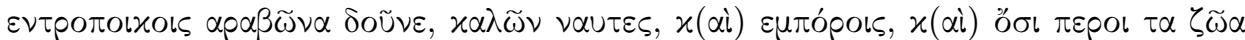

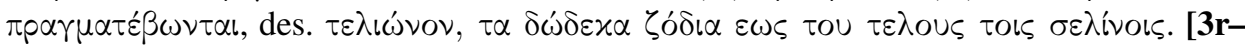

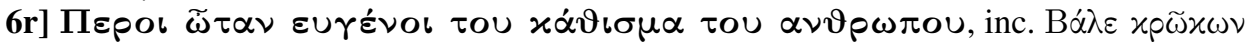

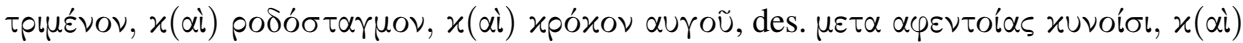

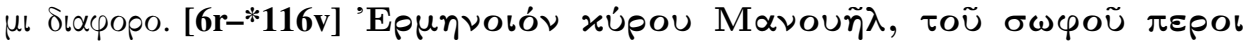

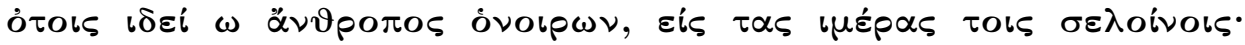

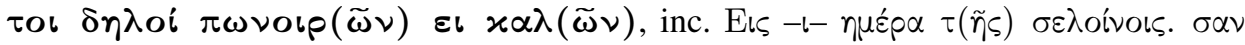

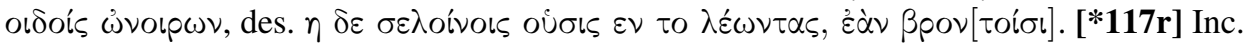

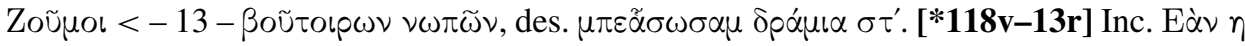

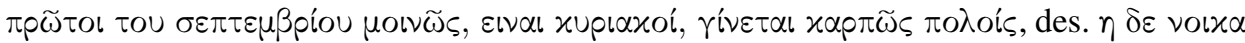

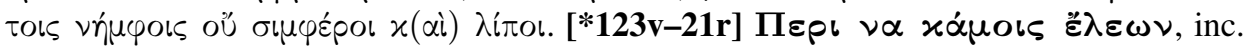

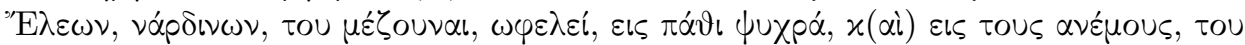

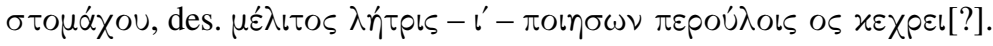

Annotations (scribal): (passim) occasional deletions/corrections to the text; $(* 31 \mathrm{v}, * 46 \mathrm{v})$ text in the outer margin - (pp. 280-281) additions to the main text.

Illustrations (scribal): (pp. 142-143) lunar tables for the years from 1697 to 1715 (p. 144, outer margin) drawings of a snake and a moon - (pp. 164, 290) apotropaic signs $(* 102 \mathrm{r}-\mathrm{v}, * 107 \mathrm{r}, * 118 \mathrm{r})$ zodiacal tables $-(* 117 \mathrm{v})$ table of the winds.

Handwriting: unidentified post-Byzantine hand.

Annotations (non-scribal): (pp. 96, 98, 135, 138, 139) marginal notes - $(* 125 \mathrm{v})$ poorly legible recipe.

Old pagination (possibly scribal): Greek numerals $S e, \lambda \gamma^{\prime}$ (p. 55) through $\nu \delta^{\prime}$ (p. 76), $\nu \vartheta^{\prime}$ (p. 81) through $\xi \delta^{\prime}$ (p. 86), $\xi \zeta^{\prime}$ (p. 89) through o $\eta^{\prime}$ (p. 100), $\pi \gamma^{\prime}$ (p. 105) through $4 \delta^{\prime}(116)$.

Paper: folded in $4^{\circ}$; chain distance $28 \mathrm{~mm}$; watermark very similar to Andreev 40.218 (attested in 1664 AD). ${ }^{104}$

Quire signatures (possibly scribal): Arabic numerals $I i 1,3$ (pp. 55, 57), 4 (p. 71), 7 (p. 119), 8 (p. 135), 9 (p. 151), 17 (p. 279[bis]), 18 (p. 293), 19 (p. 311)

Quires: 8 (p. 72), 6 (p. 86; innermost bifolium lost), 6 (p. 100; outermost bifolium lost), 6 (p. 116; outermost bifolium lost), $2 \times 8$ (p. 150), $1+6$ (p. 164; last leaf lost), 6 (p. 196; outermost bifolium lost), $2 \times 1$ (pp. 209-212), 6 (p. 280; outermost bifolium lost), $3 \times 1$ (p. 282[bis]), 6 (p. 292), 8 (p. 310), $1+6$ (p. 324; last leaf lost), $6+1$ (p. 342; first leaf lost), 1, 2, $3 \times 1$ (p. 3[8]8), 1 (p. 410), 2 (p. 411-f. 1); ff. 3, 5, 23, 6, 22, 5, 24 and 21 are singletons; 2 (ff. 7-8); 6 (ff. 15-20), 6 (ff. 9-14).

104 Stefan Andreev, Les filigranes dans les documents ottomans: couronne (Sofia: Trayanov, 2007). 
Binding: none.

Provenance: [sale, Sotheby's, London, 17 February 1936, lot 97]; purchased on the behalf of Wellcome Library (accession number 69261).

Bibliography: Moorat, II.877-8.

Catalogue of valuable printed books, illuminated and other manuscripts, autograph letters, topographical drawings of American interest, etc.: comprising, the library of Sir Algernon Methuen (...) on Monday, the 17th of February, 1936, and two following days at one o'clock precisely (London: Sotheby \& Co, 1936), 19. 